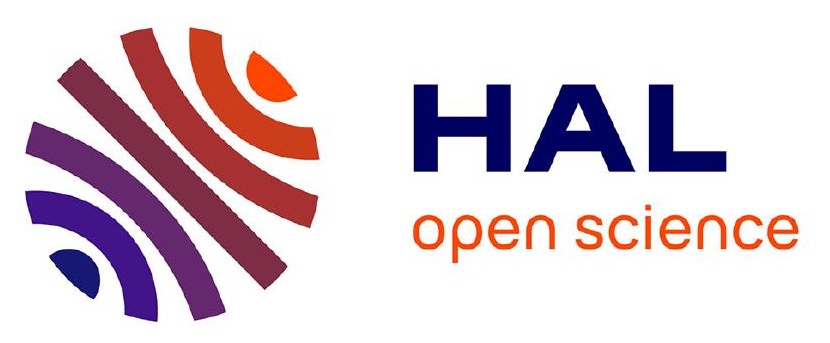

\title{
EthoLoop: automated closed-loop neuroethology in naturalistic environments
}

\author{
Ali Nourizonoz, Robert Zimmermann, Chun Lum Andy Ho, Sebastien Pellat, \\ Yannick Ormen, Clément Prévost-Solié, Gilles Reymond, Fabien Pifferi, \\ Fabienne Aujard, Anthony Herrel, et al.
}

\section{To cite this version:}

Ali Nourizonoz, Robert Zimmermann, Chun Lum Andy Ho, Sebastien Pellat, Yannick Ormen, et al.. EthoLoop: automated closed-loop neuroethology in naturalistic environments. Nature Methods, 2020, 17, pp.1052 - 1059. 10.1038/s41592-020-0961-2 . hal-02990284

\section{HAL Id: hal-02990284 \\ https://hal.science/hal-02990284}

Submitted on 17 Nov 2020

HAL is a multi-disciplinary open access archive for the deposit and dissemination of scientific research documents, whether they are published or not. The documents may come from teaching and research institutions in France or abroad, or from public or private research centers.
L'archive ouverte pluridisciplinaire HAL, est destinée au dépôt et à la diffusion de documents scientifiques de niveau recherche, publiés ou non, émanant des établissements d'enseignement et de recherche français ou étrangers, des laboratoires publics ou privés. 


\section{EthoLoop: Automated Closed-Loop Neuroethology in Naturalistic Environments}

Ali Nourizonoz ${ }^{1}$, Robert Zimmermann ${ }^{1}$, Chun Lum Andy Ho ${ }^{1}$, Sebastien Pellat ${ }^{1}$, Yannick Ormen ${ }^{1}$, Clément Prévost-Solié ${ }^{1}$, Gilles Reymond ${ }^{1}$, Fabien Pifferi ${ }^{2}$, Fabienne Aujard ${ }^{2}$, Anthony Herrel ${ }^{2}$, Daniel Huber ${ }^{1}$

1 University of Geneva, Department of Basic Neurosciences, Geneva, Switzerland

2 Musée National d'Histoire Naturelle, Adaptive Mechanisms and Evolution, UMR7179 - CNRS, Paris, France

\section{Abstract:}

Accurate tracking and analysis of animal behavior is crucial for modern systems neuroscience. Animals can be easily monitored in confined, well-lit spaces or virtual-reality setups. However, tracking freely moving behavior through naturalistic, three-dimensional (3D) environments remains a major challenge. A closed-loop control that provides behavior-triggered stimuli and thus structures a behavioral task, is also more complicated in free-range settings. Here, we present EthoLoop: a framework for studying the neuroethology of freely roaming animals, including examples with rodents and primates. Combining real-time optical tracking, "on the fly" behavioral analysis with remote-controlled stimulus-reward boxes, allows us to directly interact with free-ranging animals in their habitat. Assembled with off-the-shelf and wireless hardware, we show that this closed-loop optical tracking system can be used to follow the 3D spatial position of multiple subjects in real time, continuously provide close-up views, condition behavioral patterns detected online with deep learning methods and be synchronized with wirelessly acquired neuronal recordings or with optogenetic feedback. Reward or stimulus feedback is provided by battery-powered and remote-controlled boxes that communicate with the tracking system and can be distributed at multiple locations in the environment. The EthoLoop framework enables a new generation of interactive, but well-controlled and reproducible neuroethological studies in large-field naturalistic settings. 
A precise quantification of behaviour is essential for understanding brain function. Neuroscientists and ethologists have studied animal behaviour for decades seeking to identify the underlying neural circuits 1,2 . The quest for linking neuronal activity to specific behaviors in a laboratory setting imposes several constraints on the experimental framework ${ }^{3}$. Besides the obvious need for tractable variables, the studied behavior should retain most ethologically relevant sensory, motor, and cognitive components, while remaining reproducible. The environmental setting in which the animal moves should also be flexible enough to allow probing for multiple parameters involved in the studied behavior. Finally, access to simultaneous recordings or manipulations of neuronal activity is essential.

One strategy is to partially or fully immobilize the animals in a virtual reality (VR) environment ${ }^{4}$. This approach facilitates detailed observation of behavior and allows for accurate closed-loop stimulus control. It can also provide convenient and stable access to the brain for optical ${ }^{5,6}$ or electrophysiological recordings ${ }^{7}$. However, important drawbacks exist ${ }^{8}$. In most instances, natural motor output and the related sensory feedback are significantly disrupted; possibly inhibiting normal spatial processing $\left({ }^{9}\right.$, although see $\left.{ }^{10,11}\right)$. Furthermore, VR is often limited to a single sensory modality, such as vision ${ }^{4-6,12}$, somatosensation ${ }^{13}$ or olfaction ${ }^{14}$. Finally, VR systems are inherently poorly suited to study motor patterns such as climbing or jumping and probe social interactions involving multiple animals.

An alternative approach is to retain the animal's full sensory-motor experience by studying its freely moving behavior in naturalistic conditions. This however poses challenges regarding behavioral control and quantification. It necessitates knowing where the animal is and what it does, as well as tracking the identity of multiple subjects. Systems for tracking in naturalistic environments have been developed for several animal species, including insects ${ }^{15-19}$, rodents ${ }^{20-22}$, primates ${ }^{23,24}$ or flying bats ${ }^{25,26}$. Multiple subject identification can be achieved based on the recognition of coat color ${ }^{27}$, wearable colored markers ${ }^{23,28}$, electronic markers ${ }^{29}$ or bayesian estimation ${ }^{17}$. Finally, immersive visual VR for unrestrained animals has also been developed ${ }^{11,30,31}$. However, by projecting a single viewpoint into the arena, these systems are currently restricted to the use with single animals, and provide only non-binocular views. In general, most tracking systems are limited by small and relatively empty laboratory environments, and require bright and even illumination (although see ${ }^{23-25}$ ) to extract position, behavior or to provide feedback. Studying animals in more naturalistic environments with noisy or changing backgrounds and poorly lit conditions (e.g. in arboreal and nocturnal settings), poses serious challenges to most existing tracking systems.

Finally, interacting with freely roaming and fast moving animals in a closed-loop manner for behavioral conditioning, would require a tracking framework to be extremely fast, robust and provide sufficient high resolution information about the tracked animal. However, most current systems for freely moving animals are still designed for offline behavioral analysis (although see ${ }^{11,19,30-33}$ ) and can thus not react to behavioral elements in the order of tens of milliseconds. Taken together, there is currently a real need for an integrated framework able to carry out the next generation of reproducible neuroethological experiments with freely moving animals in more naturalistic settings. 


\section{Results}

\section{Implementation of the EthoLoop tracking system}

To follow the behavior of freely roaming nocturnal animals, such as mice (Mus Musculus) or mouse lemurs (Microcebus Murinus), a small arboreal primate, in naturalistic 3D environments, we developed an ultra-fast, multi-camera closed-loop tracking system capable of providing simultaneous close-up views and analyses of the ethology of tracked individuals (Fig. 1a). We hence termed the system EthoLoop.

The identification and tracking of individuals in the dark is either achieved using retro-reflective passive markers (Fig. 1b, Methods, Supplementary Video 1) or, when multiple individuals have to be tracked simultaneously, by spectrally separating the hue of miniature battery-powered infrared Light Emitting Diodes (LEDs) with different wavelengths (Fig. 1c, Supplementary Fig. 2, Supplementary Video 2). To increase the speed and decrease the processing time, the spatial localisation and spectral separation of each camera view in the EthoLoop system is carried out on stand-alone graphical processing units (GPUs (Fig. 1a, Methods). The position of each animal is then transformed into real World 3D coordinates by geometric triangulation from the different camera views (Fig. 1a, ${ }^{17,23}$, Methods). We achieved tracking at rates ranging from $580 \mathrm{~Hz}$ to $785 \mathrm{~Hz}$ (for 1 to 3 targets, Fig. 1f), with delays below 8 ms (up to 3 targets, Fig. 1g). The ideal number of cameras (minimum 2) depends on the complexity of the obstructing structures in the environment. Adding more cameras also improves the tracking accuracy in larger arenas. Thus, by combining off-the-shelf hardware and open source code, this system can be easily adapted to spatial scales of $1 \mathrm{~m}^{3}$ (Fig. 1a, Supplementary Video 1), $10 \mathrm{~m}^{3}$ (Fig. 1c, Supplementary Videos 2-3) and up to $>100 \mathrm{~m}^{3}$ (Supplementary Fig. 1, Supplementary Video 4). Tracking multiple animals in such large scale arenas while preserving their respective identity allows detailed study of their social interactions (Supplementary Fig. 3). Finally, to facilitate the installation of additional cameras in large-scale and challenging conditions (trees, branches), all cameras and GPUs can be battery-powered and communicate via wireless network protocols (Methods), thus removing the need for external wiring and facilitating their placement in strategic locations.

Although the GPU-based 3D tracking allows determining the animal's position with high temporal resolution (Fig. 1f-g) and precision (Supplementary Fig. 1d), the detailed nature of the behavior remains unknown. Inspired by the pioneering work on freely flying and walking insects ${ }^{15,17}$, we therefore added a gimbal-mounted, close-up video camera to the EthoLoop system, providing a focused and magnified view continuously centered on the tracked individual (Fig. 1c, 1e, Supplementary Video 3). This way, the behavior of a tracked animal can be analyzed in detail, independent of the actual size of the tracked volume in which it navigates. Since jumping primates can reach speeds up to $2 \mathrm{~m} / \mathrm{s}$ ( Supplementary Video 3), the focusing mechanism of the close-up system was driven by an ultrafast liquid lens (Methods). To ensure sufficient illumination of the tracked subject in large scale environments, an infrared light source was mounted in parallel to the camera path (Supplementary Video 4) or on a separate gimbal system (Methods). This allowed a targeted and economic illumination independently of any ambient light. In summary, the EthoLoop system combines ultrafast and accurate tracking the position of animals even in large scale, arboreal or nocturnal settings with high-resolution close-up views of their behaviors. 

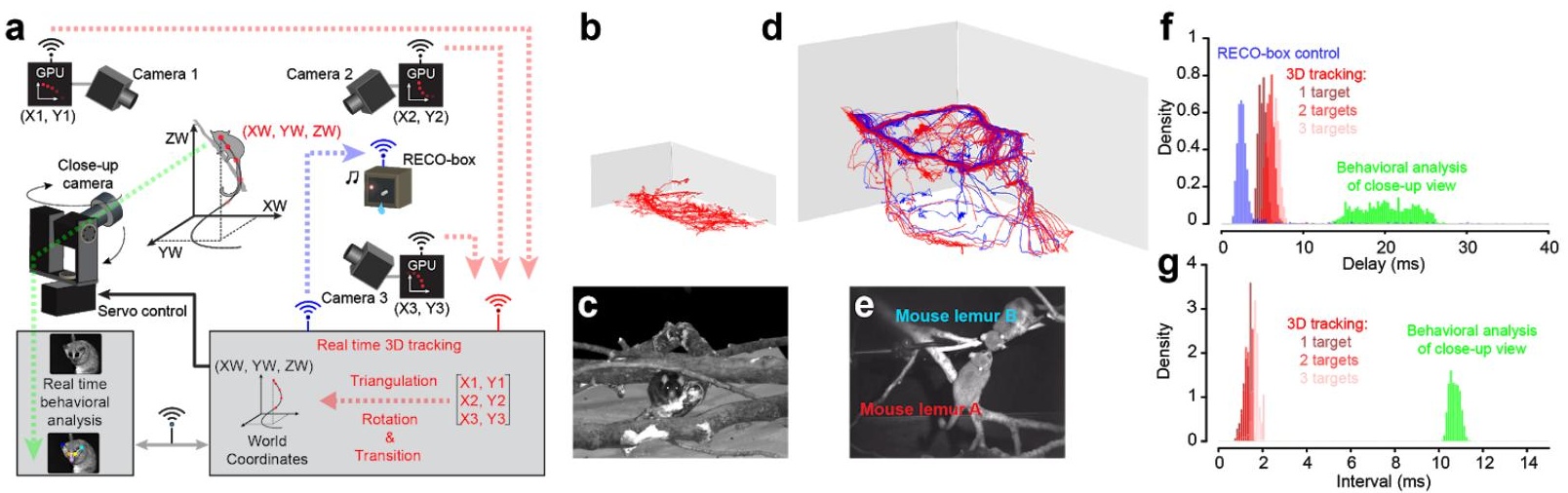

Figure 1: The EthoLoop behavioral tracking system. a) Schematic depiction and spatial arrangement of different EthoLoop elements. Multiple infrared cameras (Camera 1-3) with dedicated GPUs process images from different viewing angles. The identity and positions of the detected markers are wirelessly transmitted to a central host computer for 3D reconstruction (triangulation, followed by rotation and transition into real World 3D coordinates). The 3D coordinates are forwarded to control the angles and autofocus of a gimbal-mounted close-up camera. The images from the close-up system are either saved for offline analysis or processed "on-the-fly" to trigger remote-controlled reward boxes (RECO-box). RECO boxes can provide auditory or visual stimuli or liquid rewards and are connected wirelessly (wifi-symbols). b) 3D trajectory of a mouse tracked in a naturalistic nocturnal environment (red line, $60 \mathrm{~min}$, arena $0.9 \times 1.8 \times 0.5 \mathrm{~m}$, see Supplementary Video 1), covered with wooden branches. The tracking was based on a passive retro-reflective marker fixed around the neck. c) Still image from the infrared close-up camera following the position of the tracked mouse in b. d) Thirty minutes of 3D trajectories of two mouse lemurs (Mouse lemur A in red, Mouse lemur B in blue) tracked in a $10 \mathrm{~m}^{3}$ arena filled with tree branches. e) Infrared close-up image of the two tracked mouse lemurs (from d). f) Histogram of EthoLoop latencies for the communication with the RECO-box (blue), reconstructing 3D position (1-3 targets, dark to light red), and the real-time behavioural analysis (green). g) Histogram of subsequent 3D reconstitution time (1-3 targets, red) and time for the analysis of subsequent close-up images.

\section{Observation and manipulation of natural foraging behavior using remote controlled reward sources}

Out of all animal behaviors, foraging is most likely one of the most universal activities ${ }^{34,35}$. It was probably a prominent driver behind major evolutionary specializations of sensory, motor and cognitive capacities in primates and other clades ${ }^{36}$. To study naturalistic foraging in laboratory settings, the distribution of food sources, as well as sensory cues signaling their availability would ideally be controlled by the experimenter in real time, taking into account the animal's actual position and behavior. This way, specific situations could be created in a reproducible manner. We therefore integrated small, remote-controlled and interactive reward boxes (RECO-box) into the EthoLoop framework. The RECO-boxes are able to play sensory cues (auditory or visual) and provide calibrated amounts of liquid rewards (Fig. 1a, 3, Methods). Multiple of these battery-powered boxes can be distributed in the environment to mimic the availability and cues associated with natural resources.

To illustrate the range of basic behavioral patterns in freely roaming mouse lemurs, we positioned multiple RECO-boxes in the behavioral arena (Fig. 2a, Supplementary Fig. 4). To cover the volumetric space more evenly and make the experiments more reproducible, we also replaced the naturalistic branches with a parameterizable artificial lattice maze ${ }^{37,38}$ (Methods). The availability of a liquid reward at a given location was indicated by an auditory cue $(9 \mathrm{kHz}$ tone, 1 s intermittent at $0.5 \mathrm{~Hz}$ ). To mimic a variable degree of predictability of the next food location, the different boxes were first activated sequentially in a fixed order (Fig. 2b \& c, Supplementary Video 5, Supplementary Fig. 4b \& c). This type of activation triggered highly stereotypical and most likely memory guided paths (Fig. $\mathbf{2 b} \& \mathbf{c}$, Supplementary Fig. $4 \mathbf{b} \& \mathbf{c}$ ). On the other hand, the random activation of a RECO-box (triggered by the specific spatial location of the animal, Fig. $\mathbf{2 d}$, Methods) established a situation where the animal could 
not predict the next reward location, and hence resulted in more variable behavioral patterns (Fig. 2d). The location-triggered random activation allowed us to have the mouse lemurs cover a large part of the 3D lattice maze (Fig. $\mathbf{2 b}$ \& c). These data illustrate how combining real time tracking with remotely controlled elements can rapidly shape specific behaviors and force the animals to adopt different foraging strategies.

Other, more global environmental parameters such as illumination also affect behavior in a given environment ${ }^{35}$. To highlight how our system can detect behavioral signs related to such global parameter changes, we reduced the illumination levels from $\operatorname{dim}(<1$ lux, Fig. 2e) to complete darkness (Fig. 2f, Supplementary Video 6). This triggered a profound change in the movement parameters including a significant reduction in the number of jumps (Fig. $\mathbf{2 g}$, Wilcoxon signed rank test, $P=0.0207$, $\mathrm{N}=12$ time intervals), or the locomotor speed distribution (Fig. 2h, Supplementary Video 6, Two-sample Kolmogorov-Smirnov test, $P<0.0001$ ).

Taken together, the combination of the EthoLoop tracking system with interactive agents such as RECO-boxes or changes of global parameters like illumination, seems ideally suited for exploring different aspects of ethologically relevant parameters within naturalistic settings.

a
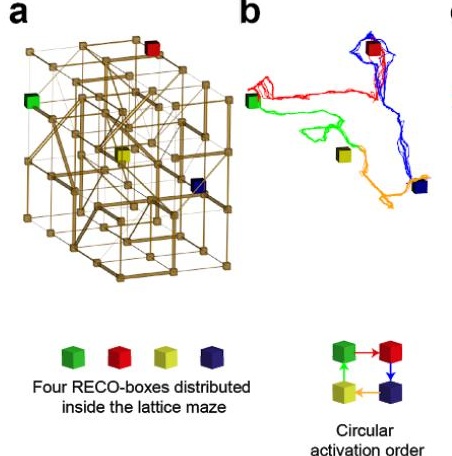

c
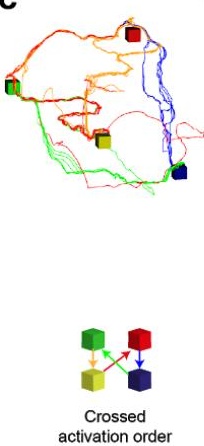

d

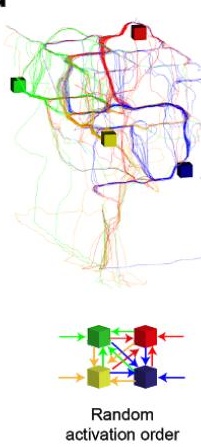

e

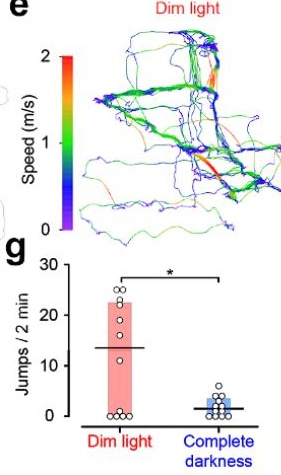

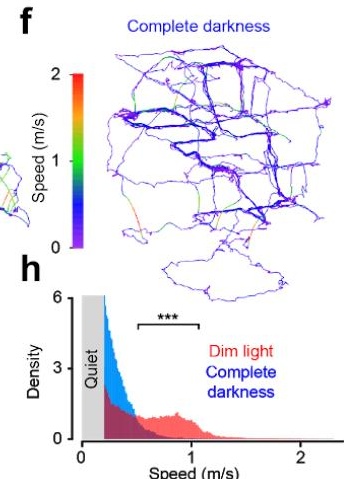

Figure 2: Foraging in free ranging mouse lemurs. a) Four RECO-boxes (colored cubes) spatially distributed at different locations of a lattice maze $(0.5 \mathrm{~m}$ branch length). RECO-boxes are shown in different colors and are activated with different sequences. Reward availability was indicated by a $9 \mathrm{KHz}$ tone. The actual reward delivery was made conditional upon the animal's arrival at the feeder within $10 \mathrm{~s}$. b) Ten minutes of 3D trajectory of the mouse lemur where the RECO-boxes are activated in a circular or crossed order (c). The trajectory color indicates the currently activated RECO-box. d) 3D trajectory of a mouse lemur when activation order was random and conditional on the actual position of the animal in the arena. e) Color coded running speed under conditions with dim light and complete darkness in (f). The number of jumps (g) as well as the overall speed (h) was reduced in the absence of light (blue) compared to the dimly lit condition (red). The ends of the boxes in ( $g$ ) represent the upper and lower quartiles and the middle lines represent the median.

\section{Fully automated conditioning of behavior in naturalistic environments}

To explore the possibility of using the EthoLoop system to reinforce specific behaviors by operant conditioning ${ }^{39}$, we combined the principle of "clicker training" by human experts ${ }^{40,41}$ with real-time and automated behavioral analysis. An auditory bridging stimulus ( $9 \mathrm{Khz}$ tone) followed by a small liquid reward from one of the RECO-boxes was triggered upon an automated detection of a specific behavior. To test the effectiveness of such automated conditioning procedures, we first set out to condition mice to enter one of four corners of a rectangular arena (Fig. 3a). Upon detecting an entry into a defined corner, the tracking system triggered a sound from a single RECO-box and a reward was available for the 
subsequent 10 seconds in the center of the arena. Mice rapidly $(<30 \mathrm{~min}$ ) learned the task (Fig. $\mathbf{3 b}$ - $\mathbf{d})$ and the number of entries into the conditioned area significantly increased, compared to the initial rate and to the previously conditioned place ( $N=6$ mice; two way ANOVA test; interaction of two main effects $\mathrm{P}<0.0001$; Tukey post hoc test; ${ }^{* * *} P<0.0001$, Supplementary Fig. 5-6 for all days of training of all mice). Similarly, we were able to condition freely roaming mouse lemurs to climb repeatedly to an experimenter-defined position within the 3D lattice maze (Fig. 3e, Supplementary Fig. 7). The number of entries increased over a 30 min session (Fig. 3f-h, Supplementary Fig. 7).

a
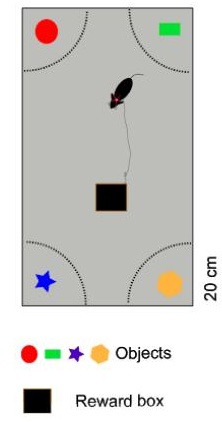

e

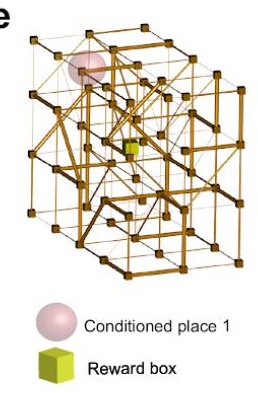

b

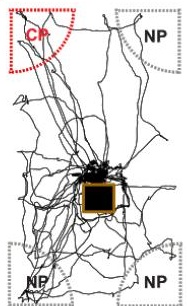

C

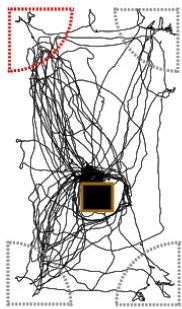

Middle

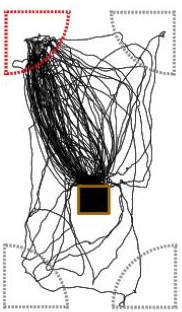

End

d

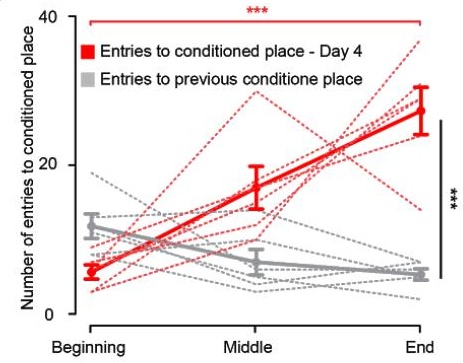

h

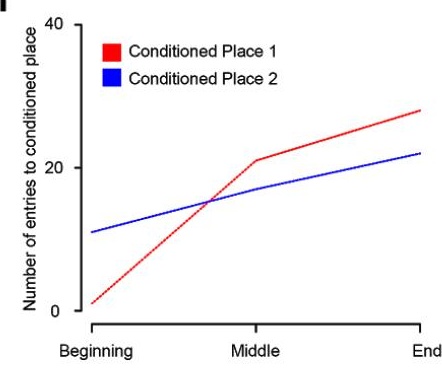

Figure 3: Fully automated place conditioning. a) A platform for freely moving mice containing a single RECO-box and differently shaped objects placed at the four corners. The mice were trained to visit one of the four corners of the platform. The reinforced corner was changed daily. b) Example traces of a mouse in the beginning, middle and end of a behavioral session. c) Number of entries to the conditioned place across a behavioral session. d) Number of entries to the conditioned place (red) compared to the entries to the non-conditioned corners (grey, $\mathrm{N}=6$ mice). Mice entered the conditioned place more frequently while the entrance to the previously conditioned place decreases over the three time intervals. Bars represent standard error of mean. e) Place conditioning with mouse lemurs in a 3D lattice maze. The blue sphere indicates the conditioned space. f) Example traces in the 3D maze of a mouse lemur in the beginning, middle and end of a behavioral session. c) Number of entries to the conditioned place across a behavioral session. $\mathbf{h}$ ) The number of entries increases for different conditioned places. The two colors represent the conditioning at two different locations in the arena ( $\mathrm{N}=1$ mouse lemur, see the additional trajectories in Supplementary Fig. 5).

Inspired by more sophisticated behavioral conditioning experiments carried out by professional animal trainers with pets or zoo animals ${ }^{40,41}$, we further sought to use the real-time close-up video stream to automatically detect specific motor patterns ${ }^{42}$ and reinforce them using the RECO-boxes. As a proof of principle, we chose to reinforce rearing behavior, which is observed spontaneously in both mice and mouse lemurs. To detect rearing, we first trained a deep learning network (DeepLabCut ${ }^{43}$ ) to track different body parts in a set of hand-annotated close-up movies (Fig. 4a, Methods). In a second step, we adapted DeepLabCut to classify the streamed images and localize the position of the different body parts in real-time in the stream of close-up images (Fig. 4a). Using this procedure we were able to identify body parts at an average rate of $93 \mathrm{~Hz}$ (Fig. 1f, green histogram) using a standard commercial 
graphics card (Methods). In a third step, the rearing behavior was detected using a set of geometrical rules (Methods) taking into account the relative spatial position of body parts. Reinforcing automatically detected rearing with a "click" ( $9 \mathrm{kHz}$, see Methods), followed by a reward from a nearby RECO-box (Fig. 5a), mouse lemurs rapidly increased the number of rearings (red arrows in Fig. 4c, Fig. 4d-e, Supplementary Video 7). In parallel, we also conditioned mice to rear on top of a platform in a flat arena (Fig. 4e, N=3 animals). They learned the task within 30 minutes (Fig. 4f-h, Supplementary Video 8). In this case, rearing was classified by using the $z$ axis of the marker position (Fig. 4f). These experiments illustrate how real-time and automated behavioral classification by the EthoLoop system can be efficiently used to shape or reinforce specific actions in situations which would have been very challenging for any trained human observer (due to fast moving subjects, poor illumination, large arenas or partial occlusions).
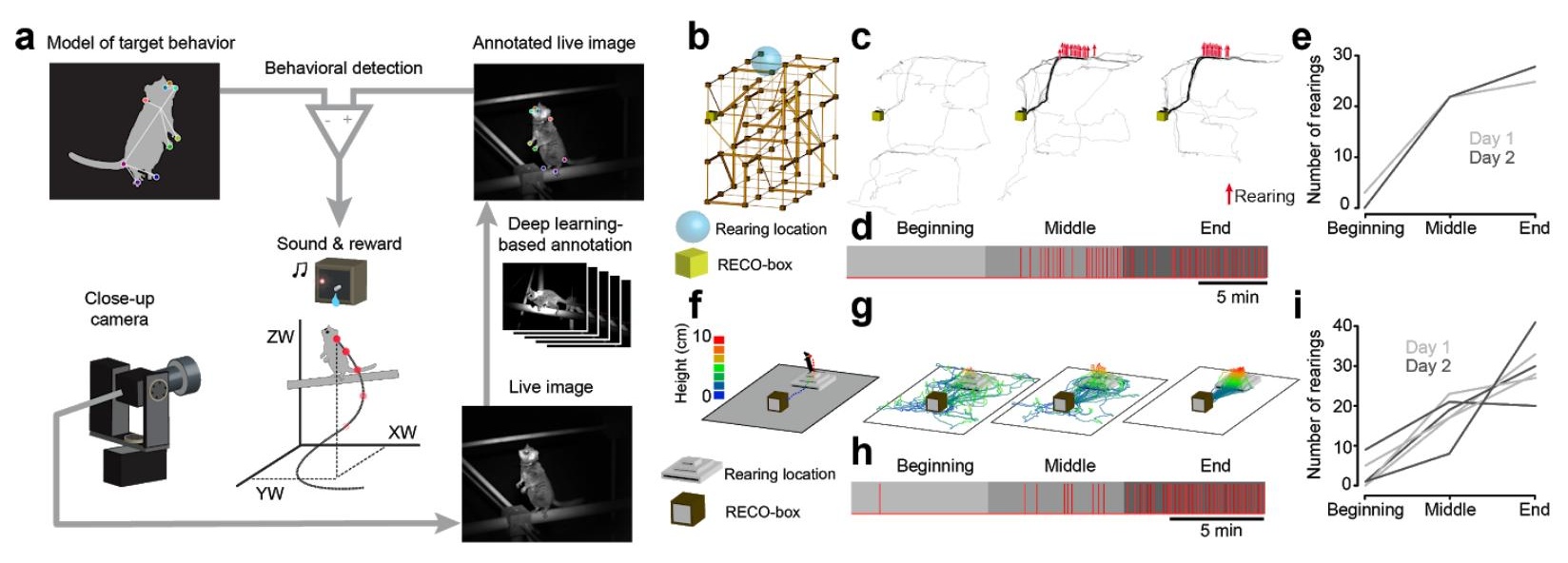

Figure 4: Operant conditioning of automatically detected behavior. a) Schematic representation of the information flow. While the close-up camera provides high-resolution live images of the tracked animal, the body parts are classified in real time by the weights of a pre-trained deep learning network (deep learning based annotation). A match of the annotated live image with a geometric model of the behavior to be reinforced, will trigger a sound, followed by a reward in the RECO-box. b) The lattice maze with a RECO-box (yellow cube) and an experimenter-defined area where rearing was conditioned (blue sphere). c) Three-dimensional trajectory of a mouse lemur during a conditioning session. The red arrows indicate rearing. d) The number of rearings detected across the session in c). e) The number of rearings increases within a behavioral session. f) Mouse arena with an elevated platform and a RECO-box. The color code indicates the height of the mouse. g) Example trajectory of a mouse during condition session where the number of rearings increased over the session (h). i) Summary data from 3 mice across two days ( $\mathrm{N}=3$ mice).

\section{Operant conditioning by optogenetic stimulation of reward circuits}

Using behavior triggered operant conditioning with actual rewards can become problematic when multiple subjects are present in the vicinity, since the reinforcement should target only one individual. Individualized conditioning can be achieved by optogenetic tools, such as the selective activation of dopamine (DA) neurons in the ventral tegmental area (VTA ${ }^{44}$ ). We tested whether behaviors detected by the EthoLoop system can also be reinforced by real-time optogenetic stimulation of VTA DA neurons in mice. We first performed place conditioning in an open field arena. Upon entry at a predefined location, mice received a brief stimulation (burst of $8 \times 4$ ms at $30 \mathrm{~Hz}$ ) of VTA DA neurons through a fiber optic cannula connected to a blue laser. The observed place preference spatial maps reveal that the mice were successfully conditioned (Fig. 5b, Supplementary Fig. 8). In a second set of experiments, we stimulated VTA DA neurons upon the detection of a predefined behavioral syllable ${ }^{42}$. As previously (Fig. 4), we chose to reinforce rearing on the hind limbs. Upon reinforcement with optogenetic VTA stimulation, mice started rearing significantly more within the specified area (Fig. 5c \& 5d, 
Supplementary Fig. 9, Supplementary Video 9). These experiments show that the EthoLoop system is well suited to provide precisely timed and behaviorally triggered optogenetic stimuli to freely moving animals in a fully automated manner and thus shape specific behavioral elements within tens of minutes.
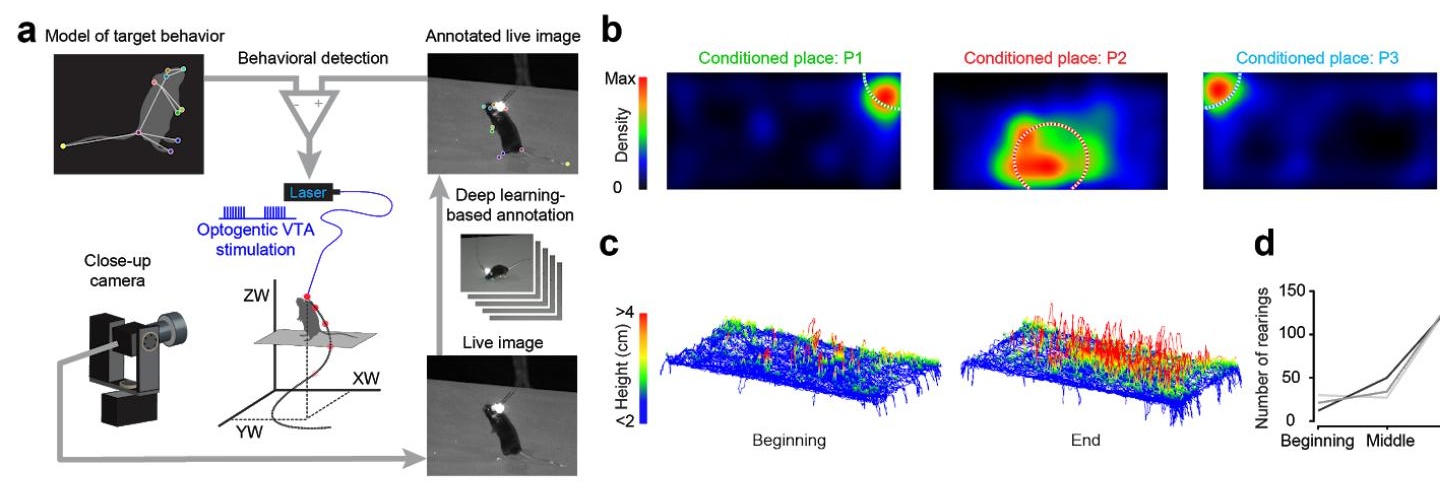

C

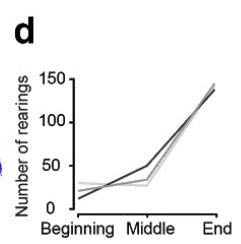

Figure 5: Reinforcing automatically detected behaviors with optogenetic VTA stimulation. a) Schematic representation of the information flow during optogenetic VTA DA neurons stimulation. The steps are identical to Fig. 5, except that the detection of a behavioral event triggers an optogenetic stimulation of VTA DA neurons. b) Heat map (2D kernel density estimation) after optogenetic place conditioning in an open field (size $0.8 \times 1.6 \mathrm{~m}$ ). The dotted areas indicate the boundary where the optogenetic stimulus was activated. The maps represent the probability density of the location of the mouse during the last third of conditioning session. The reinforced location was changed once the mouse spent in total more than 6 minutes inside the conditioned place. c) 3D trajectory of a mouse at the first and last 20 minutes of a optogenetic reinforcement of an automatically detected behavior syllable (rearing). The color code indicates the height of the mouse. d) Number of rearings detected at the beginning, middle and end of the behavioral session ( $\mathrm{N}=3$ mice).

\section{Combining 3D tracking with wireless neuronal recordings}

The ultimate goal of many neuroethological studies is to link a specific behavior to its underlying neuronal activity. To illustrate the feasibility of simultaneous neuronal recordings in combination with actively controlled foraging (Fig. 2), we set up a miniature wireless electrophysiological recording system for freely moving mouse lemurs (Methods). We recorded neurons in the dorsal CA1 of hippocampus (Supplementary Fig. 10), an area related to various aspects of spatial navigation ${ }^{45-47}$.

By synchronizing the recorded activity with the 3D position of the mouse lemur (Fig. 6, Methods) we found neurons that were responsive to a large region (floor, Fig.6a-b; Two-sample Kolmogorov-Smirnov test; $P<0.0001$ ) or a specific location (single branch, Fig. $6 c-d$, Supplementary Fig. 11) of the lattice maze, or only when moving in one direction on a given branch diameter (Fig. 6e-j; Kruskal-Wallis test; Wilcoxon rank sum test; $\left.{ }^{* * *} P<0.001 ;{ }^{* *} P<0.01 ;{ }^{*} P<0.05\right)$. Importantly, the EthoLoop system allowed us to induce the specific foraging patterns and thus ensure that the animals either cover large parts of the lattice maze (Fig. $6 \mathbf{a} \&$ c) or create multiple reproducible paths across a given location (Fig. 6e). These recordings are to our knowledge among the first examples of 3D place-cell like activity recorded in freely roaming primates and illustrate the vast potential for future experiments when combining the EthoLoop system with wireless electrophysiology. 

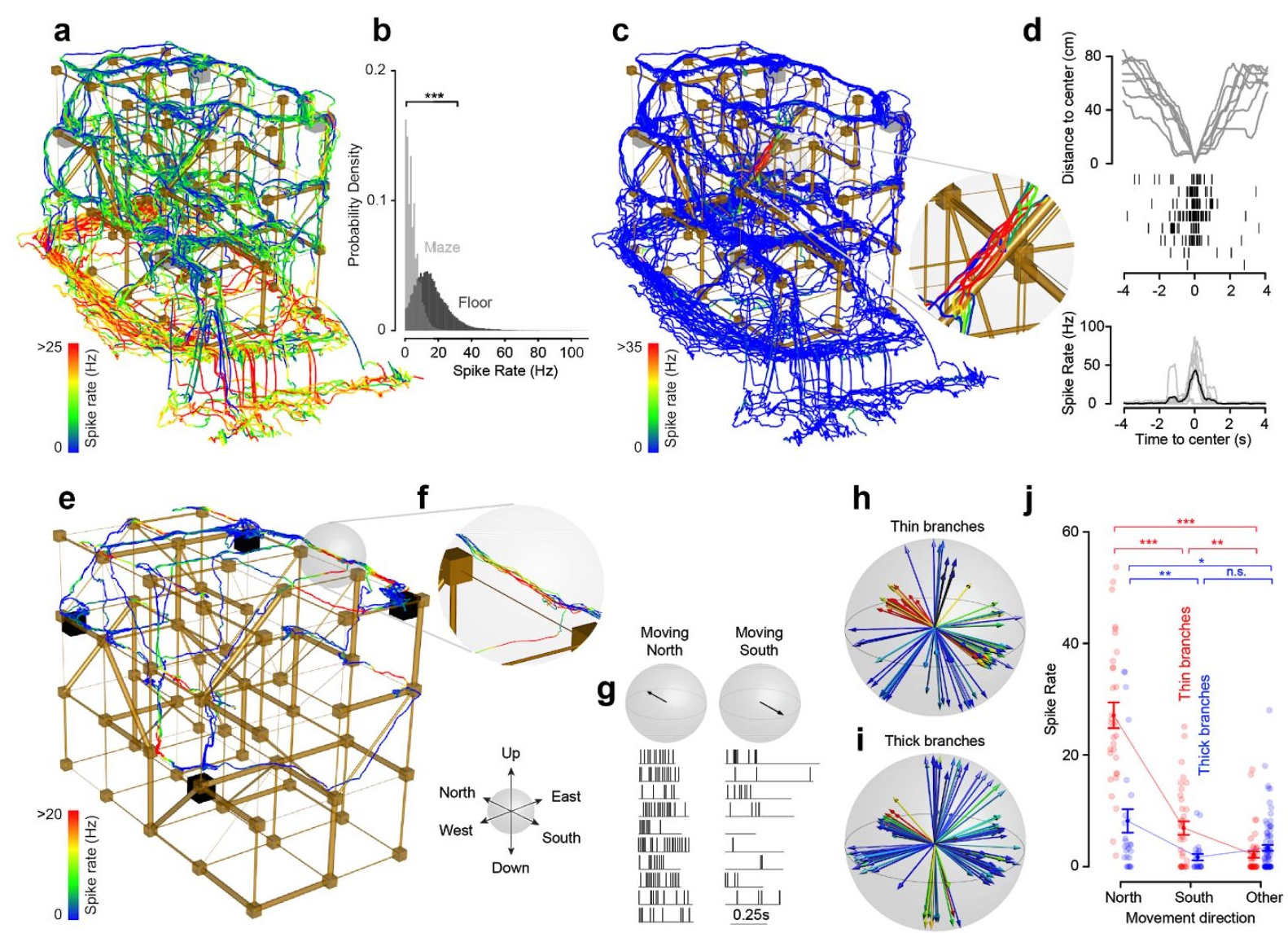

Figure 6: Wireless recordings of neuronal activity in dorsal CA1 during navigation in a 3D lattice maze. a) Example neuron showing increased activity when the mouse lemur was exploring the floor of the behavioral arena. b) The neuronal activity over $13 \mathrm{~cm}$ of displacements on the floor compared to the activity in the rest of the lattice maze. c) Example neuron showing increased activity preferentially on a single branch location (same recording session as in a). d) Top: Distance of the animal to the location with highest average activity on the branch. Middle: Action potentials of the recorded neuron which increase only when the animal passes this location. Bottom: Spike rate of the recorded neuron during individual passages (grey) and their average (black). e-f) Example neuron showing increased activity on multiple locations in the maze. g) Additional analysis revealed that the neuronal activity was specific to one specific spatial direction (north, neuronal data from the location in the gray sphere in e)). $\mathbf{h}$-j). Additional analysis revealed that the activity was higher when moving north on thin (top) compared to thick branches (bottom). The arrows in the sphere indicate the movement directions $(13 \mathrm{~cm}$ segments), while the color indicates the level of activity. j) This neuron showed the highest activity when moving on a thin branch towards the north of the maze. Bars in all panels represent the standard error of mean.

\section{Discussion}

EthoLoop is a video tracking framework that allows following animal behavior in naturalistic environments (Fig. 1). More importantly, it can actively shape behaviors via closed-loop interactions (Fig. 2-6). Such active interaction with the tracked subjects opens possibilities for many types of experiments ranging from automated reinforcement of innate sequences to the learning of complex cognitive tasks.

Furthermore, its scalability from a $1 \mathrm{~m}^{3}$ (Fig. 1b, 3, 5) to $\sim 10 \mathrm{~m}^{3}$ (Fig. 1, 2, 3, 4, 6 and Supplementary Fig. 3) and up to $100 \mathrm{~m}^{3}$ arena (Supplementary Fig. 1c), as well as its wireless connectivity, make it a powerful tool for characterizing complex behaviors in large scale and naturalistic settings. The possibility 
of using batteries as a power source for cameras and RECO-boxes (Methods) furthermore opens the door towards future applications to study the behavior of wild animals in their natural habitats. Adding more tracking and close-up cameras will be necessary, however, if foliage or branches are too obstructive or if the behavior of multiple animals needs to be characterized simultaneously with high resolution.

The overall principles of the EthoLoop framework (close up view, online classification, interaction with RECO-boxes) are also applicable to other real-time tracking systems ${ }^{11,15-18,23,29}$ and might thus be easily combined with already existing hardware (as long as the relative position can be updated fast enough). Finally, the recent emergence of novel markerless methods for tracking multiple subjects ${ }^{19}$ or for behavioral analysis ${ }^{43,48}$ can also be combined with this framework.

Since location, timing and type of activated cues and rewards can be arbitrarily controlled, the EthoLoop system is ideally suited to study behaviors such as foraging ${ }^{34}$. Foraging provides a unique setting where the limits of multi-sensory integration, sensory-guided motor control and high level cognition can be probed in a reproducible manner. Furthermore, foraging often requires that rules are learned rapidly, often in single trials. Combining foraging with well-studied cognitive behavioral paradigms such as attentional set shifting (for an example in the mouse lemur see ${ }^{49}$ ), might allow for more rapid learning and thereby avoid stereotypical or inflexible behaviors associated with overtraining. Finally, foraging is not only ideally suited to probe the neuronal circuits related to 3D spatial navigation ${ }^{25,26,38,50}$, but it might also become a powerful tool for dissecting neuronal dynamics underlying decision making and economic choice ${ }^{34,51}$.

We have shown that synchronized optogenetic manipulations and electrophysiological recordings during free navigation are in principle feasible. Similar to the pioneering work with freely flying bats ${ }^{25,26,50}$ we have chosen data loggers as recording devices for the neuronal signals. This necessitates that the neuronal data is aligned post-hoc with the spatial and behavioral data. For future closed-loop experiments involving triggering events by neuronal activity, actively transmitting recording systems (i.e. ${ }^{52}$ ) will be necessary.

Finally, this study highlights how closed-loop control of multiple stimulus and reward devices can rapidly shape the behavior and thus become a tool for systems neuroscience research. Technically, using wireless sensors and actuators and automated analysis using machine-learning in neuroethological research can be considered a particular extension of the "Internet of Things" (IoT, ${ }^{53}$. Hence, new hardware and software development from the loT field will most likely inspire the design of improved devices and enable better behavioral paradigms and analysis methods, thereby opening the door to novel applications for studying more complex behaviors.

Acknowledgments: We would like to express our gratitude to Martine Perret and the animal caretakers at the Brunoy facility for their help with mouse lemur breeding, care and handling; $\mathrm{H}$. Clamouze for constructing the maze. The members of the Huber lab for their support and discussions. We would like to thank Mackenzie and Alex Mathis, as well as Michael Scharter for their advice with implementing DeepLabCut. We would like to thank Pierre Yger for his advice on spike sorting, Lofti Slimani and Nicolas Liaudet for their help with the in vivo reconstructing of the electrode position. We would like to thank 
Mario Prsa, Gregorio Galinanes and Michael Long for their comments on the manuscript. This work was supported by the Vahabzadeh Foundation (ANZ), Human Frontiers Science Program (DH and FP, RGP0024/2016), the New York Stem Cell Foundation (DH). DH is a New York Stem Cell Foundation-Robertson Investigator.

\section{Author contribution}

ANZ and DH conceptualized the EthoLoop system. ANZ and DH designed the tracking and close-up hardware. ANZ wrote all software for acquisition, tracking, close-loop control and RECO-boxes. ANZ ran all experiments. ANZ analyzed all the data. DH oversaw data analysis. ANZ, DH and SP designed the RECO-box. RZ carried out the surgeries and ran the electrophysiological recording experiments. AH reconstructed the electrode trajectories. CPS provided the mice and advice for the optogenetic experiments. YO participated in the limb tracking. FP and FA provided expertise, guidance and resources related to the mouse lemurs. AH provided the 3D lattice maze and advice regarding the CT scan. GR provided expertise regarding the tracking software. DH and ANZ wrote the manuscript. 


\section{Methods}

\section{Hardware}

\section{Infrared real-time video tracking system}

Four to six high-speed USB3 cameras (MQ013CG-ON, XIMEA) were used for tracking. The cameras were equipped with wide-angle lenses (NAVITAR, f4.5 mm, 1.4) and infrared long-pass filters (FGL 665, Thorlabs, or $832 \mathrm{~nm}$ Bandpass Filter, Edmund Optics) to eliminate light in the visible range. Each camera was connected to an independent Graphical Processing Unit (GPU) based microprocessor (Jetson TK1 or TX2, NVIDIA, running Linux4Tegra) via a USB connection. All microprocessors were connected via ethernet (TK1) or WIFI (TX2) to a central host machine (Optiplex 9020 - Intel Core i7, DELL, running Linux Ubuntu 16.04 LTS) via a wireless router (RT-AC88U, ASUS). An additional USB3 camera (MQCG-ON, XIMEA) was connected to the host machine for permanent monitoring of the experimental room. Cameras and GPU units could either be powered by wall power supplies or with $12 \mathrm{~V}$ car batteries (44 Ah, Miocar).

\section{Portable active markers}

To track multiple animals simultaneously, infrared (IR) LED light sources of different wavelengths were used (SMC660, SMC750, SMC830, Roithner). These wavelengths are beyond the visible spectrum of mouse lemurs, and therefore do not interfere with their photoperiod ${ }^{54}$. To make the light spot visible from wide angles, the light was diffused using $6 \mathrm{~mm}$ opal glass diffuser (\#46163, Edmund Optics). To hold the light sources on the top of the neck of the mouse lemurs, the animal carried a custom collar around its neck with a miniature rechargeable battery attached below (Lipo 3.7 V, 140 mAh, LP451430). The total weight of the neck collar including battery was less than $4 \mathrm{~g}$.

\section{Portable passive markers}

Passive reflective markers consisted either of small, commercially available retro-reflective spheres (14 $\mathrm{mm}$, Optitrack), or custom made with flexible retroreflective transfer foil (Seritec) in the shape of a neck collar. When tracking with passive reflective markers, the tracking cameras were equipped with a custom illumination ring of 20 LEDs with wavelength of $850 \mathrm{~nm}$ (TSHG6400, Vishay Semiconductors).

\section{Close-up imaging system}

The close-up imaging system consisted of a near-infrared (NIR) enhanced CMOS Camera (MQ013RG-E2, XIMEA), mounted on the center of a custom designed two-axis gimbal, actuated by two high-power digital servo motors (MX-64, Dynamixel). The camera was equipped with a high resolution lens (Xenon, 2.0/50 mm, Schneider or a Rodagon, $4.0 / 80 \mathrm{~mm}$, Rodenstock) and an electrically tunable lens (EL-16-40-TC, Optotune). The central host machine was communicating in real time with the servos via a serial port protocol (USB2Dynamixel). The focus of the electrically tunable lens was simultaneously controlled via Ethernet protocol and a dedicated controller (TR-CL180, GIGE Vision). For recordings with mouse lemurs, the close-up camera system was protected with a transparent plexiglass half-dome (40 $\mathrm{cm}$, Lacrylic shop). 
Target illumination system

To illuminate animals for the close-up view, a high-power infrared LED (H2W5, $850 \mathrm{~nm}$ ) and reflector (10158, Roithner) or a narrow beam LED (ELJ-650-637, Roithner) was mounted either in parallel with the close-up imaging system, or separately on a second two-axis gimbal powered by two digital servo motors (MX-64, Dynamixel) and were controlled via a serial port (USB2, Dynamixel).

Remote controlled stimulus and reward boxes (RECO-box)

The RECO-box is based on a low-power WiFi-enabled microprocessor (MKR-1000, Arduino) powered by a lithium ion battery (3.7 V, 7.8Ah, Pi-shop) and interacted via a wireless UDP protocol with the host

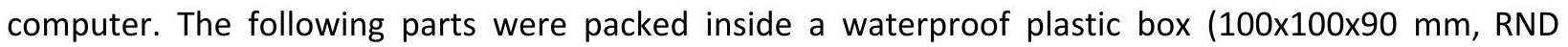
455-00123, RND Component) and held in place by a custom 3D printed insert. An optical lick-port with a single lickspout was mounted on the top cover of the plastic box. Auditory stimuli ( $9 \mathrm{KHz}$ tone) were generated by a piezo speaker (KPEG-126, Kingstate), and loud clicks by a solenoid valve (ZHO-0420L/S, Adafruit) tapping against the side wall. A green LED $(565 \mathrm{~nm})$ was activated as a visual cue, whereas an infrared LED $(750 \mathrm{~nm})$ was used to localize the position of the RECO-box by the tracking system. The liquid reward was stored in a $20 \mathrm{ml}$ glass bottle and delivered to the lick port by a peristaltic pump (OINA). The stepper motor (QSH-2818, 3.7 V, Trinamic) of the pump was controlled via a low voltage stepper driver (DRV8833, Adafruit).

\section{Software}

EthoLoop software was written in C++ using OpenCV and CUDA libraries. Real time 3D visualization of data points was done by gnuplot (www.gnuplot.info). Dynamixel SDK libraries for $\mathrm{C}++$ was used to control the servos of the close-up system(www.github.com/ROBOTIS-GIT). RECO-box were programmed with Arduino software (IDE).

\section{Animals}

\section{Mouse Lemurs}

Four adult grey mouse lemurs (Microcebus Murinus) were used for this study (Supplementary Table T1). All mouse lemurs were born and raised in the "Mouse Lemur Platform" (authorization number E-91-114-1) of the Museum of Natural History in Brunoy, France, under the supervision of the staff of the UMR CNRS-MNHN 7179-MECADEV. The procedures are in accordance with European animal welfare regulations and were reviewed by the local ethics committee ("Comité d'éthique en expérimentation animale No. 68") in Brunoy, France, by the ethics committee of the University of Geneva, Switzerland, and authorized by the French "Ministère de l'éducation nationale de l'enseignement supérieur et de la recherche". All experiments involving mouse lemurs were carried out in Brunoy. The behavioral experiments were restricted to the awake period of their circadian cycle (dark). Animals were housed socially ( 2 or 3 per cage) in cages $(50 \times 60 \times 70 \mathrm{~cm}$ ) with wooden branches, leaves and wooden nest boxes. Temperature was kept between 24 and $26{ }^{\circ} \mathrm{C}$, relative humidity was approximately $55 \%$ and artificial light cycling on 14 hours ON per day (winter period, ${ }^{55}$ ). Animals had free access to water and food consisting of pieces of fresh apples and bananas, insect larvae and a liquid food mix (condensed milk, egg yolk, cottage cheese, spice bread, baby milk powder in water and homogenized in a kitchen mixer). 


\section{Mouse lemur experimental room}

The experimental room for the mouse lemurs $(4 \mathrm{~m} \times 2 \mathrm{~m} \times 2 \mathrm{~m})$ was covered with matt black paint to avoid reflections of the infrared light sources. The mouse lemurs were allowed to freely roam in the room. The lighting condition of the room was controlled in respect to animals' day-night cycle and the temperature was maintained at $25{ }^{\circ} \mathrm{C}$. For initial tracking sessions and social interactions, the experimental room was equipped with tree branches, food platforms and wooden nest boxes (one per animal) (Fig. 1). For closed-loop experiments the room was equipped with a custom made 3D lattice maze inspired by ${ }^{37}$. The wooden sticks were $500 \mathrm{~mm}$ long and ranged in diameter from 4-24 mm.

During the days of the behavioral experiments, food availability was limited to the behavioral sessions to increase motivation. The weight and overall behavior of the animals was monitored twice a day. The animals were habituated to the RECO-boxes in their home cages, where liquid food mix (described above) was dispensed (lick triggered) after a randomly timed auditory cue ( $9 \mathrm{kHz}$ tone approximately every minute). The conditioning experiments were usually stopped after the animals performed 120 trials (reward size: $\sim 0.02 \mathrm{ml}$ of liquid food $\mathrm{mix}$ ) or when they showed a drop in motivation (ignoring the task for $>5 \mathrm{~min}$ ). Additional food was supplemented after the experiment to keep the weight stable.

\section{Mice}

Six laboratory mice (C57BI/6J, Jackson Laboratory) were used for the behavioral conditioning experiments. All experiments with mice were carried out in Geneva, Switzerland, and reviewed by the local ethics committee and authorities of the Geneva canton. During behavioral experiments, access to water was restricted to the behavioral session and limited to $1 \mathrm{ml} /$ day ${ }^{56}$. Mice were monitored daily and additional water was provided if necessary. All the experiments were carried out in the dark period of their circadian cycle. The conditioning experiments were stopped after the animals performed 100 trials (reward size: $\sim 0.01 \mathrm{ml}$ of water) or when they showed a drop in motivation (ignoring the task). Additional water was supplemented after the experiment to keep the weight stable.

\section{Surgery for optogenetic stimulation}

For optogenetic experiments using DAT-iresCre mice $\left(\mathrm{Slc}_{\mathrm{c}} \mathrm{3}^{\mathrm{tm1} 1.1(\mathrm{cre}) \mathrm{Bkmn}} / \mathrm{J}\right.$, called DAT-Cre in the manuscript), the animals were injected with rAAV5-Ef1a-DIO-hChR2(H134R)-eYFP (Addgene) in the VTA. Mice were anesthetized with a mixture of oxygen $(1 \mathrm{~L} / \mathrm{min})$ and isoflurane $3 \%$ (Baxter AG, Vienna, Austria) and placed in a stereotactic frame (Angle One; Leica, Germany). The skin was shaved, locally anesthetized with $40-50 \mu \mathrm{L}$ lidocaine $0.5 \%$ and disinfected. The animals were placed in a stereotactic frame and bilateral craniotomy was made over the VTA at the following stereotactic coordinates: lateral $\pm 0.5 \mathrm{~mm}$, posterior $-3.2 \mathrm{~mm}$, depth $-4.20 \pm 0.05 \mathrm{~mm}$ from Bregma, for a total volume of $500 \mathrm{~nL}$. The virus was expressed for 3-4 weeks and subsequently mice were implanted with optic fibers (200 $\mu \mathrm{m}$; ThorLabs) above VTA with a $10^{\circ}$ angle at the following coordinates: lateral $\pm 0.9 \mathrm{~mm}$, posterior $-3.2 \mathrm{~mm}$, depth $-3.95 \pm 0.05 \mathrm{~mm}$ from Bregma, then fixed on the skull with dental acrylic. The placement of the optical fiber and the range of virus infection were confirmed post-hoc immunostaining against Tyrosine Hydroxylase (TH) enzyme (Supplementary Fig. 10). 


\section{Experimental Procedures}

\section{Optical tracking and 3D reconstruction}

The local processing of the images of the tracking cameras on the GPUs consisted of several steps carried out in multiple parallel threads:

a) Custom color demosaicing: To spectrally separate the hues of different IR LEDs, we developed a custom demosaicing process using OpenCV and CUDA libraries. The ratio of the different color sensitivity curves (specified by the camera chip, OnSemi PYTHON 1300) were used as a lookup table to produce the false colors (Supplementary Fig. 2). This process resulted in a mapping of $660 \mathrm{~nm}$ to visible red, $750 \mathrm{~nm}$ to orange and $810 \mathrm{~nm}$ to blue.

b) 2D target position estimation: The different colors were thresholded by prespecified values for hue, saturation, and value (HSV) and the centroid of the target image blob was estimated by a projection barycenter algorithm ${ }^{23}$.

c) Communication with the central host computer: The 2D position of the centroids determined by each tracking camera/GPU was sent to the central host computer via the network using the UDP protocol.

The triangulation of simultaneously received $2 \mathrm{D}$ target information was performed in the central host computer by using a projective-invariant method, whereby the 3D target position was chosen to minimize the quadratic distance between its computed 2D projections and the measured 2D targets

$23,57,58$. Triangulation of a 3D target is possible when seen by at least two cameras, and the robustness to noise and occlusions increases with the number of cameras.

\section{Calibration of the 3D tracking system}

An initial geometric calibration phase of the system was performed by manually moving a single LED target over the entire measurement space. The recorded 2D projections were first used to estimate the 3D position of the first two cameras using the five-point algorithm ${ }^{59}$ and RANSAC estimation methods implemented in OpenCV. The 3D target position was then reconstructed using the above-mentioned triangulation algorithm. The position of each additional camera was then estimated with OpenCV's implementation of the perspective-n-point algorithm ${ }^{60}$ using the 3D triangulated points. The resulting estimated position of all cameras was then refined, using a bundle adjustment method based on a sparse Levenberg-Marquardt optimization procedure, as implemented in the SSBA software library ${ }^{61}$. As usual for 3D reconstruction algorithms, a final calibration was done to align and scale the resulting data to real-world coordinates, using a reference box object of known dimensions placed in the measurement space.

To illustrate the ability of the EthoLoop system to track fast moving objects in large scale environments $\left(>100 \mathrm{~m}^{3}\right.$ ), a commercial drone (Tello by DJl, Ryze Tech) was used (Supplementary Fig. 1, Supplementary Movie 4). The drone was steered around the plants in the room under manual control. 


\section{Calibration of the close-up system}

The liquid lens autofocus of the close-up system was calibrated manually by placing an object at $>4$ locations in front of the camera while adjusting the necessary current to achieve perfect focus. These settings were used to fit a current/distance curve the tunable lens.

The spatial parameters of the close-up system were calibrated using an semi-automated procedure. An infrared LED was placed at a random location and the close-up system started to scan the environment by spinning over the axes of both servos while capturing RAW images and thresholding in a specific range of the infrared LED (60 fps at full resolution of $1024 \times 1280$ ). This scanning stopped as soon as the LED was detected by the camera. At this point the close-up system started to move in steps of 0.087 degrees (in both axes of servos) every $500 \mathrm{~ms}$ to locate the LED at the center of the frame. The exact 3D position of the LED calculated by the host machine $(x, y, z)$, and the final angle of both servos (azimuth $\alpha$ , elevation $\beta$ ) were saved. The procedure was repeated several times, and the location $\left(x_{c}, y_{c}, z_{c}\right)$ of the close-up camera was computed by solving the system of equations:

$$
\begin{gathered}
\tan (\beta)=\frac{x-x_{c s}}{y-y_{c s}} \\
\tan (\alpha)=\frac{z_{c s}}{\sqrt{\left(x-x_{c s}\right)^{2}+\left(y-y_{c s}\right)^{2}}}
\end{gathered}
$$

Finally, the angles of the servo motors were trigonometrically calculated by the host machine based on the spatial position of the target and respective location of the close-up camera and communicated via the serial port. In parallel, the distance from the target to the close-up camera was calculated and sent via Ethernet to the GIGE Vision controller to focus the tunable lens on the object using the above-mentioned calibration curve.

\section{Evaluation of the EthoLoop system performance}

\section{D tracking and real-time behavior analysis latency}

The latency for 3D tracking was defined by the time between the appearance of a target and the 3D reconstruction. A microcontroller (Arduino Mega) with an infrared LED (750 nm) was connected to the host PC and placed in the tracking area. The host program was modified to command the microcontroller to illuminate the LED at repeated intervals, and to measure the time elapsed until reception of the corresponding 3D position measurement of the LED. This procedure was repeated 1000 times at random intervals. The average value gives a high estimate of the actual latency of the system, considering the added latency of commanding the microcontroller to turn on the infrared LED (Fig. 1f).

The latency of the real-time behavioral analysis was defined by the time between the appearance of an LED in front of the closeup system (detected by the real-time DeepLabCut as an LED with pre trained network) and the receiving of coordinate and likelihood by the host machine (Fig. 1f). The procedure to turn on the LED and calculate the time difference was identical to the one described above (tracking latency). 


\section{RECO box communication latency}

The latency to communicate with a RECO-box was defined as the time between a command was sent from the host machine and the reception of that command by the RECO-box. For this purpose 1000 commands at random intervals were sent to a RECO-box to turn on the infrared LED. Once the LED was turned on, the cameras send the 2D coordinate to the host machine for 3D reconstruction. The time difference between sending of the command and the reconstruction of LED's 3D position was calculated by the host machine. These measurements were then subtracted from the mean value of 3D reconstruction latency (single target, Fig. 1f, light-red histogram) to obtain communication latency with the RECO-box (Fig. 1f, blue histogram).

\section{Rate of 3D tracking and real-time behavior analysis}

The interval for 3D tracking was defined as the time between two subsequent 3D reconstructions. We recorded the time between reception of subsequent 3D data measurements, repeated over a 60s tracking session with one, two and three targets. (Fig. 1g). Real-time behavior analysis interval was defined as the time between subsequent detection and labeling of body parts. For this purpose, a moving doll was placed in front of the close-up camera. The body parts were labeled with the real-time DeepLabCut (using the pre-trained network) and the time between subsequent labeled frames were recorded over 60 seconds.

\section{Positional accuracy}

An infrared LED was placed on the edge of a circular turntable (Thorens, TD160) spinning at 33 and 45 rounds per minute (rpm). The difference between the actual radius and the radius of the circle of the trajectory tracked by the EthoLoop system was reported as the positional error estimated for the tracking system (Supplementary Fig. 1d).

\section{Real-time detection of behaviors}

To detect specific behaviors (rearing, Fig. 4) in the stream of close-up images the following steps were carried out:

a) Real-time labeling of body parts. The close-up images were labeled using a customly programmed real-time version of DeepLabCut software ${ }^{43}$. Close-up images were initially streamed at full resolution $(1280 \times 1024)$ to a computer (DELL, Optiplex 990-Intel Core i7, equipped with a GeForce GTX 1080 Ti graphics card). These images were resized to $640 \times 512$ and saved using OpenCV functions. To reduce latency and increase speed, the images were further resized to $320 \times 256$ and labeled by the DeepLabCut software. The body parts included: eyes, nose, forelimbs, hindlimbs, and the base of the tail. The deep neural network was trained with hand annotated images ( $<400$ frames) from previous recordings under similar lighting conditions.

b) Once an image was labeled, the frame number, the coordinates of the body parts and their likelihood were saved in a single string. This string was transmitted in real-time as a UDP packet to the main host machine. A special thread in a multithreaded tracking program was assigned to receive and parse the string. 
c) Real-time detection of posture: The spatial relationship of individual real-time labeled body parts were used to define a given posture (Fig. 4a). Specifically, rearing was detected if one of the following conditions were met:

i) Difference between the nose and hind limbs on the y axis was more than 200 pixels and both lines connecting the nose to the hind limbs make an angle of less than 20 degrees with the $y$ axis.

ii) Difference between one of the eyes and hind limbs in y axis was more than 200 pixels and both lines connecting one eye to the hind limbs make an angle of less than 20 degrees with the y axis.

d) Spatial constraints: In the experiments where the posture had to be carried out at a specific location (Fig. 4b-g), a predefined virtual sphere $(200 \mathrm{~mm})$ placed at a chosen location. The reward was only triggered when the posture was detected within the sphere.

\section{Real-time control of RECO-box}

The RECO-box was controlled via a wifi-Arduino. After establishing the connection to the central host computer via the wireless network, the Arduino was waiting for incoming UDP packets. Each received packet was decoded and the corresponding function (reward, noise, etc.) was activated. The delay of this process was around $\sim 2.5 \mathrm{~ms}$ (Fig. 1f, blue histogram). The RECO-boxes were attached to different branches of the lattice maze, and their position was determined by the tracking system using a built-in infrared LED (750 nm). RECO-boxes indicated the availability of a reward as a 1 second $9 \mathrm{KHz}$ sound repeated at $0.5 \mathrm{~Hz}$. The actual reward was delivered, upon arrival of the animal within $10 \mathrm{~cm}$ of the RECO-box.

\section{Close-loop behavioral experiments}

Mouse lemur foraging

Up to 4 to 5 RECO-box were distributed at different locations inside the 3D lattice maze (Fig. 2, Supplementary Fig. 4). Their activation was set in either pre-defined or random sequences. In experiments with defined sequences, a subsequent RECO-box was activated when the animal reached a distance of $20 \mathrm{~cm}$ from the previous one. In experiments with random activation, a different RECO-box was chosen at random to be activated after a variable delay ( 0 to $60 \mathrm{~s}$ ) as soon as the animal reached a distance of $1 \mathrm{~m}$ to the previous activated box. In both cases the animal was given $40 \mathrm{~s}$ to collect the reward from the active feeder.

\section{Mouse lemur 3D place conditioning}

Upon the entrance of the animal to a $15 \mathrm{~cm}$ radius sphere surrounding the selected location ("place") a RECO-box was activated. The animal was given 20 seconds to collect the reward from the active RECO-box.

\section{Mouse lemur behavioral conditioning (Rearing)}

Upon executing the defined behaviour (rearing) at a specified location a RECO-box was activated. The animal was given 30 seconds to collect the reward from the active RECO-box.

\section{Mouse place conditioning}

A RECO-box was placed on a wooden platform $(110 \times 60 \mathrm{~cm})$, and the feeder was localized by the tracking system with an infrared $(750 \mathrm{~nm})$ LED. The activation of a feeder was signalled by a click sound, 
and liquid reward $(0.01 \mathrm{ml}$ of $0.1 \%$ sucrose in water) was provided upon arrival near the feeder. Initially the mice were habituated to the feeder, by providing drops of rewards at random intervals.

\section{Mouse behavioral conditioning (Rearing)}

In order to detect rearing on hind limbs, a threshold on the $z$ axis was defined. Once rearing was detected, a feeder was activated and the mouse had 10 seconds to collect the reward. On day 1 of the experiment, the mouse had to perform rearing anywhere on the platform within $20 \mathrm{~cm}$ of the feeder. On day 2, a three-level stage was placed on the wooden platform and the mouse had to perform rearing on top of the stage. To initially encourage rearing, a small bell was hung by a string from the ceiling, requiring the mouse to rear to explore the object. The bell and string were removed after the 10th trial.

\section{Mouse optogenetic place and behavioural conditioning}

For the optogenetic conditioning experiments the mice were connected to a flexible optical fiber (FC/PC $200 \mu \mathrm{m}$ fiber, $2 \mathrm{~m}$, ThorLabs) hanging from the ceiling. The light source was a fiber-pig-tailed laser of 473 $\mathrm{nm}$ (50 mW, OBIS, Coherent) controlled via a WiFi-enabled microprocessor (MKR-1000, Arduino) circuit. The maximal power of the fiber output was $20 \mathrm{~mW}$ (measured in continuous mode using a power meter (Field Mate, Coherent)). Stimulation consisted of 8 pulses of 4 ms duration, repeated at 30 $\mathrm{Hz}$ (output ranging from $8-12 \mathrm{~mW}$ ). During the place conditioning experiment, mice were optogenetically stimulated upon entrance to the defined location $(20 \mathrm{~cm}-25 \mathrm{~cm}$ radius). The optogenetic stimulation continued for a maximum of 8 consecutive bursts if the mouse remained inside the target area. The place conditioning experiments were stopped after the animals received $\sim 900$ burst stimulations. During the behavioural conditioning experiments, mice were optogenetically stimulated upon executing the defined behaviour (rearing) detected with the real-time DeepLabCut software. The experiments were stopped after the animals received $\sim 450$ burst stimulations.

\section{Mouse lemur electrophysiological recordings}

\section{Electrodes and drive}

Custom-made tetrode wire bundles were produced by twisting and heating four individual $12.7 \mu \mathrm{m}$ Tetrode Nickel-Chrome Wires covered with Easy Bond XTC (Sandvik). Tetrodes were inserted and glued into polyamide tubing $(0.14 \mathrm{~mm}$ diameter) inside the moving part of a custom designed 3D-printed microdrive. The electrode tips were cut and gold-plated with Sifco 5355 (mixed 1:1 with PEG solution and carbon nanotubes, Neuralynx) to lower the impedance. The free end of the tetrode was connected to a miniature connector (Omnetics) via a custom designed interface board. The tetrode array was lowered by turning the M1 screw of he microdrive.

\section{Electrophysiology setup}

Extracellular recordings were performed using custom wireless data acquisition logger hardware (Spike Gadgets) together with Trodes software (SpikeGadgets). Acquired data were sampled at $20 \mathrm{kHz}$ and broad-band filtered between $0.1 \mathrm{~Hz}$ and $10 \mathrm{kHz}$. Data were further filtered post hoc between $600 \mathrm{~Hz}$ and $6 \mathrm{kHz}$ for spike extraction. Spikesorting was carried out with semi-automated SpikingCircus and Kilosort algorithms. 


\section{Surgery}

One day before the surgery the animal was administered antibiotics (Ceftriaxon, $50 \mu \mathrm{g} / \mathrm{g}$, intramuscular) and housed individually without food. Anaesthesia was induced in a small plexiglass box with a continuous flow of a mixture of oxygen with $5 \%$ Isoflurane (Vetflurane, Virbac). After induction the animal was removed from the box and placed in a custom designed stereotactic frame. Eyes were protected with Lacryvisc cream and whiskers and tongue were covered with Vaseline. Isoflurane was decreased (2.5-1\%) while breathing rate and toe pinch reflexes were continuously monitored. The following drugs were administered: Bupremorphine $(3 \mu \mathrm{g} / \mathrm{g}$, subcutaneous $)$, Dexamethasone $(0.8 \mu \mathrm{g} / \mathrm{g}$ intramuscular), Ceftriaxon (50 $\mathrm{\mu g} / \mathrm{g}$, intramuscular), Carprofen $(22 \mu \mathrm{g} / \mathrm{g}$, intramuscular), Rapidocaine (100-150 $\mu \mathrm{l}$, locally injected). The area of the surgical procedure was disinfected sequentially with $70 \%$ Ethanol, Betadine and Chlorhexidine 1\%. The cranial bones were exposed after skin incision. The periosteum was gently removed with cotton swabs and the skull surface was dried and roughened to improve glue bonding. The area for the craniotomy was located at $0.5 \mathrm{~mm}$ anterior and $3 \mathrm{~mm}$ lateral from the interaural midpoint and was chosen based on coordinates from atlases of the mouse lemur brain ${ }^{62-64}$. A thin layer of cyanoacrylate (5011, ERGO) was applied on the skull and holes for ground wires and craniotomy for electrodes were made using a dental drill. The microdrive was placed on the skull and fixed with dental acrylic (Lang Dental). The skin was closed with stitches and the animal was placed on a heating blanket for recovery. The animal was administered analgesic Bupremorphine ( 3 $\mu \mathrm{g} / \mathrm{g}$, subcutaneous) for the following week and antibiotics (Ceftriaxon (50 $\mu \mathrm{g} / \mathrm{g}$, intramuscular) for a total of 9 days.

\section{Synchronization of electrophysiology and tracking}

To synchronize the 3D position from tracking system and the electrophysiology recording, a signal was sent from the 3D tracking program to a Wifi Arduino (MKR-1000) via the UDP protocol. The timing of this signal was recorded in the tracking software. Upon arrival of the UDP packet at the Wifi Arduino a digital pulse was sent to the Main Control Unit of the electrophysiology acquisition system (Spike gadgets) that synchronizes the recordings in the wireless head-stage via radio frequency pulses. The timing of these pulses were extracted offline and aligned with the 3D tracking.

\section{Post hoc data analysis}

All the position data, timing and signals (stimulations and rewards) were stored in a text file. All post data analysis was done in R using OpenGL libraries (www.r-project.org).

\section{Position data filtering}

Image artefacts produced for instance by reflections of bright and metal surfaces can cause false detections in the 3D tracking system. This false detections appears as sudden jumps between the actual location of the LED and the reflecting surface. To correct for these artifacts:

- Points that appeared outside the tracking boundary were excluded.

- Sudden jumps (speed $>5 \mathrm{~m} / \mathrm{s}$ ) inside the tracking space were excluded and the trajectories interpolated.

- Finally, the tracking data was smoothed over all 3 axes using locally weighted regression (LOWESS function in R). 
From the filtered position data, velocity and acceleration on each axis at each time point was computed using a moving average over a 60 ms sliding window.

\section{Movement categorization (ethograms)}

Five behavioral types were automatically extracted from the animal's movements using the following mutually exclusive rules:

- 'Short Quiet': velocity was below $20 \mathrm{~cm} / \mathrm{s}$ for less than 2 seconds.

- 'Long Quiet': velocity was below $20 \mathrm{~cm} / \mathrm{s}$ for more than 2 seconds.

- 'Running': velocity was above $20 \mathrm{~cm} / \mathrm{s}$, and cumulative displacement was over $10 \mathrm{~cm}$ otherwise categorize into above 'Quiet' states depending on duration.

- 'Jumping': vertical acceleration equal to Earth's gravity $\pm 3 \frac{\mathrm{m}}{\mathrm{s}^{2}}$

- 'Falling': vertical acceleration equal to Earth's gravity $\pm 3 \frac{\mathrm{m}}{\mathrm{s}^{2}}$, vertical displacement above $20 \mathrm{~cm}$ and the average horizontal velocity below $50 \mathrm{~cm} / \mathrm{s}$.

\section{Distance between animals during quiet states}

In tracking sessions with two animals, when both animals were at long quiet state, we computed the 3D distance between them and the amount of time they were at this distance. A histogram of distances binned at $20 \mathrm{~cm}$ intervals was plotted where the y axis represents the percent of time spent at each interval.

\section{State transitions}

State transitions were defined by the event when a lemur changed from one state to another state. The distances between two mouse lemurs were calculated when one of them (Lemur A) transitions from 'long quiet' to 'running' state $\left(S T_{Q \rightarrow R}\right)$ while the other animal was in 'running' state (Lemur B). These distances were all binned in $200 \mathrm{~mm}$ intervals and the number of $S T_{Q \rightarrow R}$ at each interval was calculated. At each distance interval, the probability of $S T_{Q \rightarrow R}$ of Lemur A upon approaching Lemur B was estimated as the number of $S T_{Q \rightarrow R}$ divided by the total duration where distance between two animals was within the interval. In order to detect whether Lemur B was approaching Lemur A or moving away, the distance between them was also calculated 2 seconds prior to $S T_{Q \rightarrow R}$ of Lemur A.

\section{Analysis of closed-loop experiment with RECO-Box}

To assess learning in the closed-loop experiments (place and behavior conditioning in mice and mouse lemurs) we analyzed the first 50 activation of the RECO-Box and this data was divided into three equal time bins of termed "beginning", "middle" and "end".

\section{Analysis the operant conditioning by optogenetic stimulation}

The place conditioning sessions were analyzed up to the moment when the mouse had spent a total of 350 s in the conditioned location (with the exception of mouse M3, conditioned place 1, where the experiment was terminated at a total $188 \mathrm{~s}$ in the conditioned location). The maps in Fig. $\mathbf{5}$ and Supplementary Fig. 8. represent the probability density of the location of the mouse during the last third of conditioning session.

In the behavioral conditioning session the time until the first 200 rearings divided into three equal time bins termed "beginning", "middle" and "end". 


\section{$3 D$ reconstruction of lattice maze}

All 60 cubes connecting different branches of the lattice maze were numbered. Each branch in the lattice maze was defined by its thickness $(4 \mathrm{~mm}, 6 \mathrm{~mm}, 12 \mathrm{~mm}$ and $24 \mathrm{~mm})$ and the cube numbers connecting them to each other. The 3D coordinate of the first cube was manually measured in the real-world coordinates and set as the base for reconstructing the lattice maze in 3D. The 3D position of all cubes in the lattice maze was reconstructed relative to the base cube (considering the constant 3D distance between neighboring cubes). Finally the whole lattice maze was reconstructed by adding the branches while knowing their connecting cubes.

\section{Analysis of Electrophysiology}

Spike rates for the 3D color coded trajectories (Fig. $6 a$ \& $6 c$ \& $6 e$ ) are calculated using a sliding window $(500 \mathrm{~ms})$ in which we calculated the average number of spikes.

The trajectory of the mouse lemurs was divided into bins of $13 \mathrm{~cm}$ displacement length. Spike rates are assigned to each bin by counting the number of spikes divided by the time taken to complete the displacements. The floor (Fig. 7b) was defined as the first $30 \mathrm{~cm}$ above ground.

For cells with spatially restricted firing at a specific branch, a virtual $10 \mathrm{~cm}$ sphere was initially placed at the center of the branch. All passages through the sphere with their respective firing rate were aligned based on their minimum distance to the center of the sphere (time zero is when the minimum distance happens for each passage, Fig. 6d). Iteratively, the sphere was moved along the branch and the firing rate of all trajectories were calculated and aligned. The sphere movement was stopped once the average firing rate of all trajectories reached its maximum value when passing the center of the sphere. This center of the sphere at this location was considered as the "center".

For a more detailed analysis (Fig. 6e-j), the direction of displacement was also added as a factor, creating directional vectors. The vectors were color coded based on the spike rate. To quantify the movement along branches, the area around the connecting cubes (radius of $5 \mathrm{~cm}$ ) were excluded from this analysis. The nomenclature of the directions (north, south, east, west, up, down) were defined with regards to the $\mathrm{X}, \mathrm{Y}$ and $\mathrm{Z}$ axis of the lattice maze and independent of the real World coordinates.

In vivo estimation of tetrode position in the mouse lemur brain

The position of the tetrode bundles was determined by the 3D reconstruction of in vivo computer tomographic (CT) scans acquired for the anesthetized animals (isoflurane anesthesia as detailed above; scan time $=3 \mathrm{~min}$.) at the MicroCT platform of the University Paris Descartes. The position of the brain areas with respect to the skull and the tetrodes (reconstructed by the CT data) were estimated based on the manual alignment with existing data from high resolution MRI atlas ${ }^{64}$, Supplementary Fig. 10) using the AMIRA (Thermo Fisher Scientific) software.

\section{Statistical analysis}

For all two-way ANOVA tests, the Levene's test was used to test the homogeneity of variances and Shapiro-Wilk test was performed on the ANOVA residuals to test the normality assumption. For the analysis of the directional-branch related cell (Fig. 7j) we performed two independent non-parametric 
one-way ANOVA (Kruskal-Wallis test) since both assumptions of normality and homogeneity of variance were not meeting.

\section{Code availability}

All the codes for tracking (GPU units and Host machine), RECO-boxes and real-time labeling of body parts are available at www.huberlab.org/EthoLoop/software. R codes used for analysis are available upon request.

\section{Data availability}

All figure data is provided in the Supplementary Data section and the original raw data is available upon request. 


\section{References:}

1. Tinbergen, N. The Study of Instinct Clarendon Press. Oxford 195, I (1951).

2. Krakauer, J. W., Ghazanfar, A. A., Gomez-Marin, A., Maclver, M. A. \& Poeppel, D. Neuroscience Needs Behavior: Correcting a Reductionist Bias. Neuron 93, 480-490 (2017).

3. Neuroethology and Behavioral Physiology: Roots and Growing Points. (Springer, Berlin, Heidelberg, 1983).

4. Hölscher, C., Schnee, A., Dahmen, H., Setia, L. \& Mallot, H. A. Rats are able to navigate in virtual environments. J. Exp. Biol. 208, 561-569 (2005).

5. Dombeck, D. A., Harvey, C. D., Tian, L., Looger, L. L. \& Tank, D. W. Functional imaging of hippocampal place cells at cellular resolution during virtual navigation. Nat. Neurosci. 13, $1433-1440$ (2010).

6. Keller, G. B., Bonhoeffer, T. \& Hübener, M. Sensorimotor mismatch signals in primary visual cortex of the behaving mouse. Neuron 74, 809-815 (2012).

7. Harvey, C. D., Collman, F., Dombeck, D. A. \& Tank, D. W. Intracellular dynamics of hippocampal place cells during virtual navigation. Nature 461, 941-946 (2009).

8. Minderer, M., Harvey, C. D., Donato, F. \& Moser, E. I. Neuroscience: Virtual reality explored. Nature 533, 324-325 (2016).

9. Aghajan, Z. M. et al. Impaired spatial selectivity and intact phase precession in two-dimensional virtual reality. Nat. Neurosci. 18, 121-128 (2015).

10. Aronov, D. \& Tank, D. W. Engagement of neural circuits underlying 2D spatial navigation in a rodent virtual reality system. Neuron 84, 442-456 (2014).

11. Stowers, J. R. et al. Virtual reality for freely moving animals. Nat. Methods 14, 995-1002 (2017).

12. Dombeck, D. A., Khabbaz, A. N., Collman, F., Adelman, T. L. \& Tank, D. W. Imaging large-scale neural activity with cellular resolution in awake, mobile mice. Neuron 56, 43-57 (2007).

13. Sofroniew, N. J., Cohen, J. D., Lee, A. K. \& Svoboda, K. Natural whisker-guided behavior by 
head-fixed mice in tactile virtual reality. J. Neurosci. 34, 9537-9550 (2014).

14. Radvansky, B. A. \& Dombeck, D. A. An olfactory virtual reality system for mice. Nat. Commun. 9, 839 (2018).

15. Fry, S. N., Bichsel, M., Müller, P. \& Robert, D. Tracking of flying insects using pan-tilt cameras. J. Neurosci. Methods 101, 59-67 (2000).

16. Fry, S. N., Rohrseitz, N., Straw, A. D. \& Dickinson, M. H. TrackFly: virtual reality for a behavioral system analysis in free-flying fruit flies. J. Neurosci. Methods 171, 110-117 (2008).

17. Straw, A. D., Branson, K., Neumann, T. R. \& Dickinson, M. H. Multi-camera real-time three-dimensional tracking of multiple flying animals. J. R. Soc. Interface 8, 395-409 (2011).

18. Bath, D. E. et al. FlyMAD: rapid thermogenetic control of neuronal activity in freely walking Drosophila. Nat. Methods 11, 756-762 (2014).

19. Romero-Ferrero, F., Bergomi, M. G., Hinz, R. C., Heras, F. J. H. \& de Polavieja, G. G. idtracker.ai: tracking all individuals in small or large collectives of unmarked animals. Nature Methods 16, 179-182 (2019).

20. Weissbrod, A. et al. Automated long-term tracking and social behavioural phenotyping of animal colonies within a semi-natural environment. Nat. Commun. 4, 2018 (2013).

21. de Chaumont, F. et al. Computerized video analysis of social interactions in mice. Nat. Methods $\mathbf{9}$, 410-417 (2012).

22. Matsumoto, J. et al. A 3D-Video-Based Computerized Analysis of Social and Sexual Interactions in Rats. PLOS ONE 8, e78460 (2013).

23. Ballesta, S., Reymond, G., Pozzobon, M. \& Duhamel, J.-R. A real-time 3D video tracking system for monitoring primate groups. J. Neurosci. Methods 234, 147-152 (2014).

24. Khan, Z., Herman, R. A., Wallen, K. \& Balch, T. An outdoor 3-D visual tracking system for the study of spatial navigation and memory in rhesus monkeys. Behav. Res. Methods 37, 453-463 (2005).

25. Tsoar, A. et al. Large-scale navigational map in a mammal. Proc. Natl. Acad. Sci. U. S. A. 108, 
E718-24 (2011).

26. Yartsev, M. M. \& Ulanovsky, N. Representation of Three-Dimensional Space in the Hippocampus of Flying Bats. Science 340, 367-372 (2013).

27. Hong, W. et al. Automated measurement of mouse social behaviors using depth sensing, video tracking, and machine learning. Proceedings of the National Academy of Sciences 112, E5351-E5360 (2015).

28. Shemesh, Y. et al. Correction: High-order social interactions in groups of mice. Elife 3, e03602 (2014).

29. De Chaumont, F., Ey, E., Torquet, N. \& Lagache, T. Live Mouse Tracker: real-time behavioral analysis of groups of mice. BioRxiv (2018).

30. Strauss, R., Schuster, S. \& Götz, K. G. Processing of artificial visual feedback in the walking fruit fly Drosophila melanogaster. J. Exp. Biol. 200, 1281-1296 (1997).

31. Del Grosso, N. A., Graboski, J. J., Chen, W. \& Hernández, E. B. Virtual Reality system for freely-moving rodents. BioRxiv (2017).

32. Buccino, A. P. et al. Open source modules for tracking animal behavior and closed-loop stimulation based on Open Ephys and Bonsai. J. Neural Eng. 15, 055002 (2018).

33. Lim, J. \& Celikel, T. Real-time contextual feedback for close-loop control of navigation. J. Neural Eng. (2019). doi:10.1088/1741-2552/ab2ffa

34. Stephens, D. W., Brown, J. S. \& Ydenberg, R. C. Foraging: Behavior and Ecology. (University of Chicago Press, 2008).

35. Krebs, J. R. \& Davies, N. B. Behavioural Ecology: An Evolutionary Approach. (John Wiley \& Sons, 2009).

36. Silcox, M. T. \& López-Torres, S. Major Questions in the Study of Primate Origins. Annual Review of Earth and Planetary Sciences 45, 113-137 (2017).

37. Grobéty, M.-C. \& Schenk, F. Spatial learning in a three-dimensional maze. Anim. Behav. 43, 
1011-1020 (1992).

38. Jovalekic, A. et al. Horizontal biases in rats' use of three-dimensional space. Behav. Brain Res. 222, 279-288 (2011).

39. Skinner, B. F. The Behaviour of Organisms. (1938).

40. Breland, K. \& Breland, M. A field of applied animal psychology. Am. Psychol. 6, 202-204 (1951).

41. Pryor, K. Don't shoot the dog! The new art of teaching and training (revised edition). (Bantam Books, 1999).

42. Wiltschko, A. B. et al. Mapping Sub-Second Structure in Mouse Behavior. Neuron 88, 1121-1135 (2015).

43. Mathis, A. et al. DeepLabCut: markerless pose estimation of user-defined body parts with deep learning. Nat. Neurosci. 21, 1281-1289 (2018).

44. Tsai, H.-C. et al. Phasic firing in dopaminergic neurons is sufficient for behavioral conditioning. Science 324, 1080-1084 (2009).

45. O'keefe, J. \& Nadel, L. The hippocampus as a cognitive map. (Oxford: Clarendon Press, 1978).

46. Wilson, M. A. \& McNaughton, B. L. Dynamics of the hippocampal ensemble code for space. Science 261, 1055-1058 (1993).

47. Ulanovsky, N. Neuroscience: how is three-dimensional space encoded in the brain? Curr. Biol. 21, R886-8 (2011).

48. Pereira, T. D. et al. Fast animal pose estimation using deep neural networks. Nat. Methods 16, 117-125 (2019).

49. Picq, J.-L. Aging affects executive functions and memory in mouse lemur primates. Exp. Gerontol. 42, 223-232 (2007).

50. Finkelstein, A. et al. Three-dimensional head-direction coding in the bat brain. Nature 517, 159-164 (2015).

51. Kolling, N., Behrens, T. E. J., Mars, R. B. \& Rushworth, M. F. S. Neural mechanisms of foraging. 
Science 336, 95-98 (2012).

52. Szuts, T. A. et al. A wireless multi-channel neural amplifier for freely moving animals. Nat. Neurosci. 14, 263-269 (2011).

53. Gershenfeld, N., Krikorian, R. \& Cohen, D. The Internet of things. Sci. Am. 291, 76-81 (2004).

54. Perret, M., Gomez, D., Barbosa, A., Aujard, F. \& Théry, M. Increased late night response to light controls the circadian pacemaker in a nocturnal primate. J. Biol. Rhythms 25, 186-196 (2010).

55. Perret, M. Change in photoperiodic cycle affects life span in a prosimian primate (Microcebus murinus). J. Biol. Rhythms 12, 136-145 (1997).

56. Guo, Z. V. et al. Procedures for behavioral experiments in head-fixed mice. PLoS One 9, e88678 (2014).

57. Harltey, A. \& Zisserman, A. Multiple view geometry in computer vision. (2006).

58. Hartley, R. I. \& Sturm, P. Triangulation. Comput. Vis. Image Underst. 68, 146-157 (1997).

59. Nistér, D. An efficient solution to the five-point relative pose problem. IEEE Trans. Pattern Anal. Mach. Intell. 26, 756-777 (2004).

60. Nister, D. An efficient solution to the five-point relative pose problem. in 2003 IEEE Computer Society Conference on Computer Vision and Pattern Recognition, 2003. Proceedings. 2, II-195 (2003).

61. Zach, C. Robust Bundle Adjustment Revisited. in Computer Vision - ECCV 2014 772-787 (Springer, Cham, 2014).

62. Bons, N. A Stereotaxic Atlas of the Grey Lesser Mouse Lemur Brain (Microcebus Murinus). (Elsevier, 1998).

63. Dhenain, M., Ruffins, S. W. \& Jacobs, R. E. Three-dimensional digital mouse atlas using high-resolution MRI. Dev. Biol. 232, 458-470 (2001).

64. Nadkarni, N. A., Bougacha, S., Garin, C., Dhenain, M. \& Picq, J.-L. Digital templates and brain atlas dataset for the mouse lemur primate. Data Brief 21, 1178-1185 (2018). 



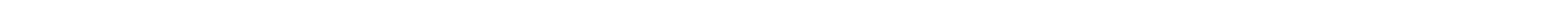


$\begin{array}{llll}\mathbf{a} & \mathbf{b} & \mathbf{c} & \mathbf{d}\end{array}$

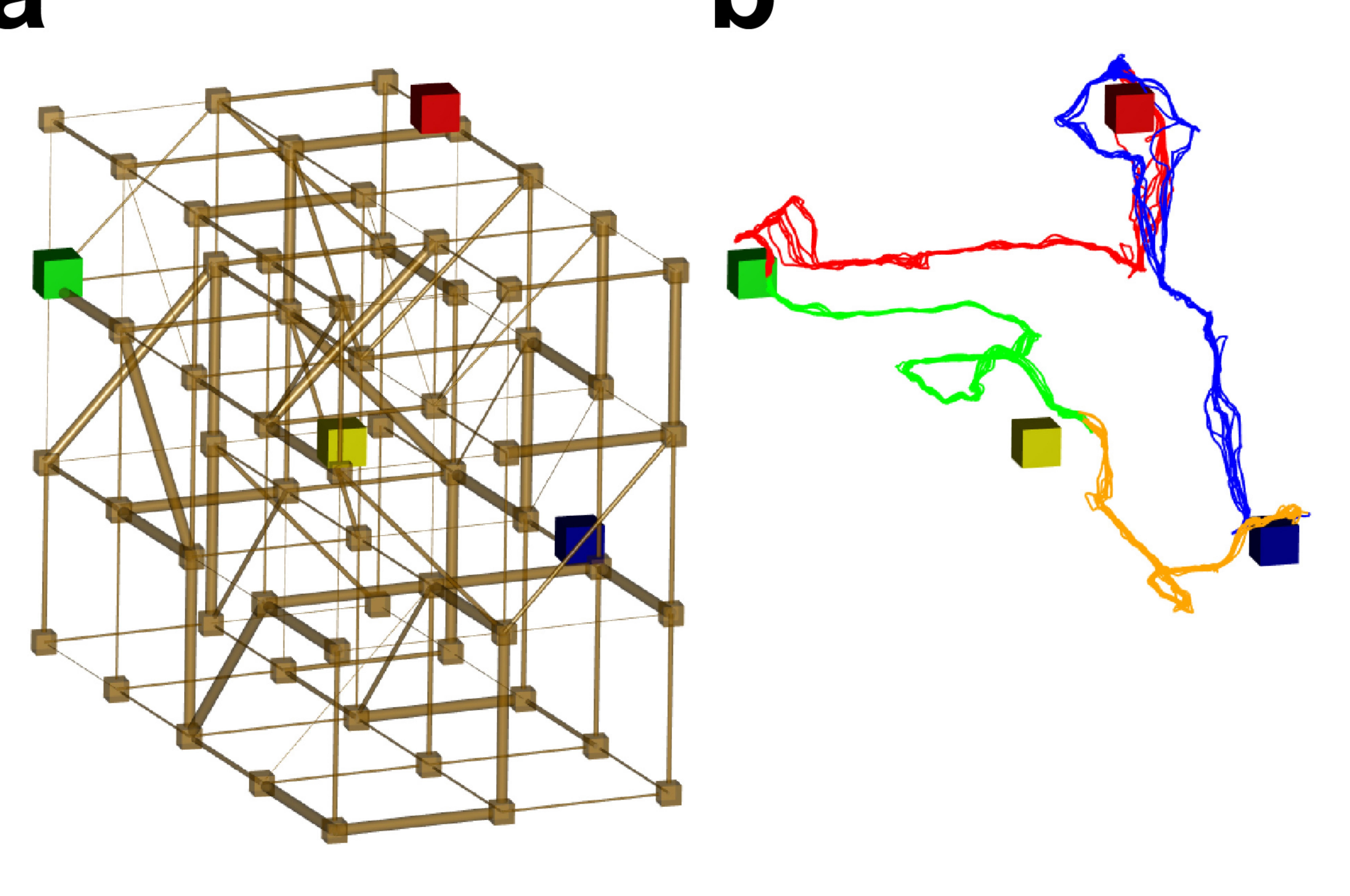

Dour RECO-boxes distributed inside the lattice maze

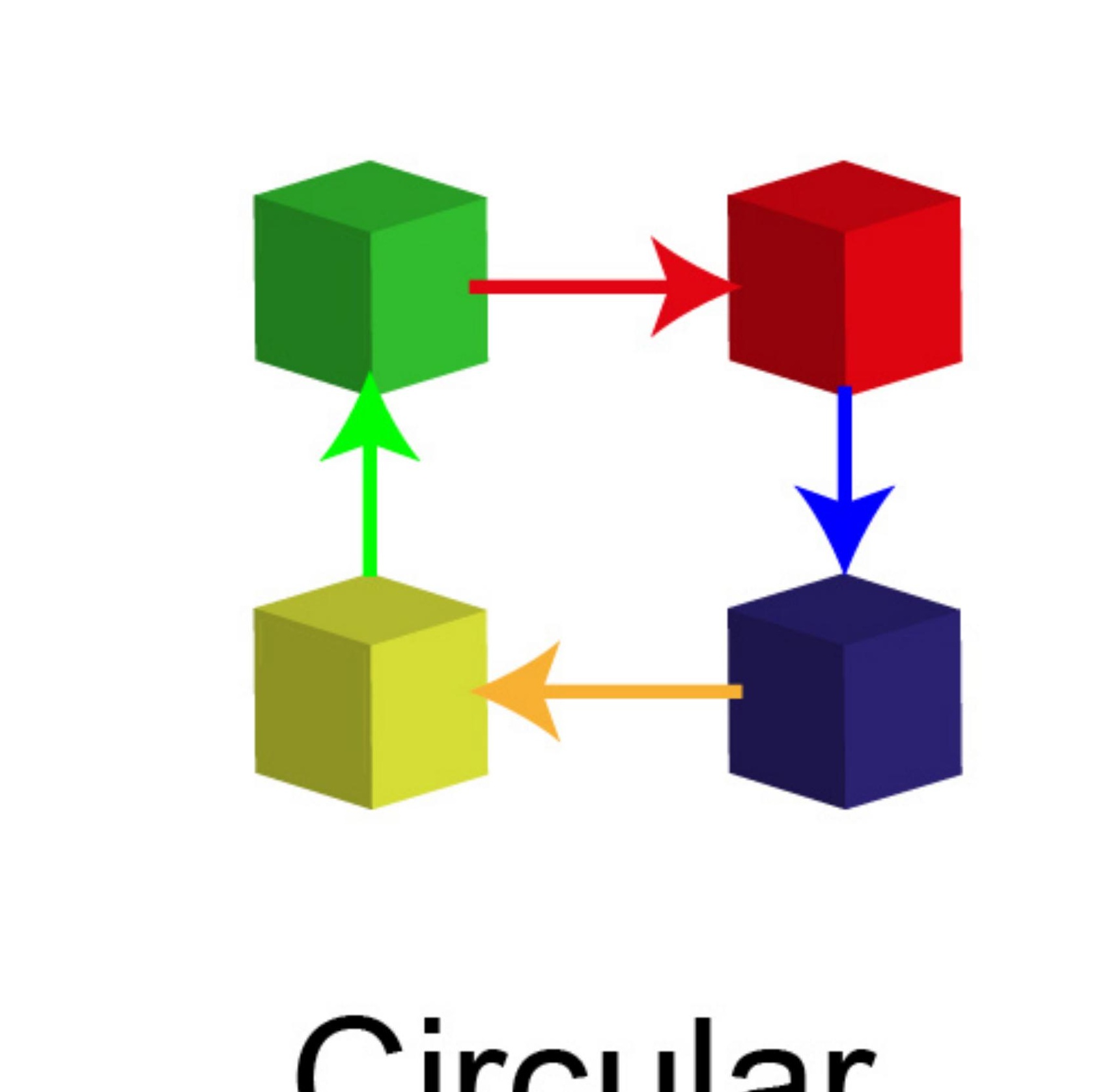

Circular activation order
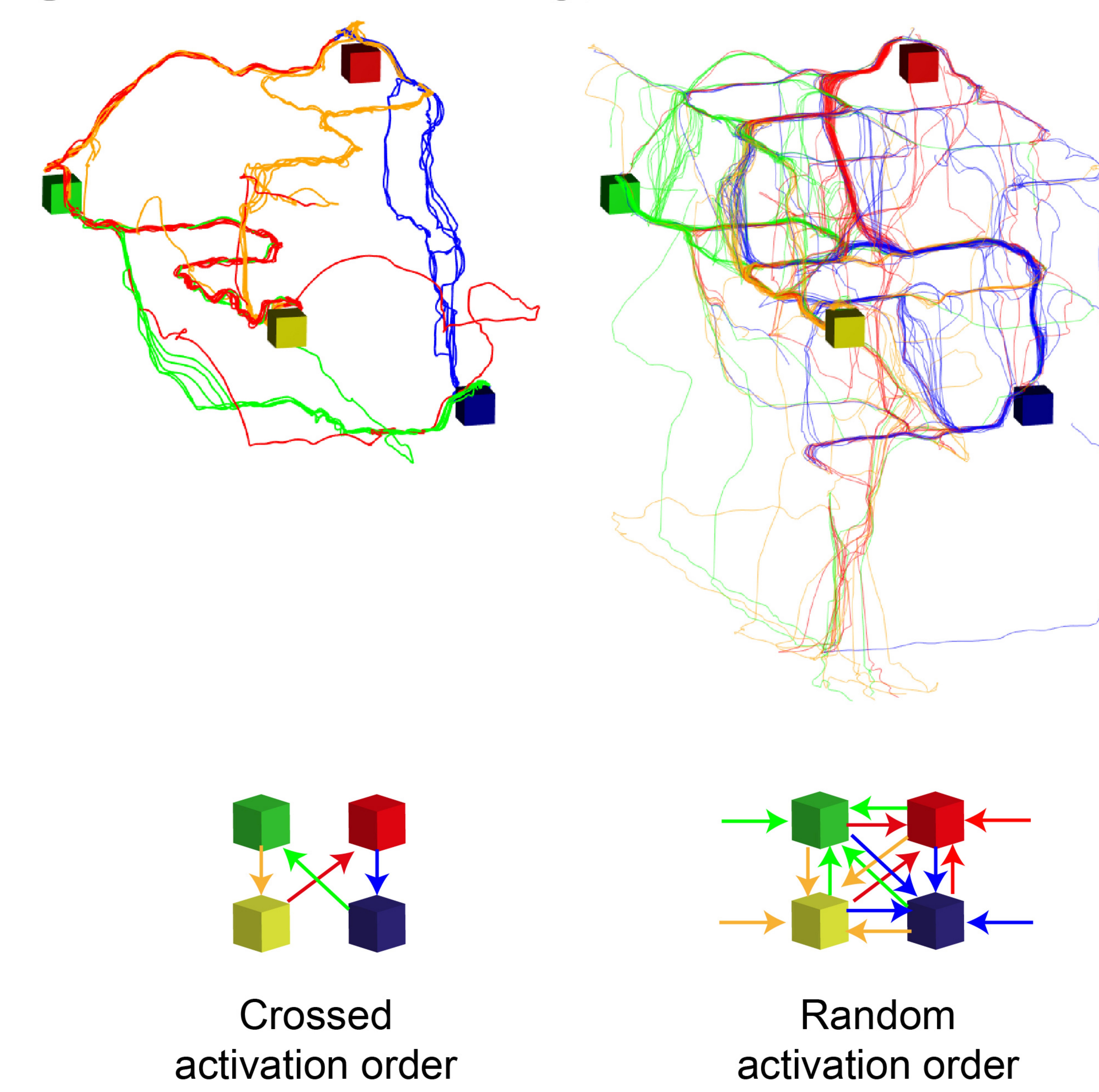

$\mathbf{e}$

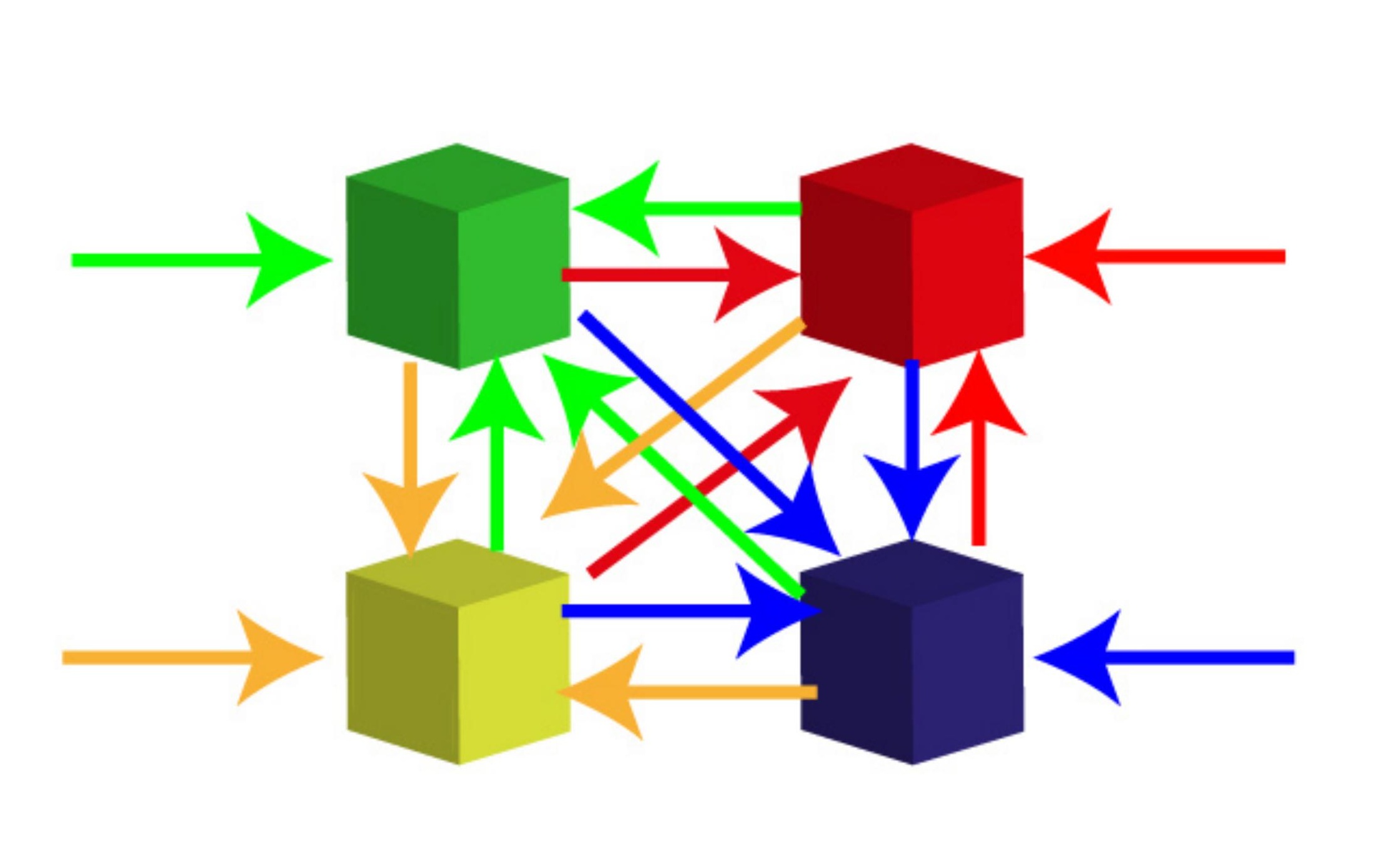

Random activation order

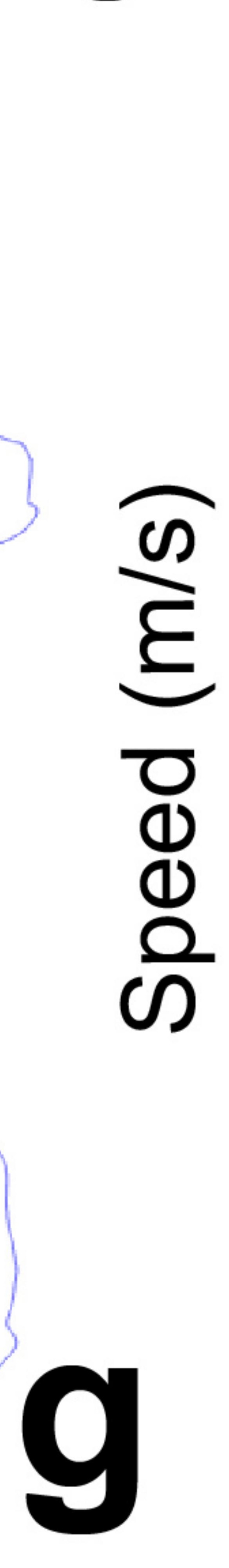

g

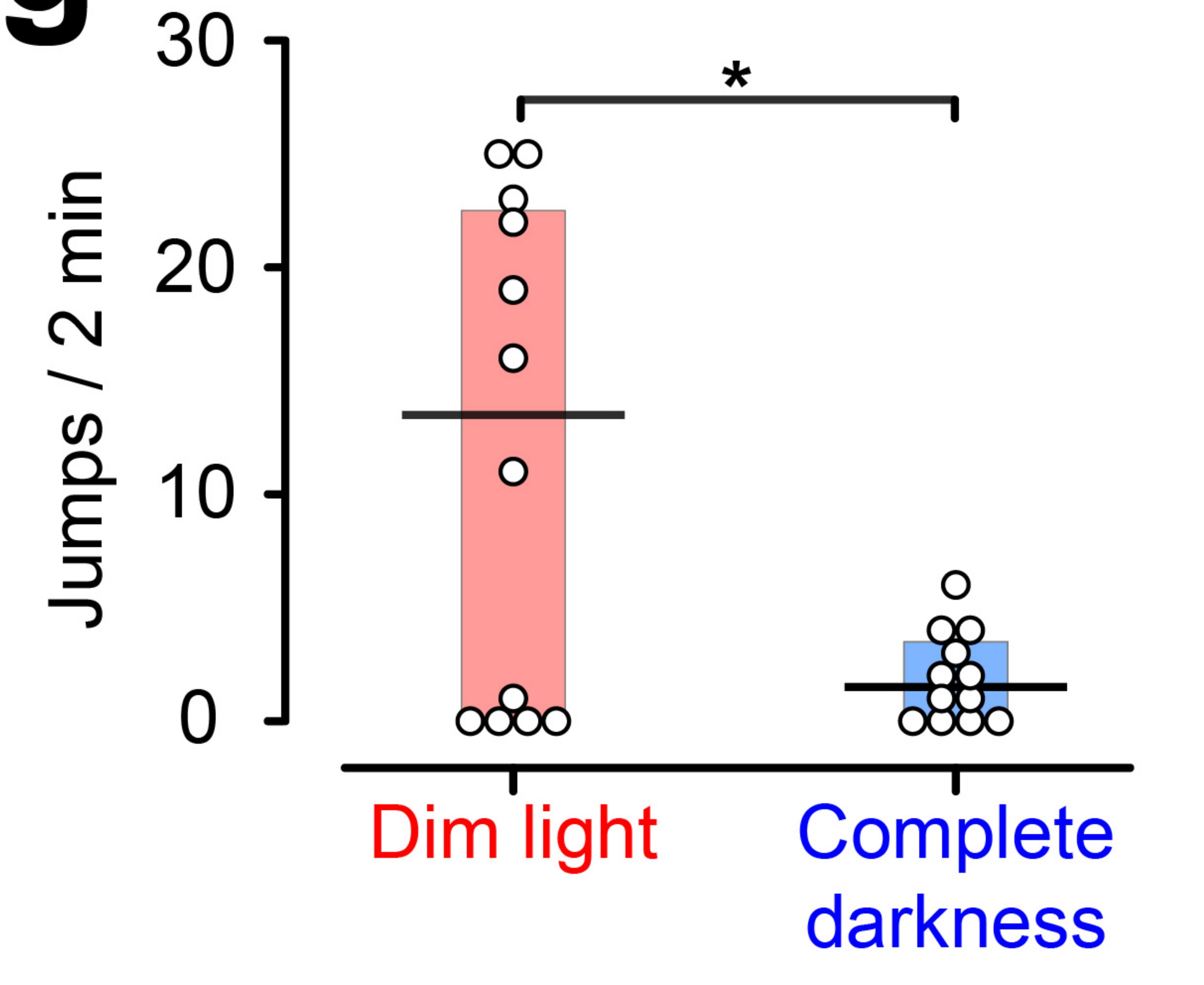

f Complete darkness

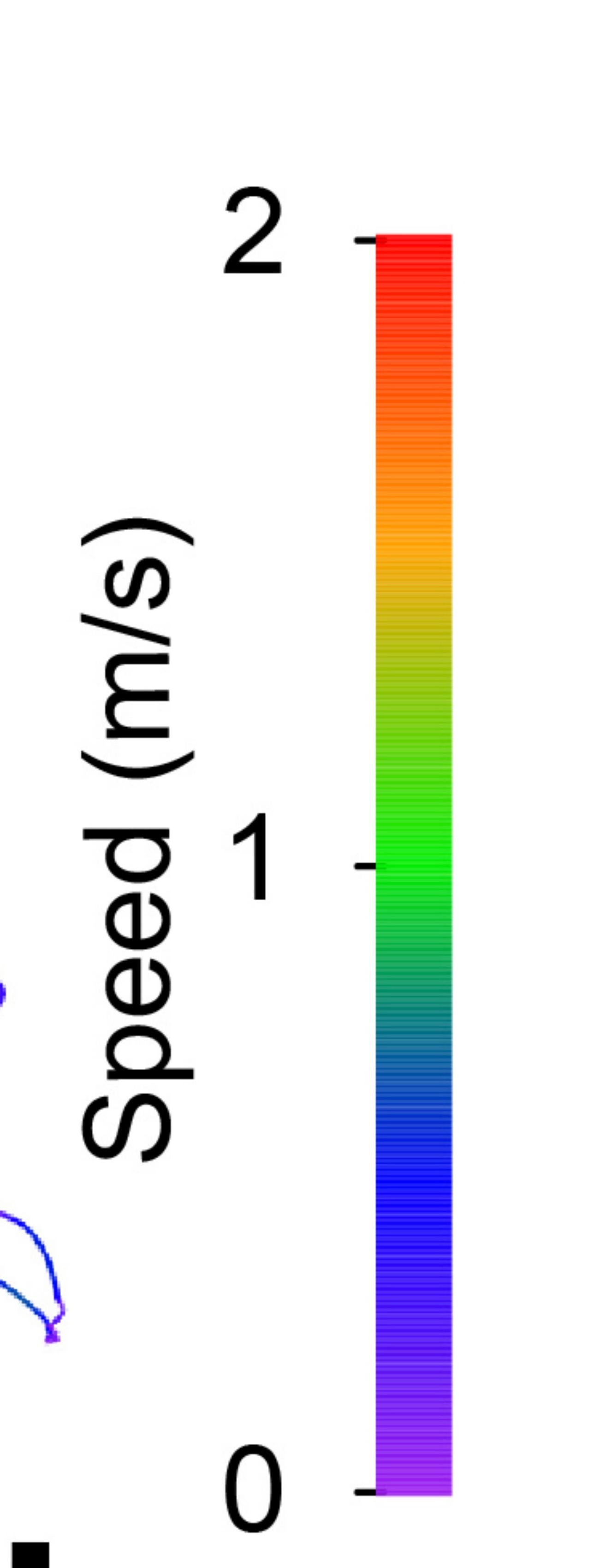

h

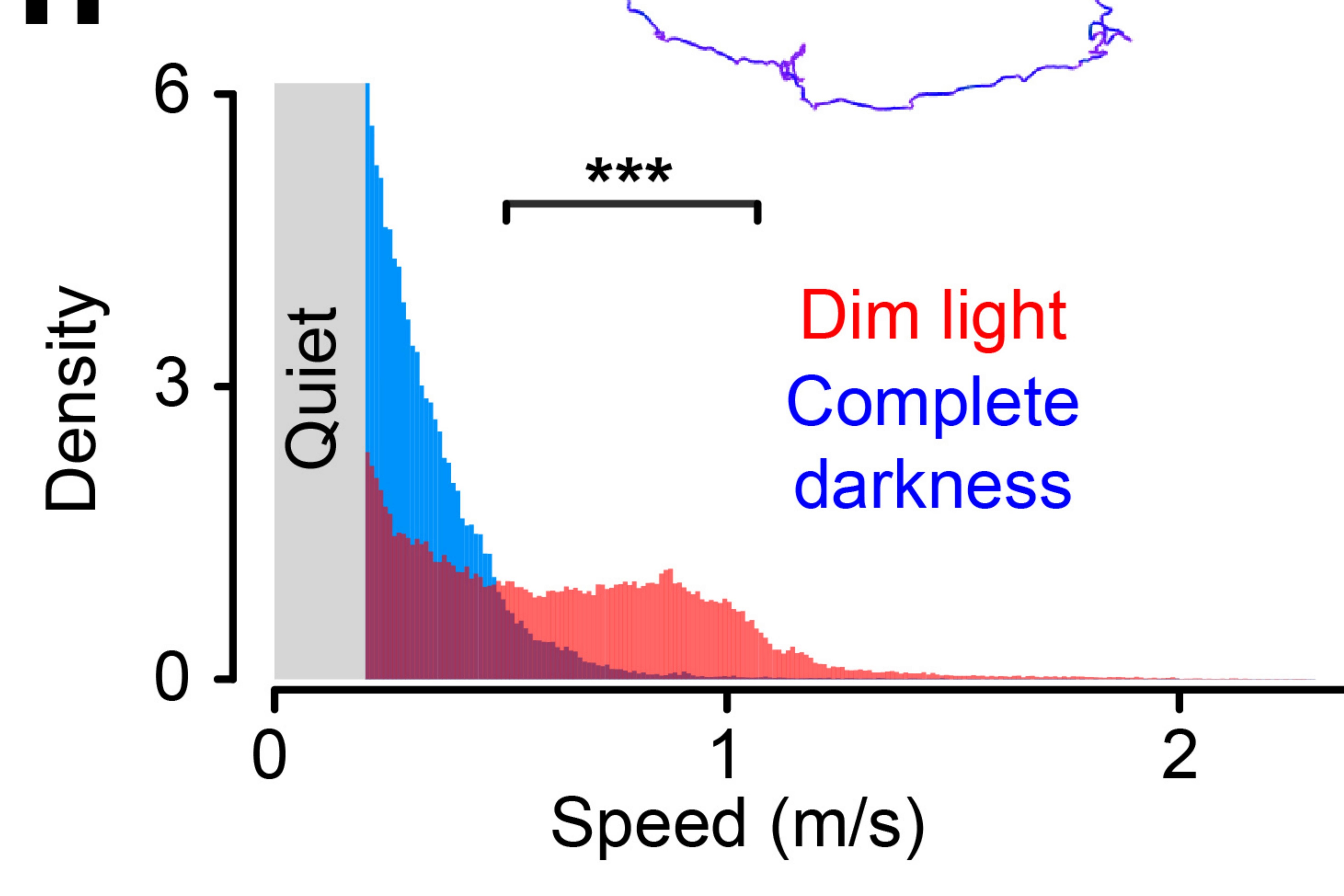


$\mathbf{a}$

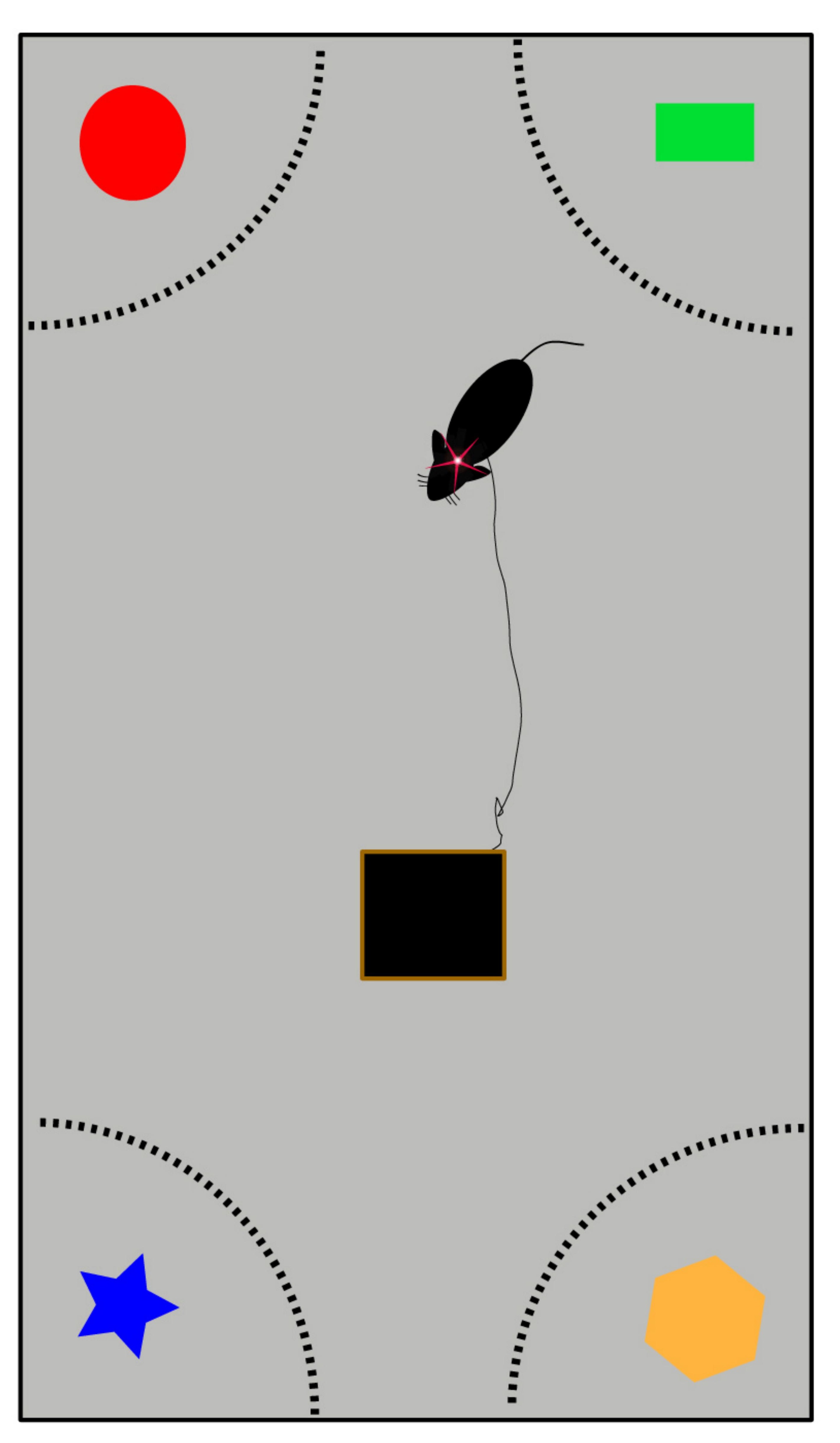

$\square$ Objects

Reward box

e

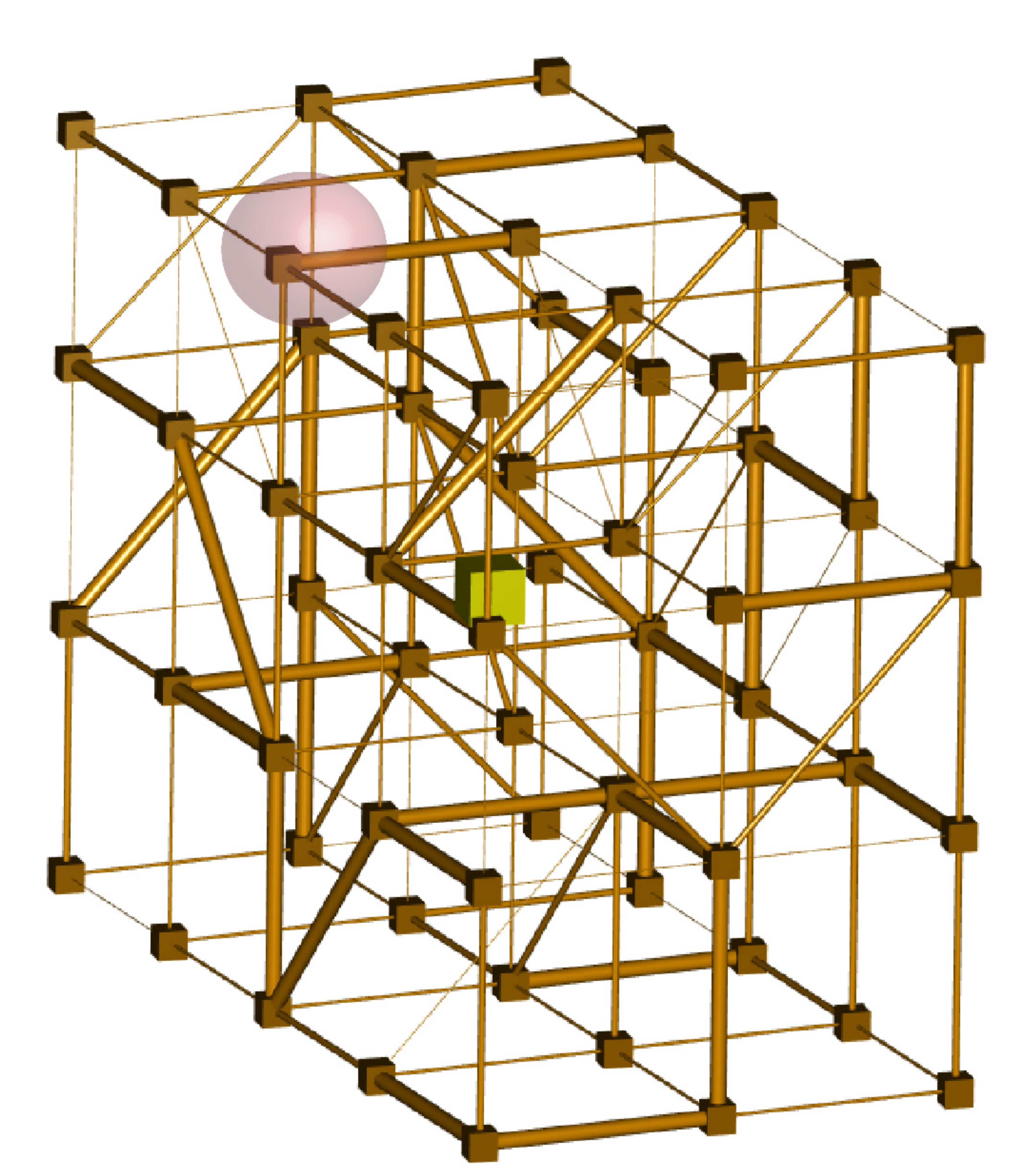

Conditioned place 1

Reward box
C

일ำ

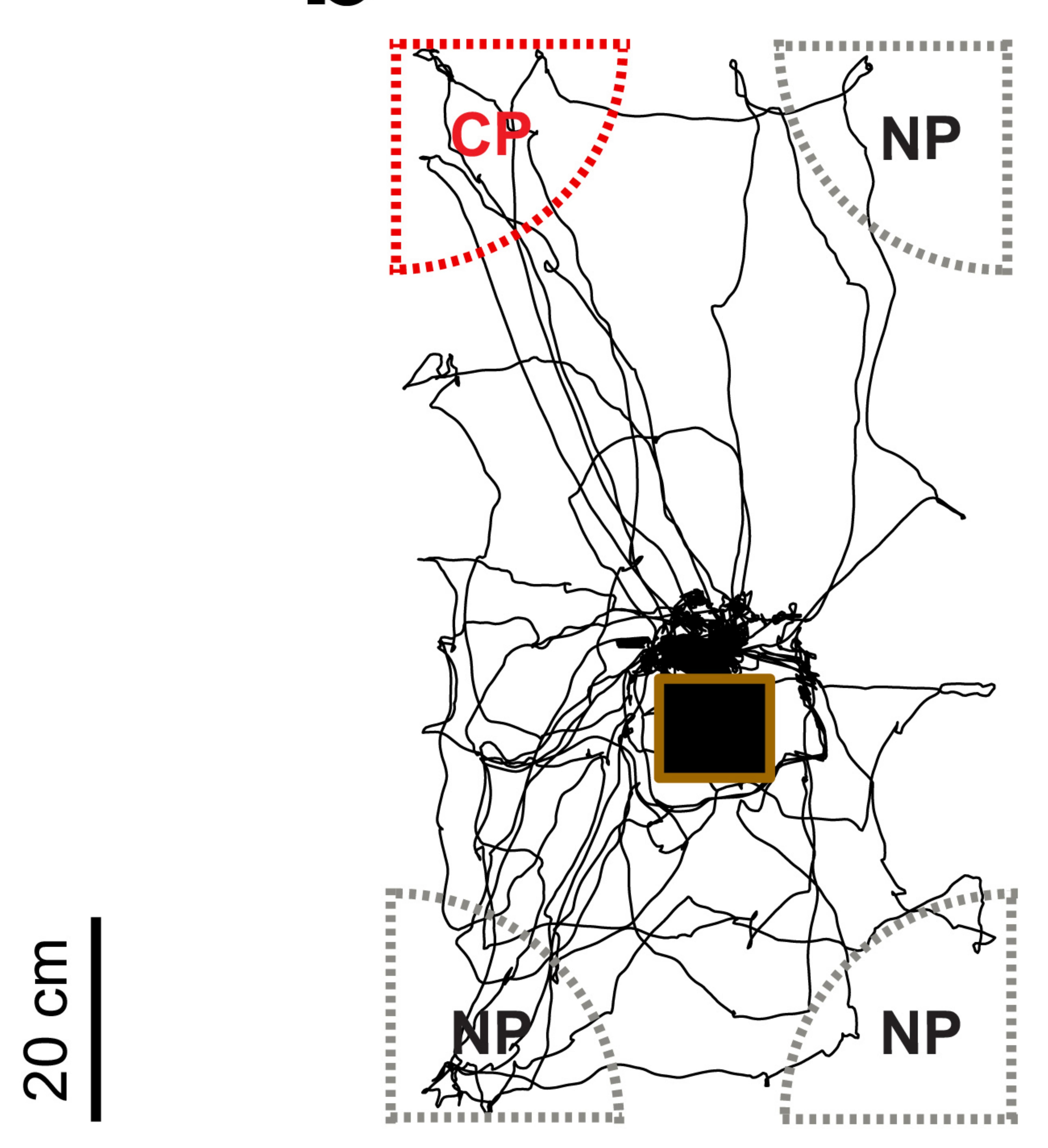

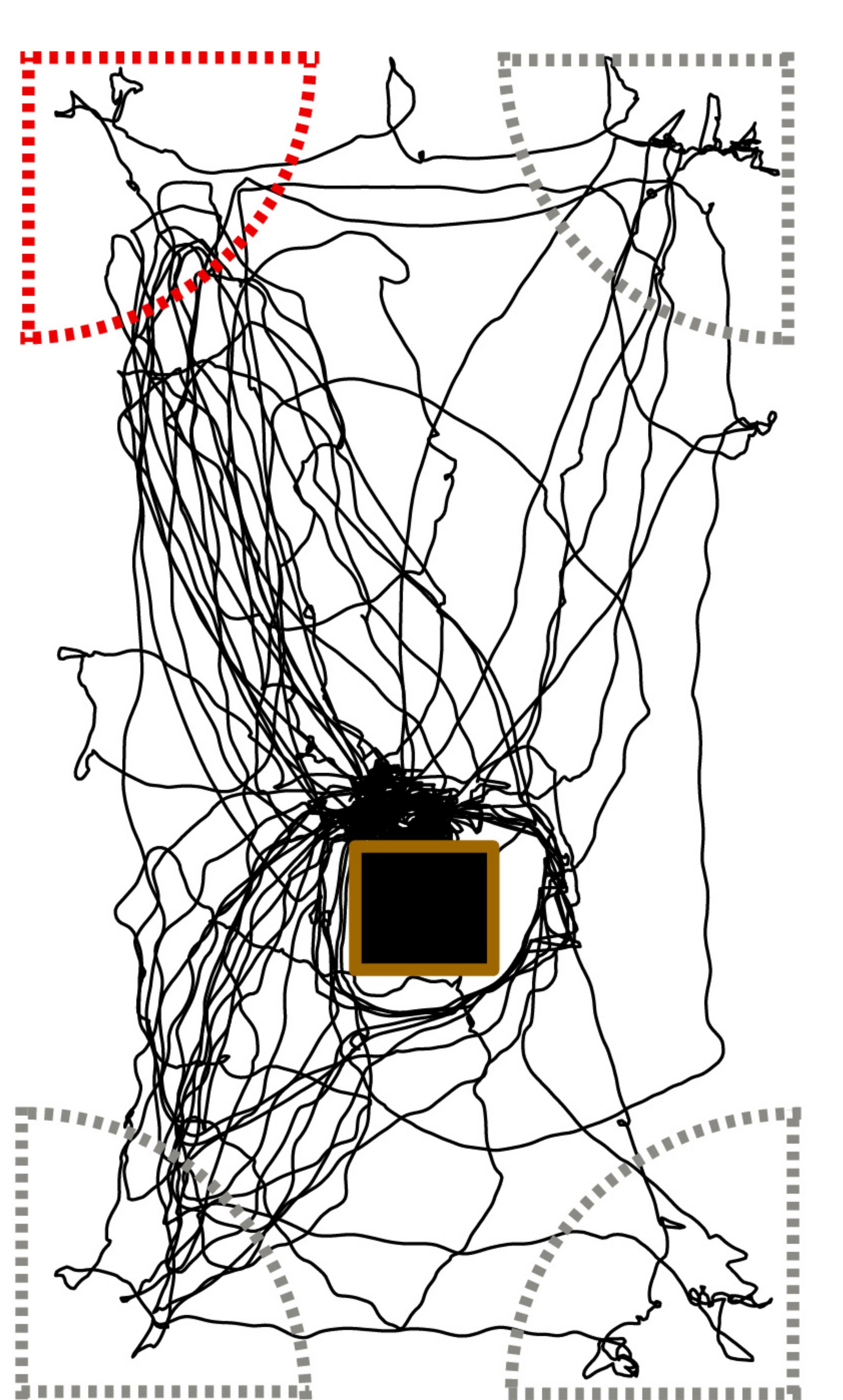

Middle

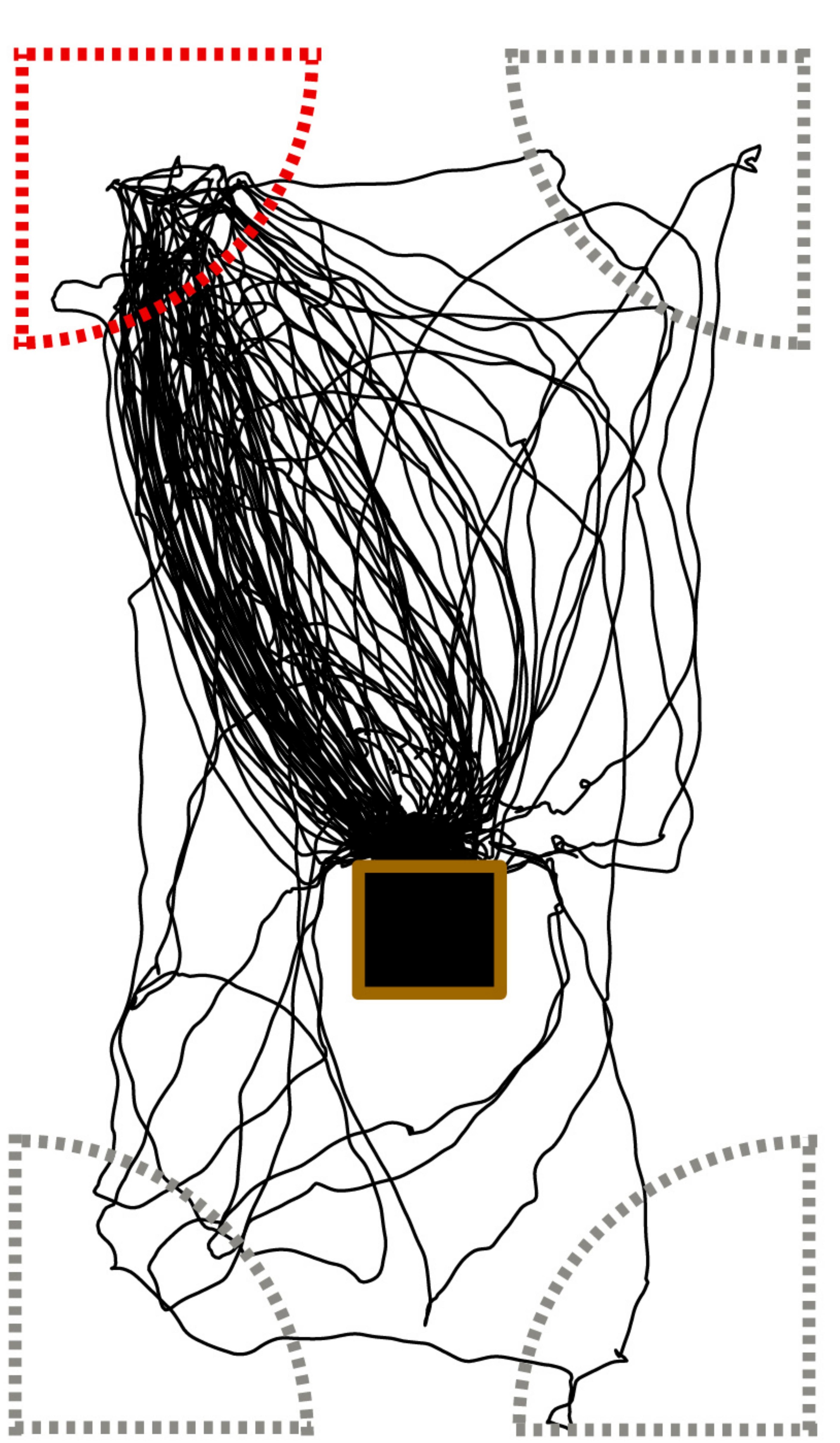

End

d

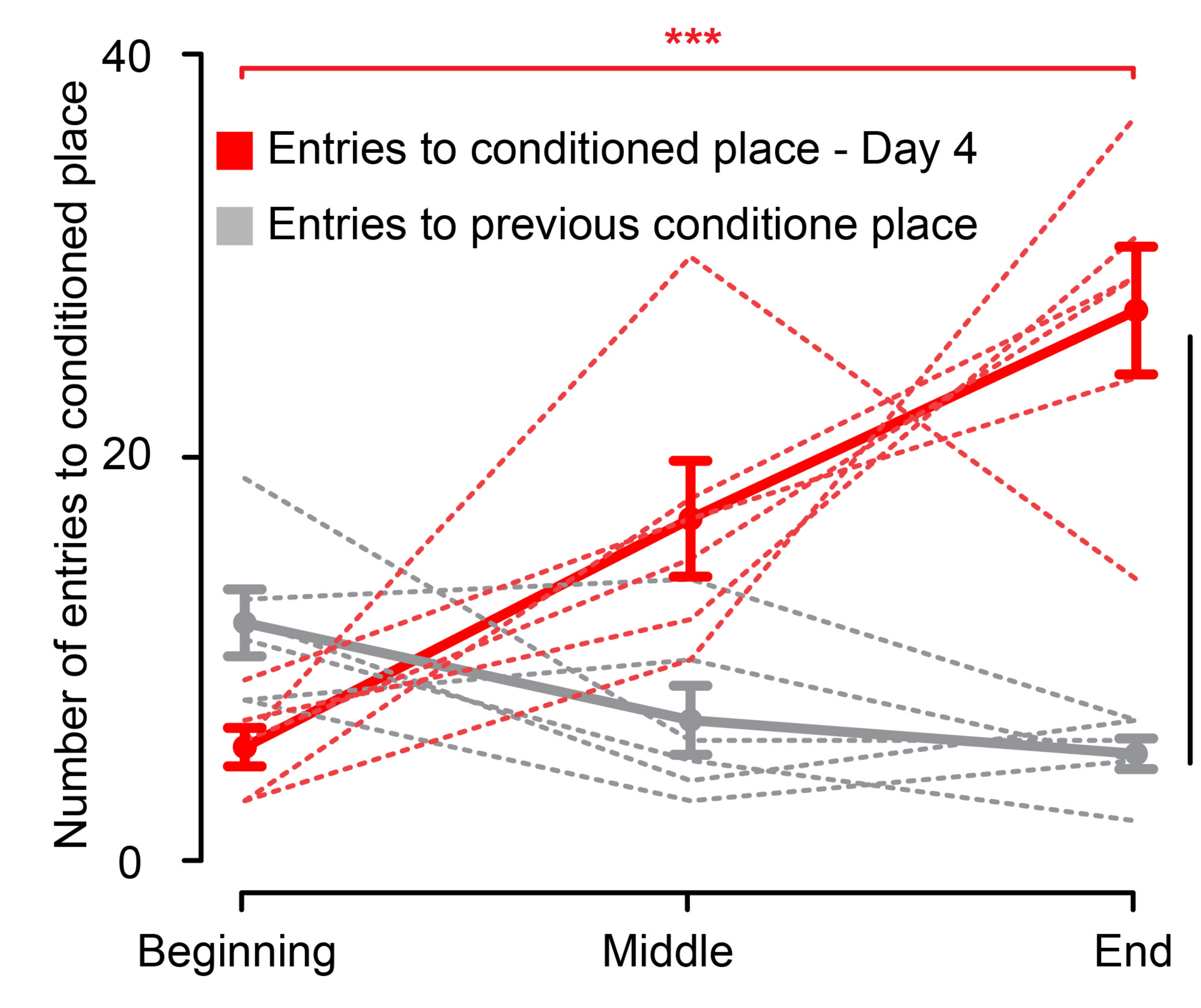

h
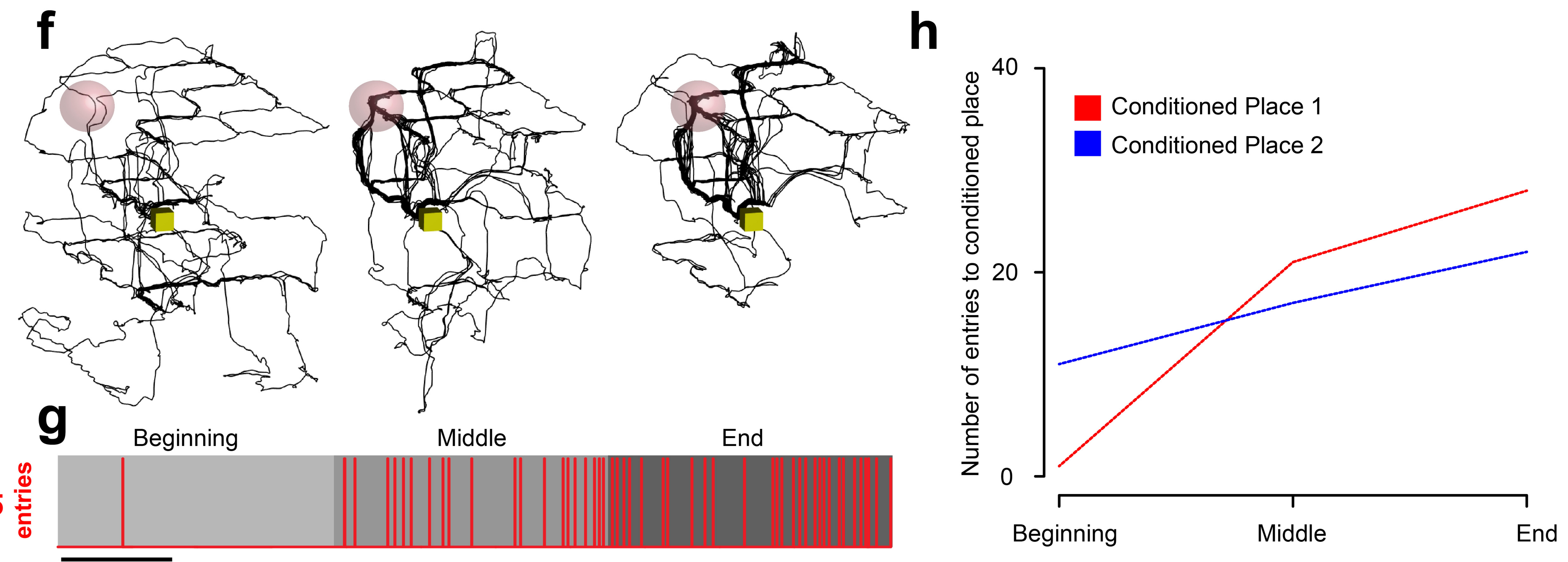
a Model of target behavior

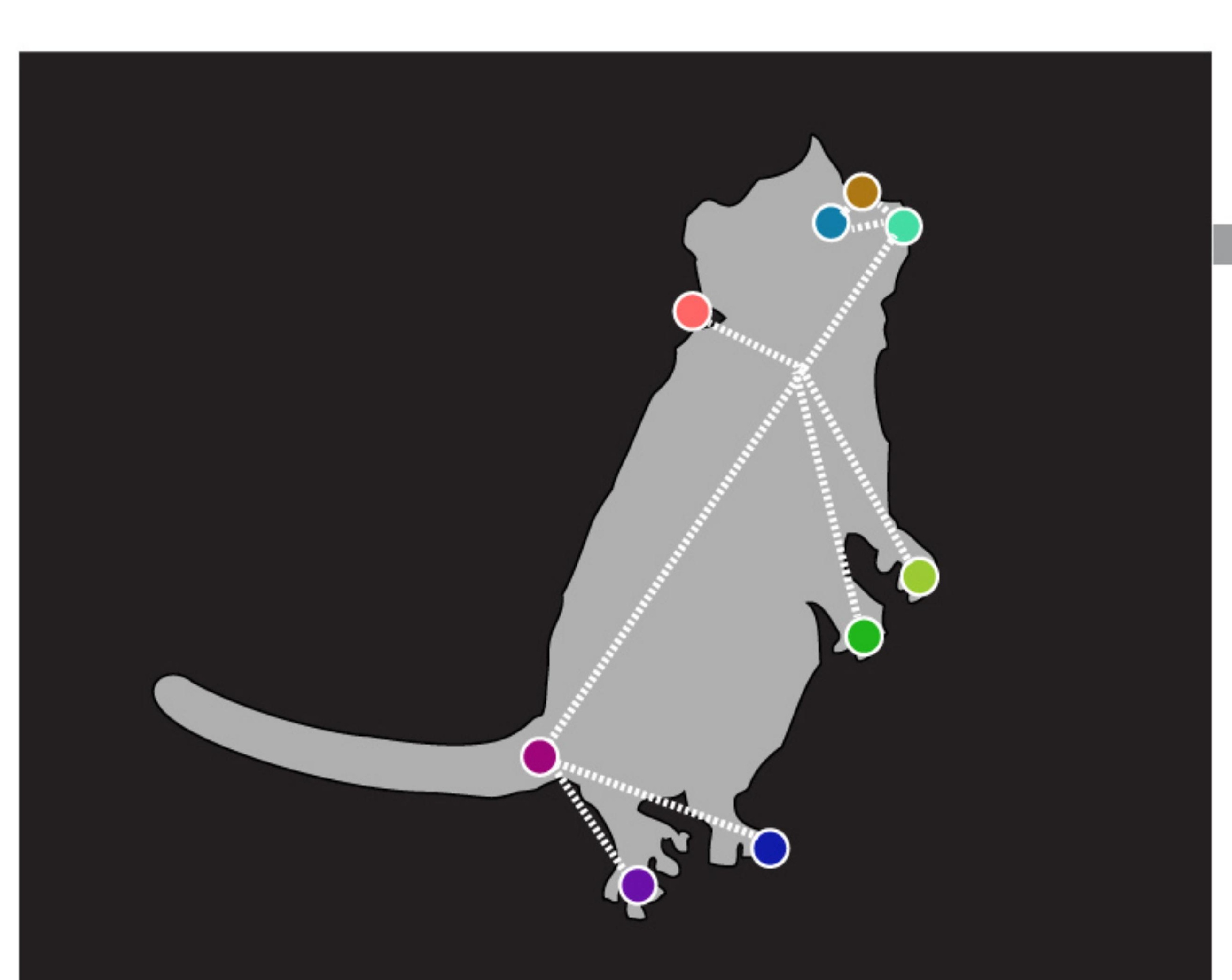

Behavioral detection

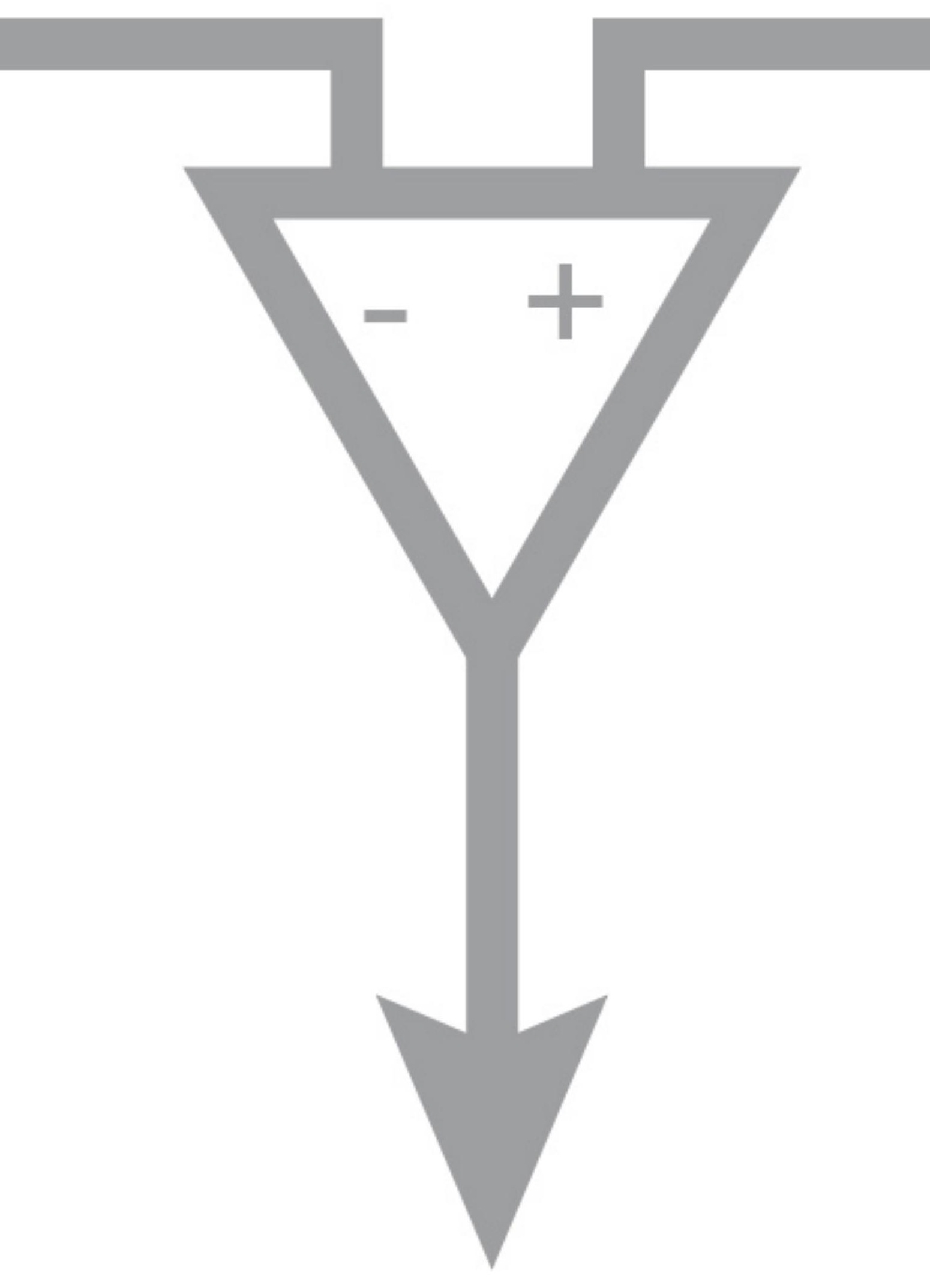

Sound \& reward

Close-up

camera

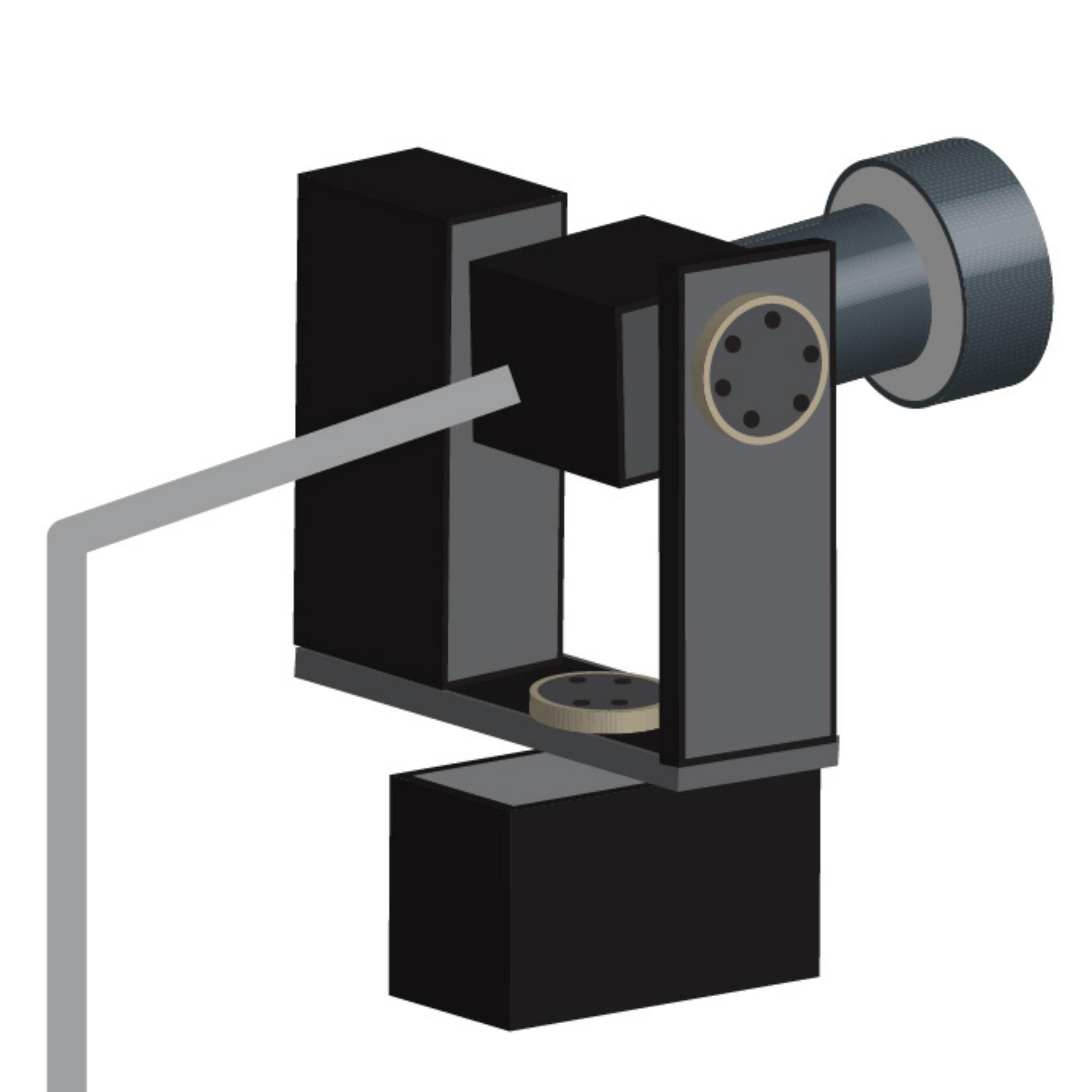

ת
Annotated live image

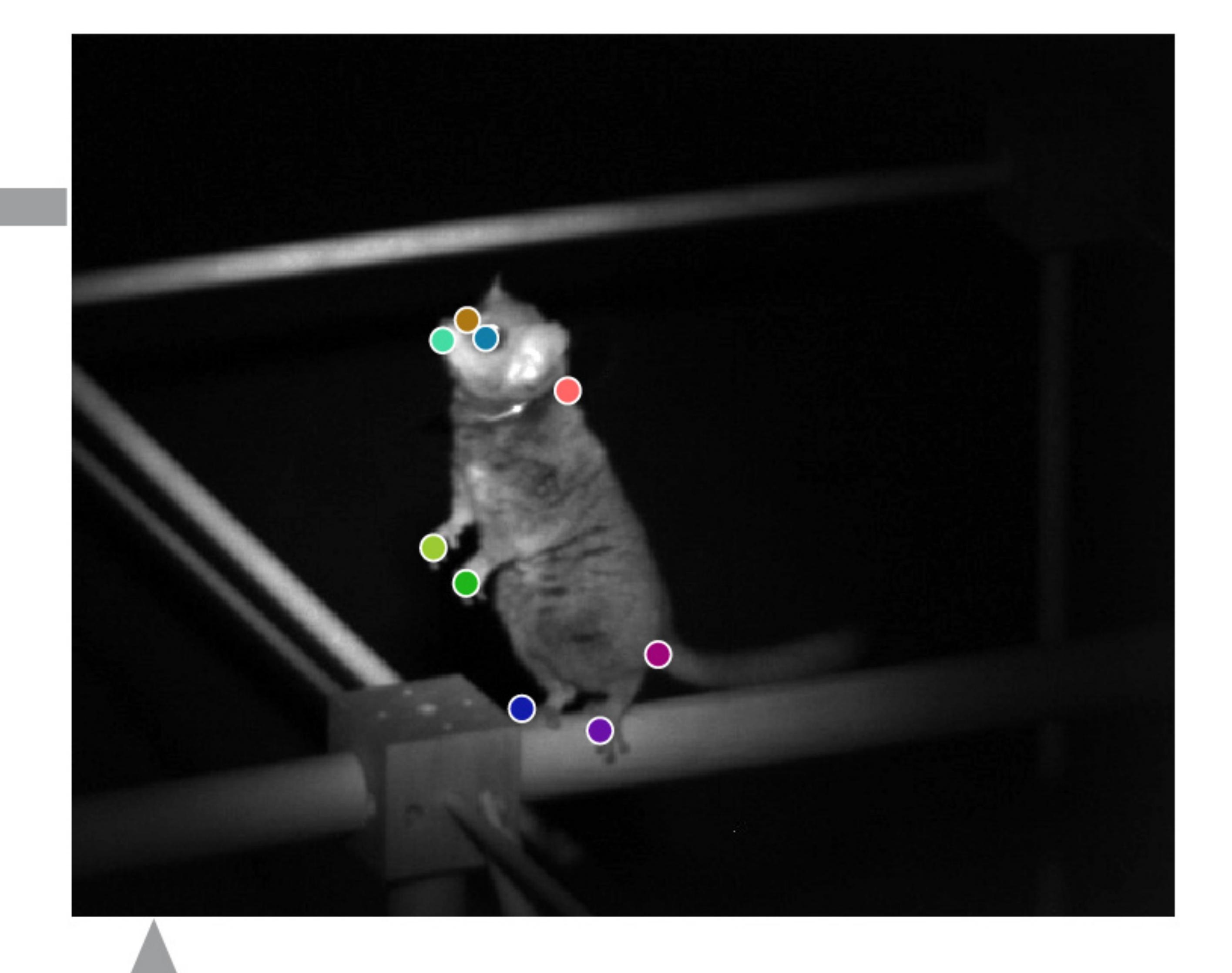

Deep learningbased annotation
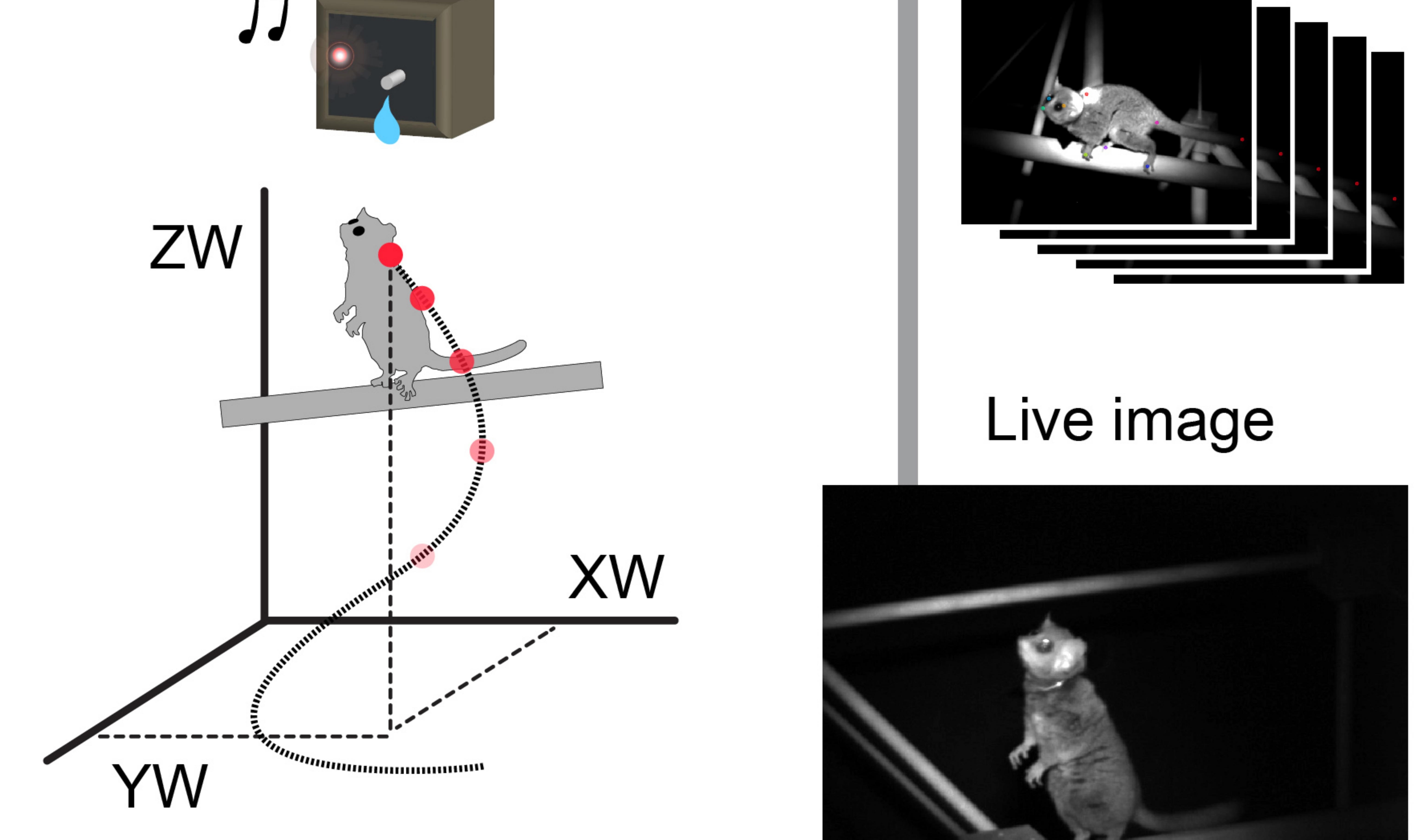

Live image b

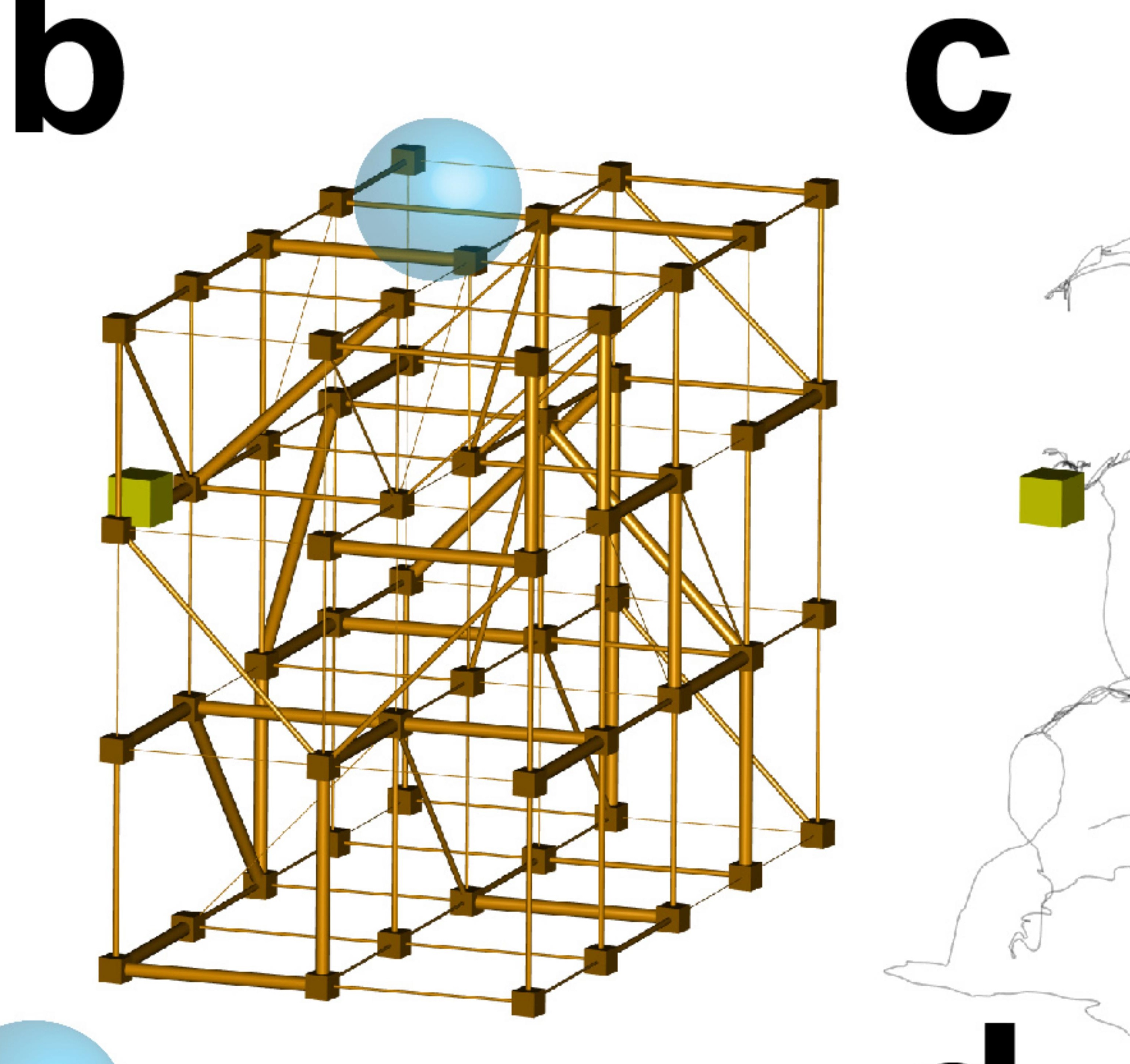

Rearing location d Beginning

RECO-box

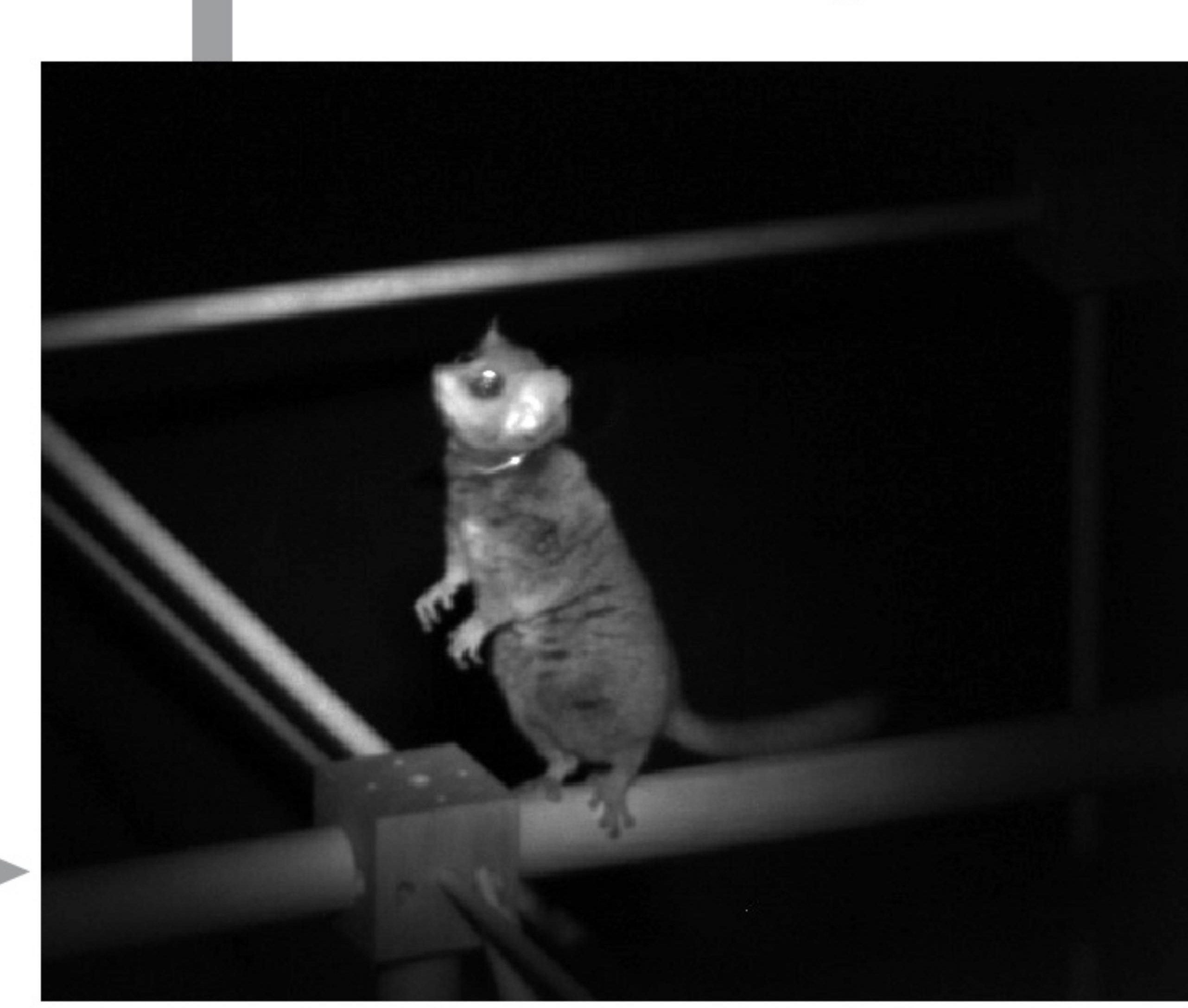

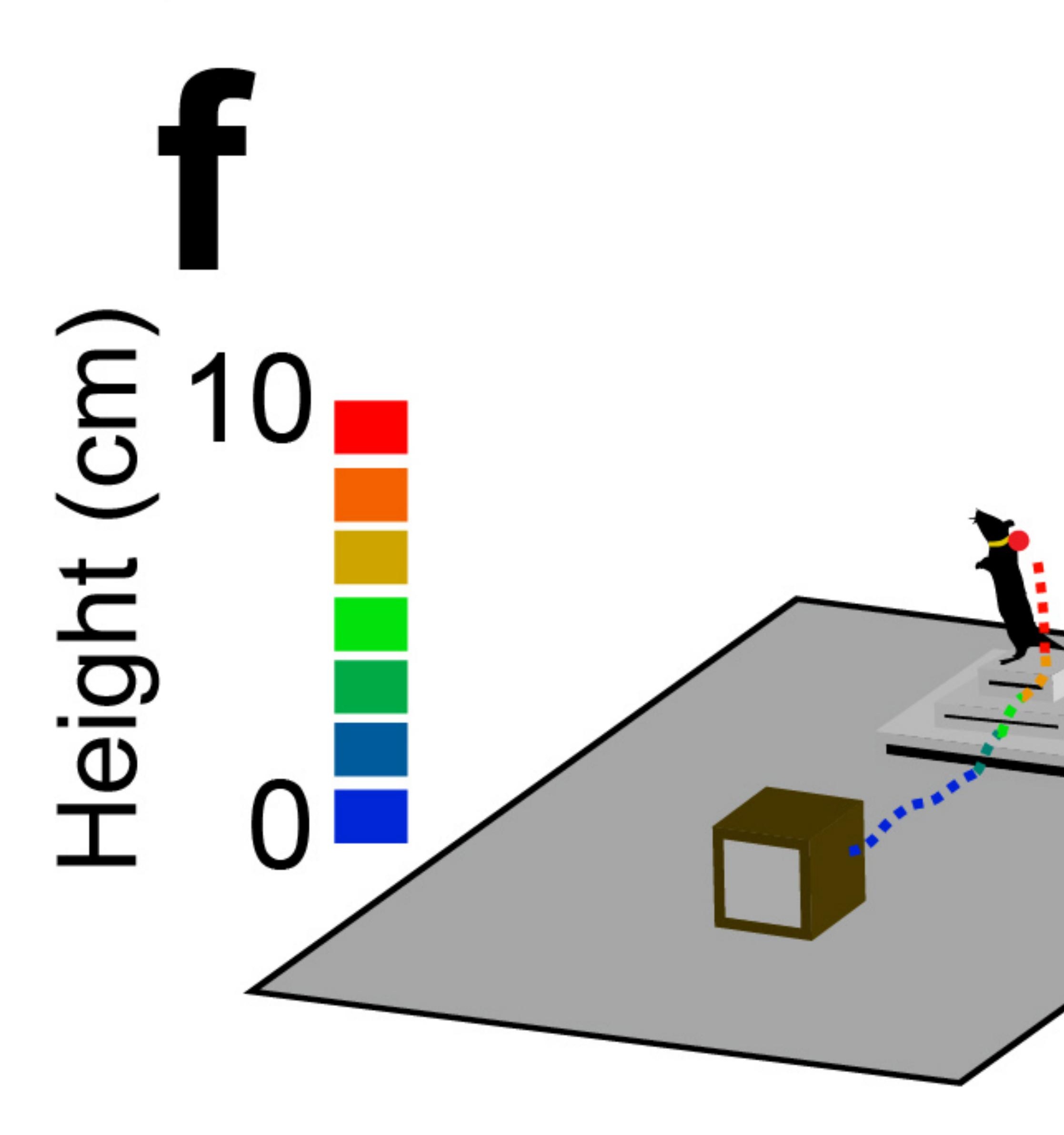

5
Rearing location

RECO-box

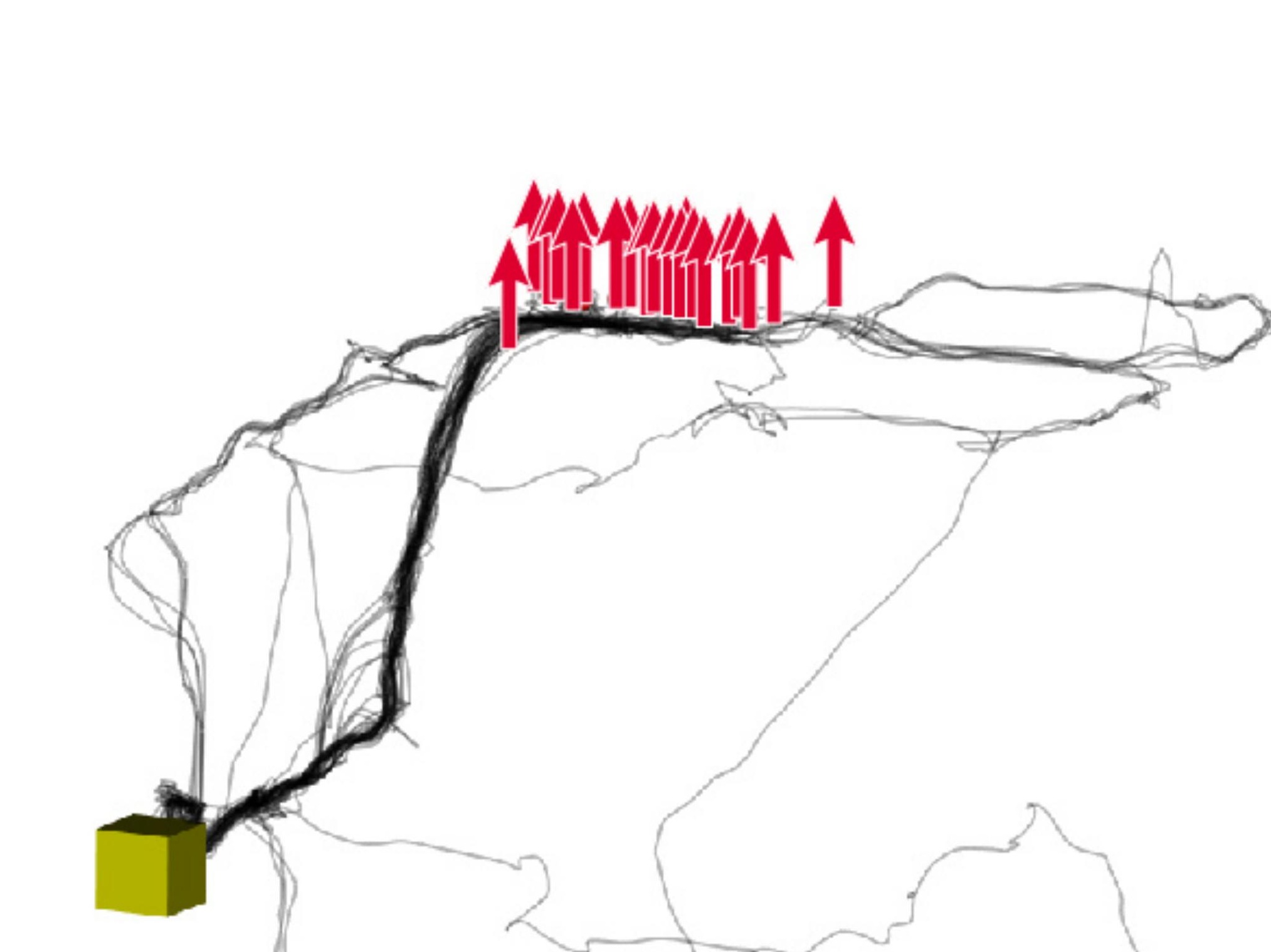

$\sqrt{2}$

Middle

g

h Beginning

Middle

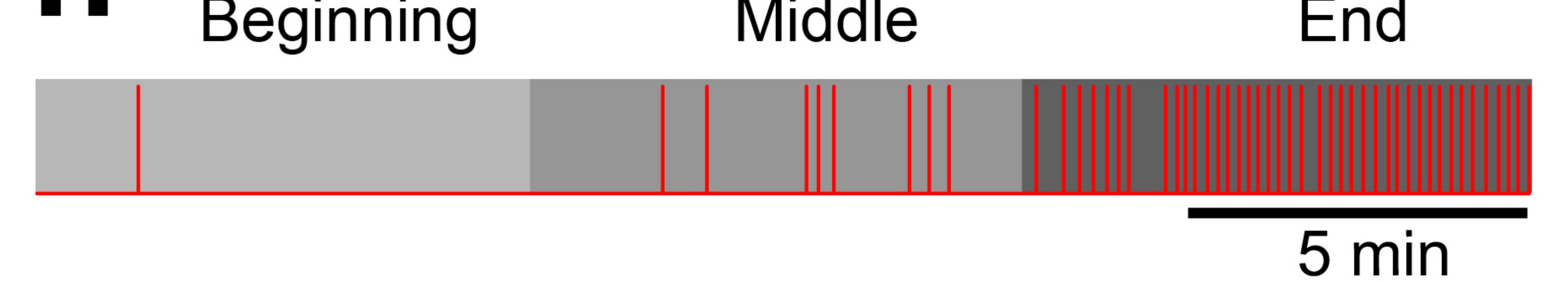

个Rearing $z$

End

End
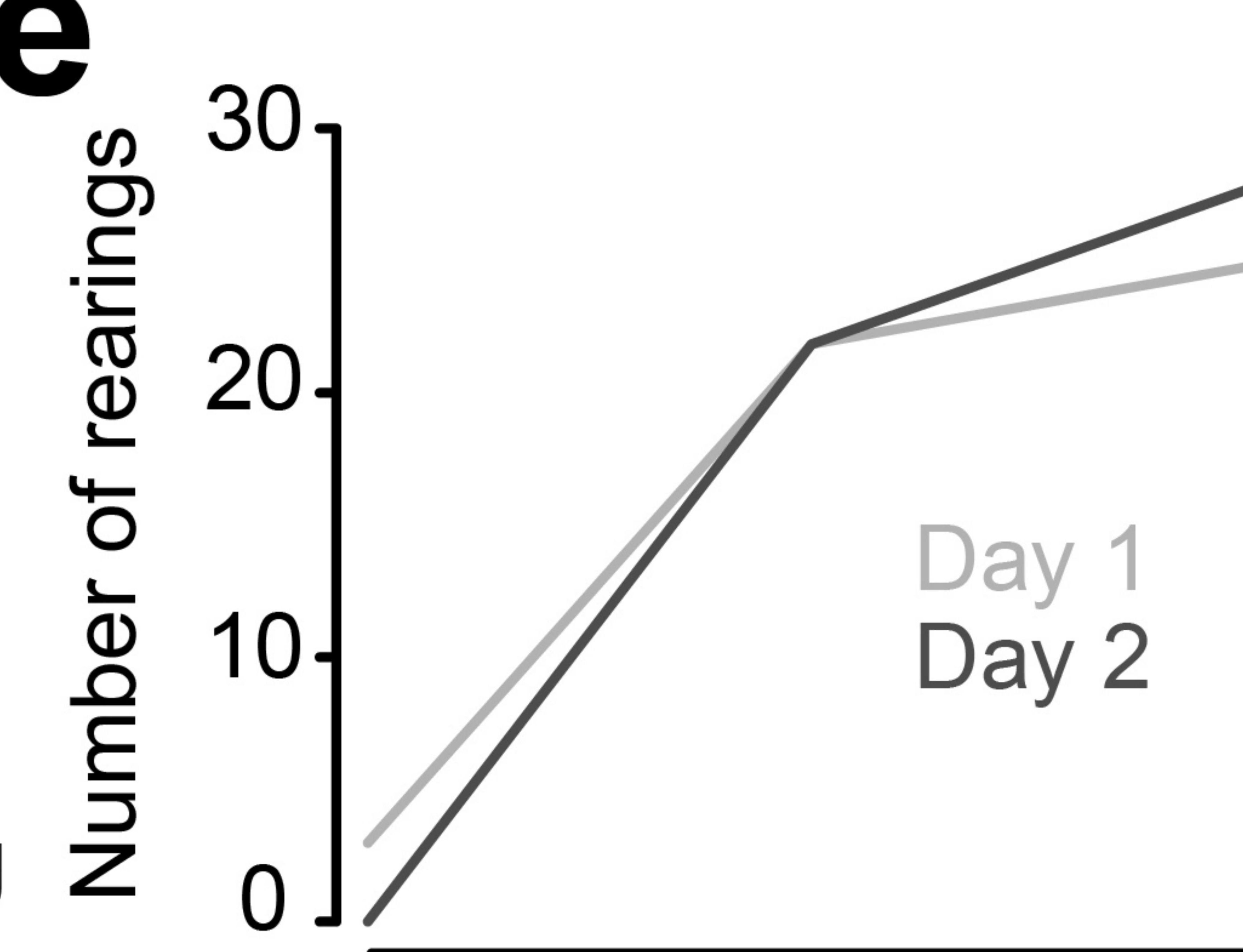

Beginning Middle

End
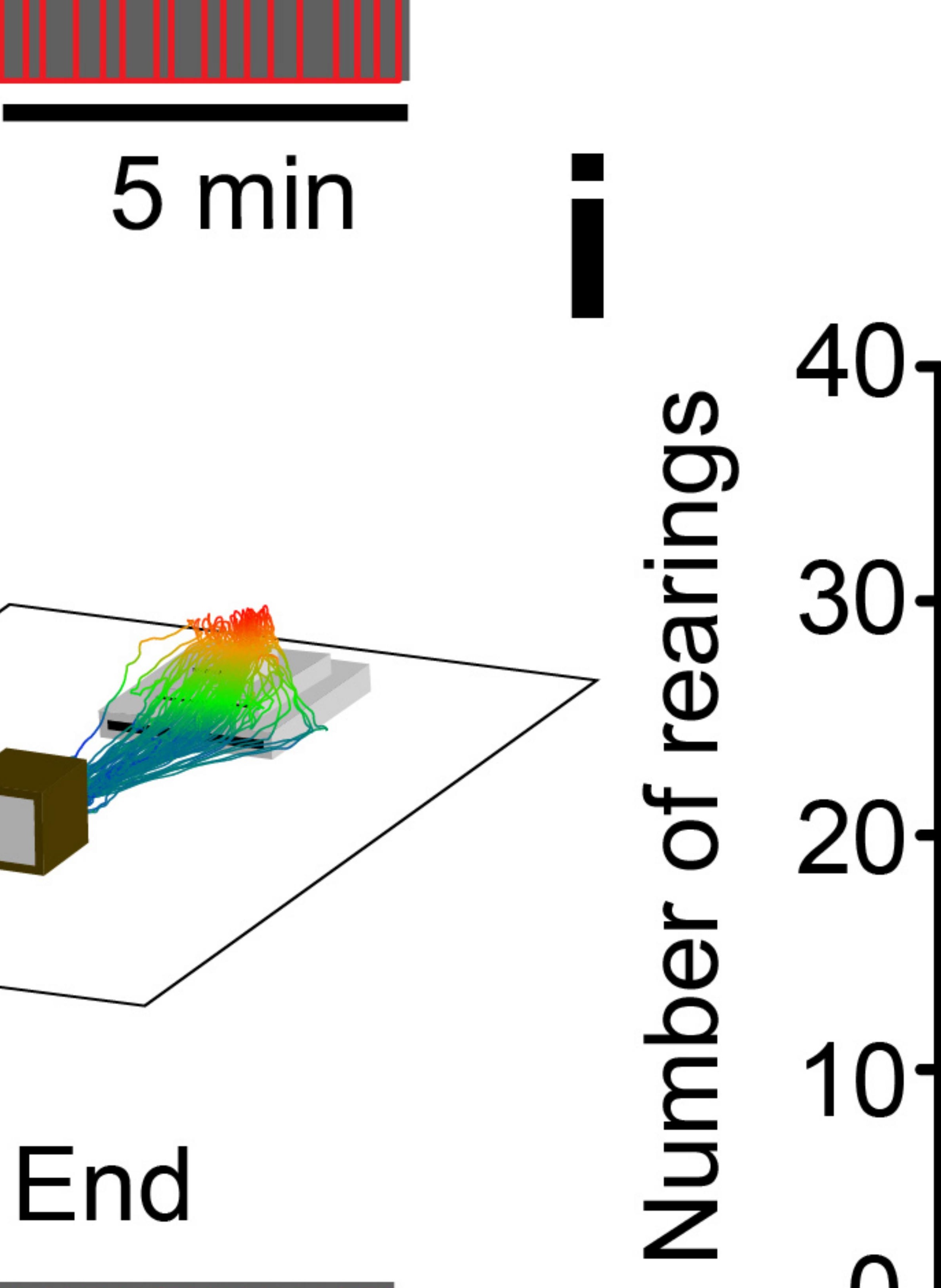

Beginning Middle

End 

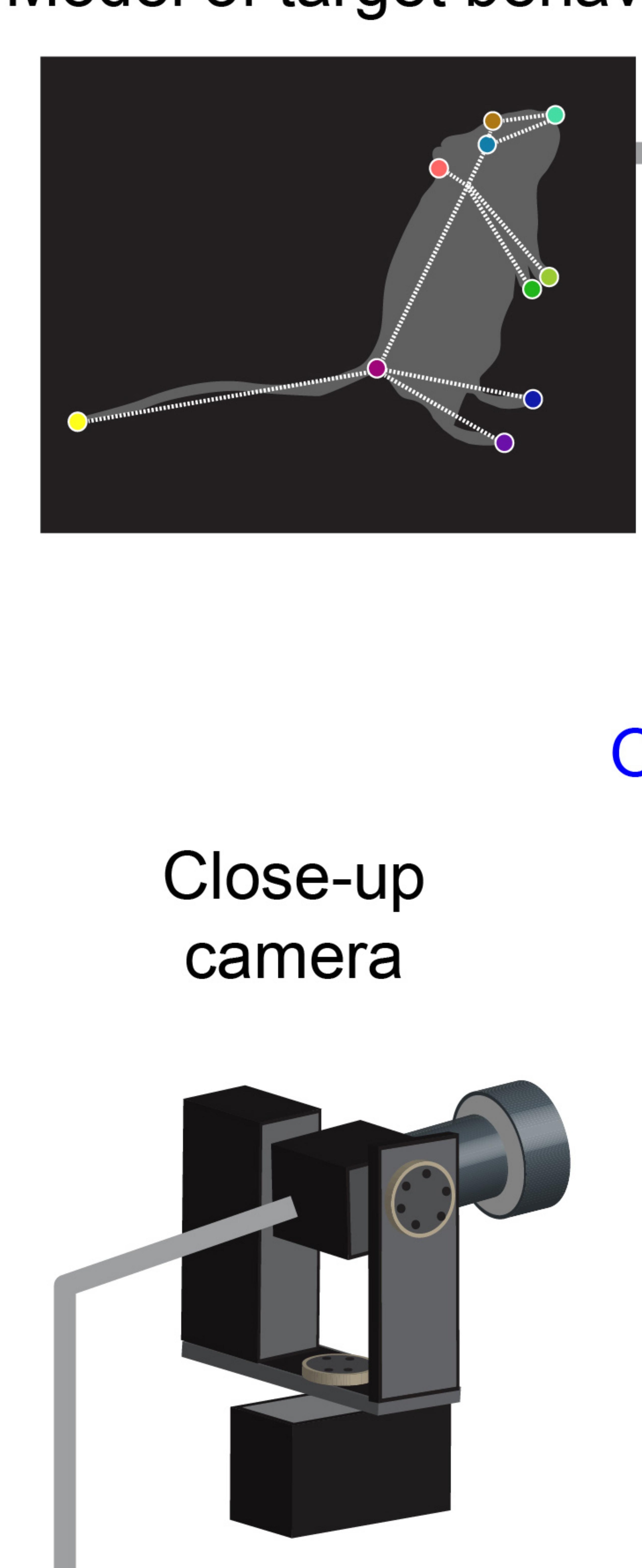

Behavioral detection

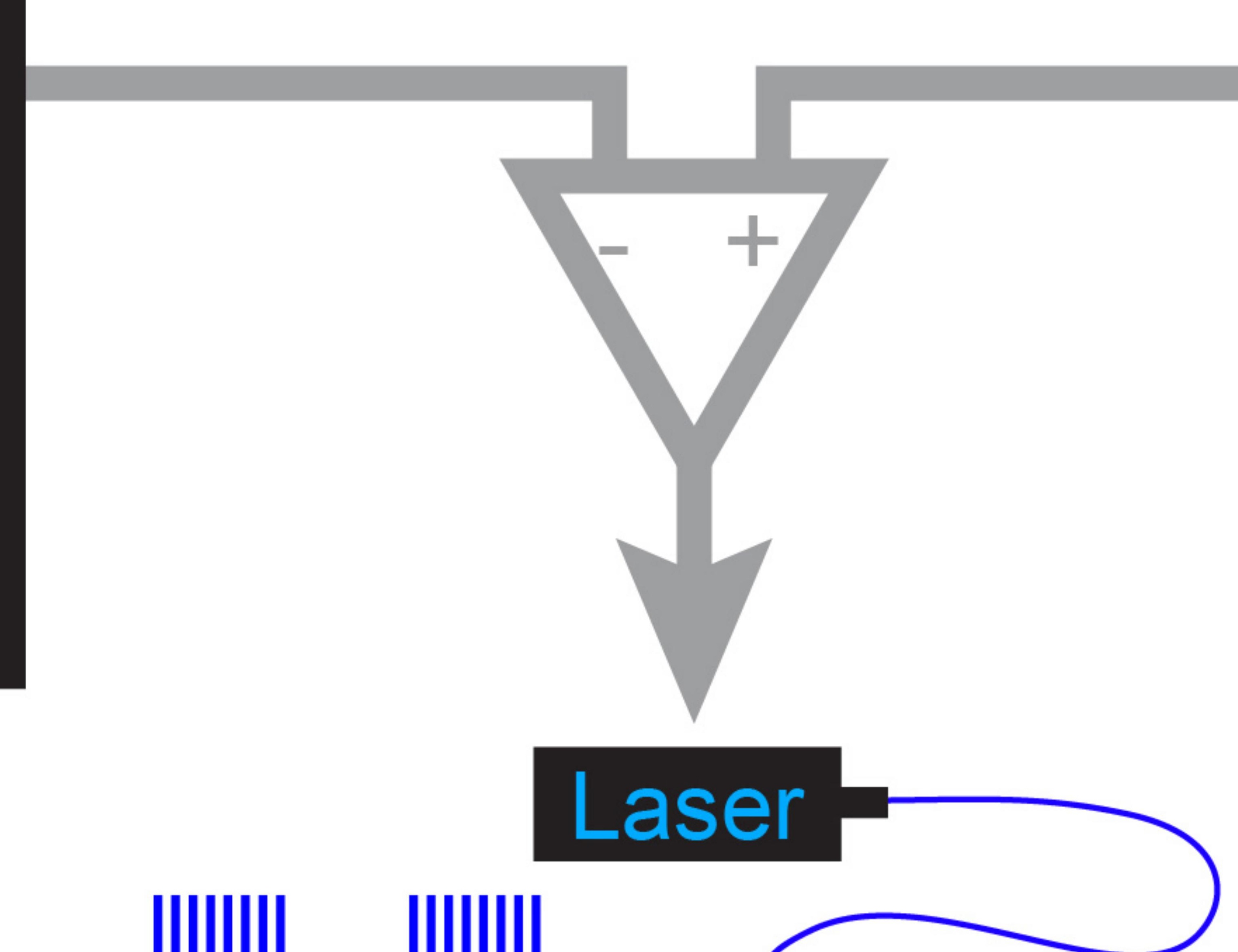

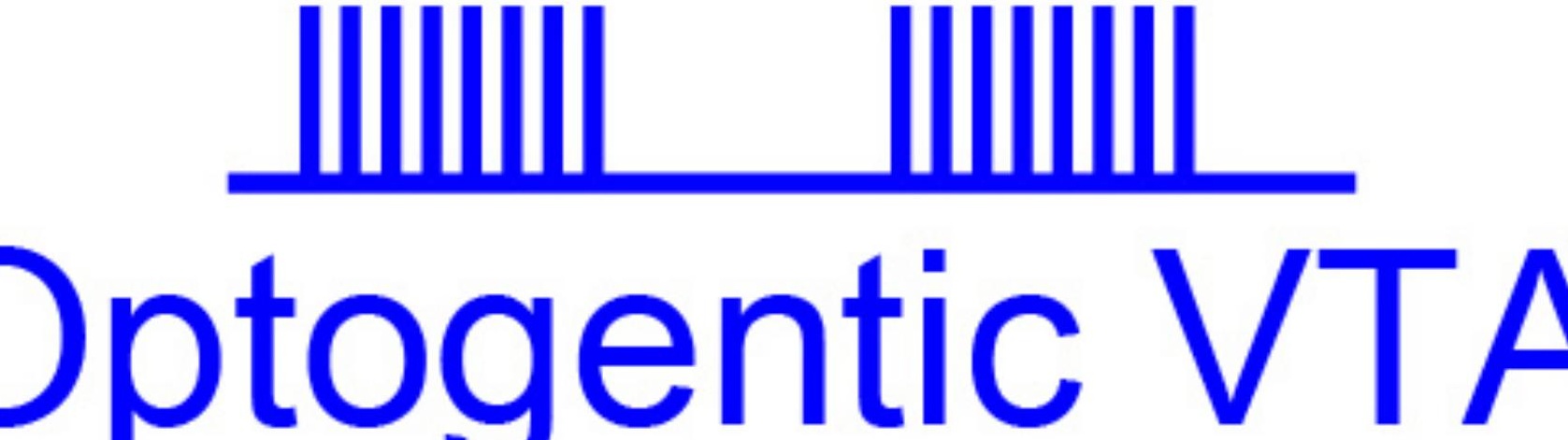

stimulation

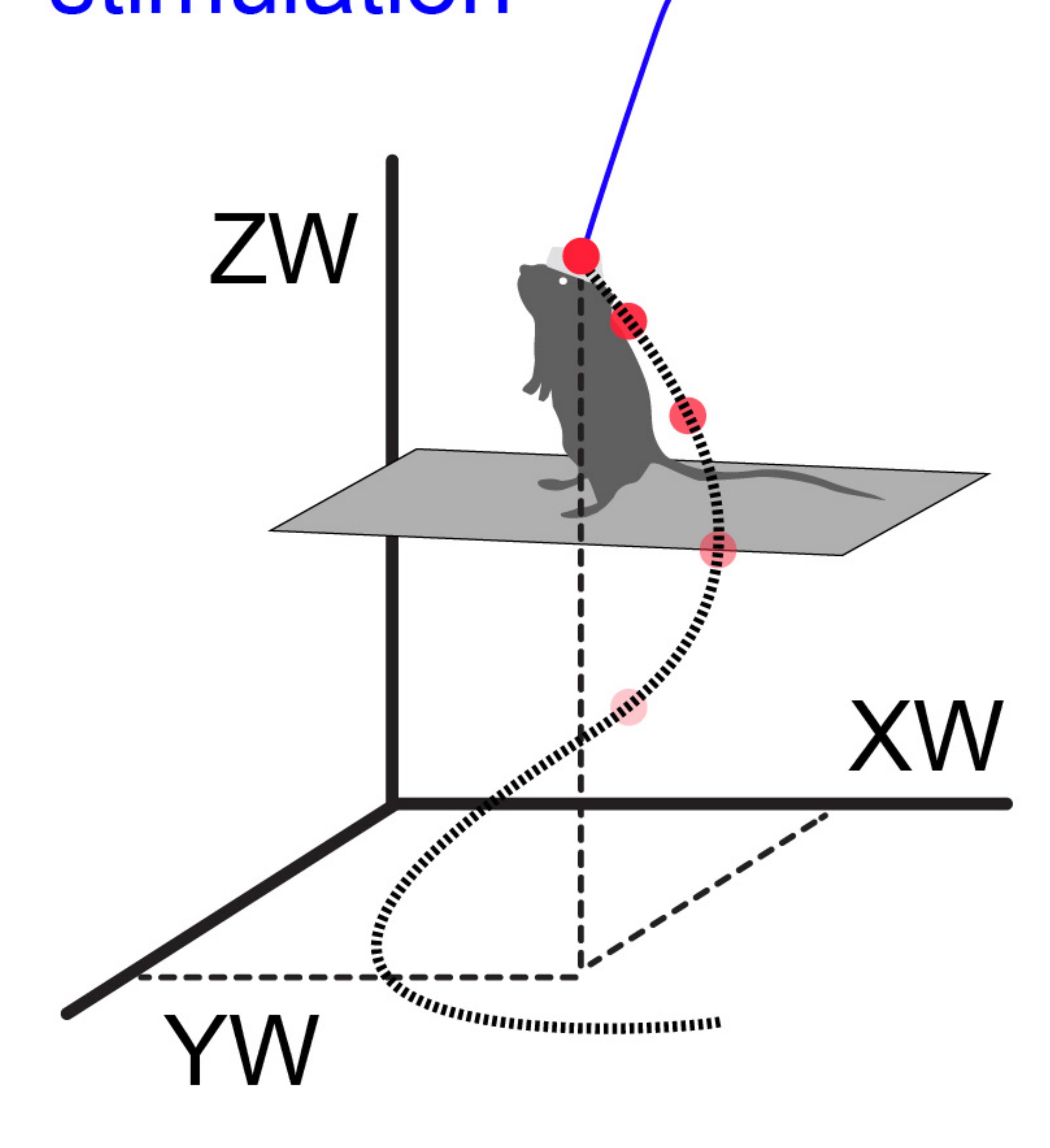

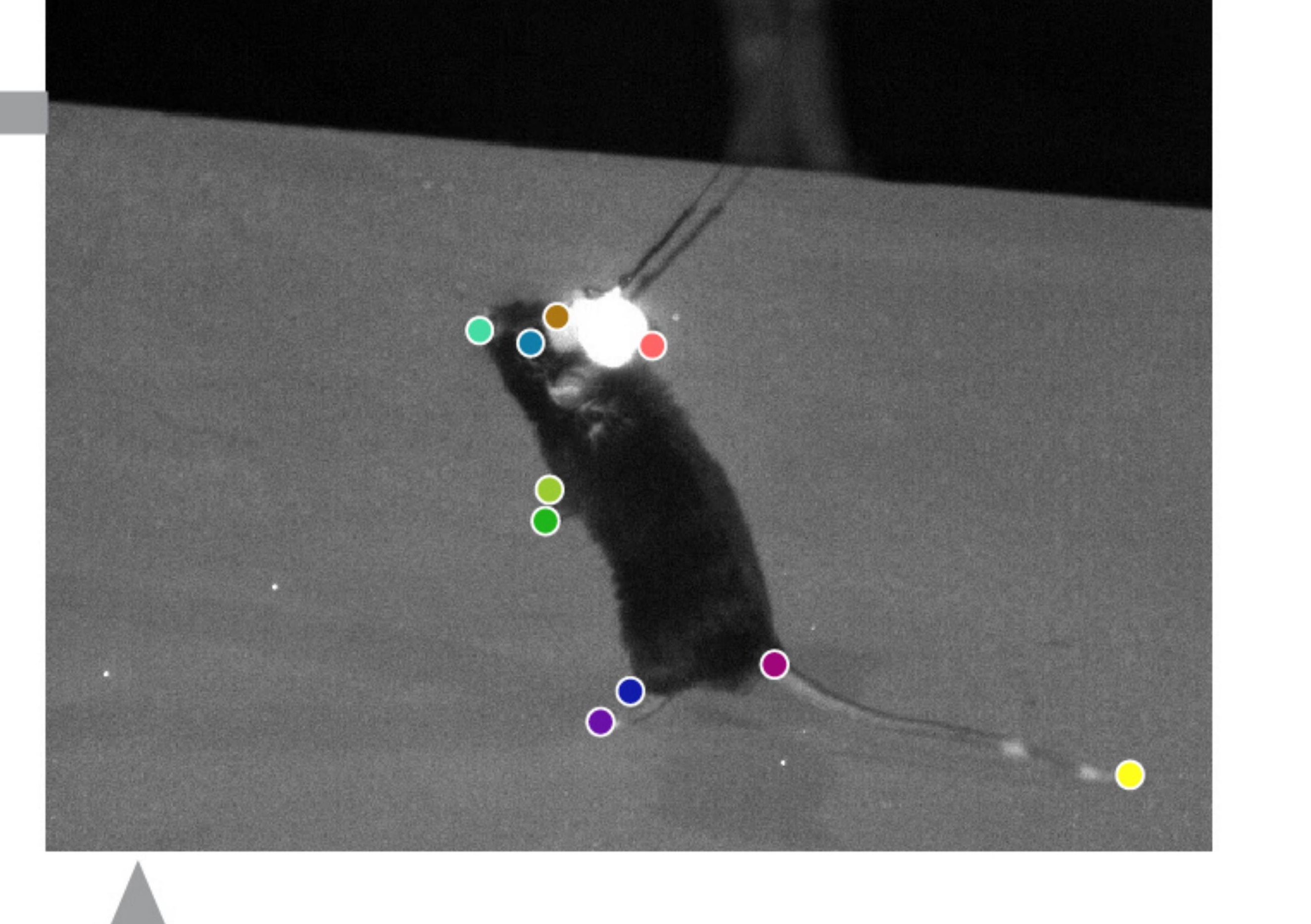

Deep learningbased annotation

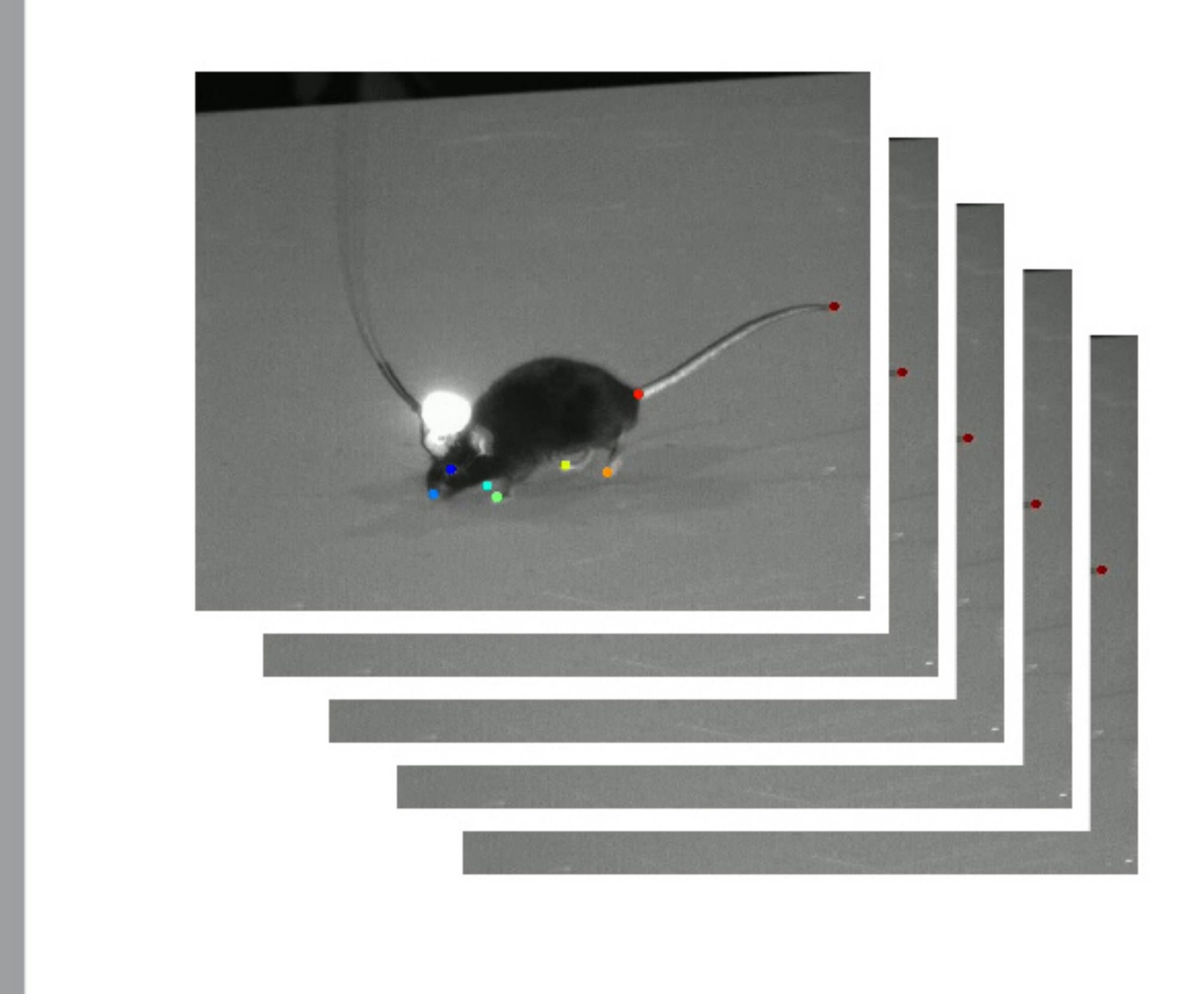

Live image

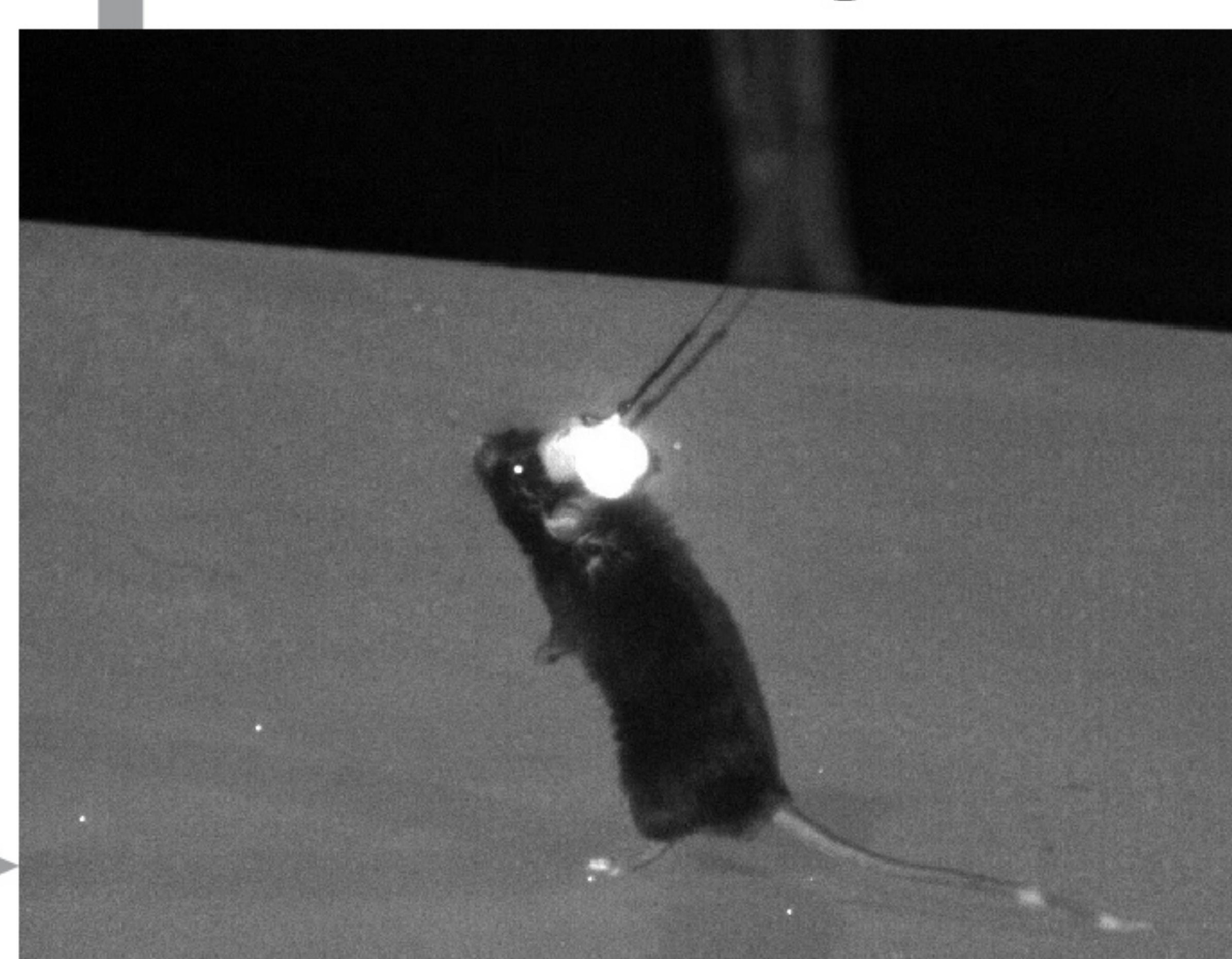

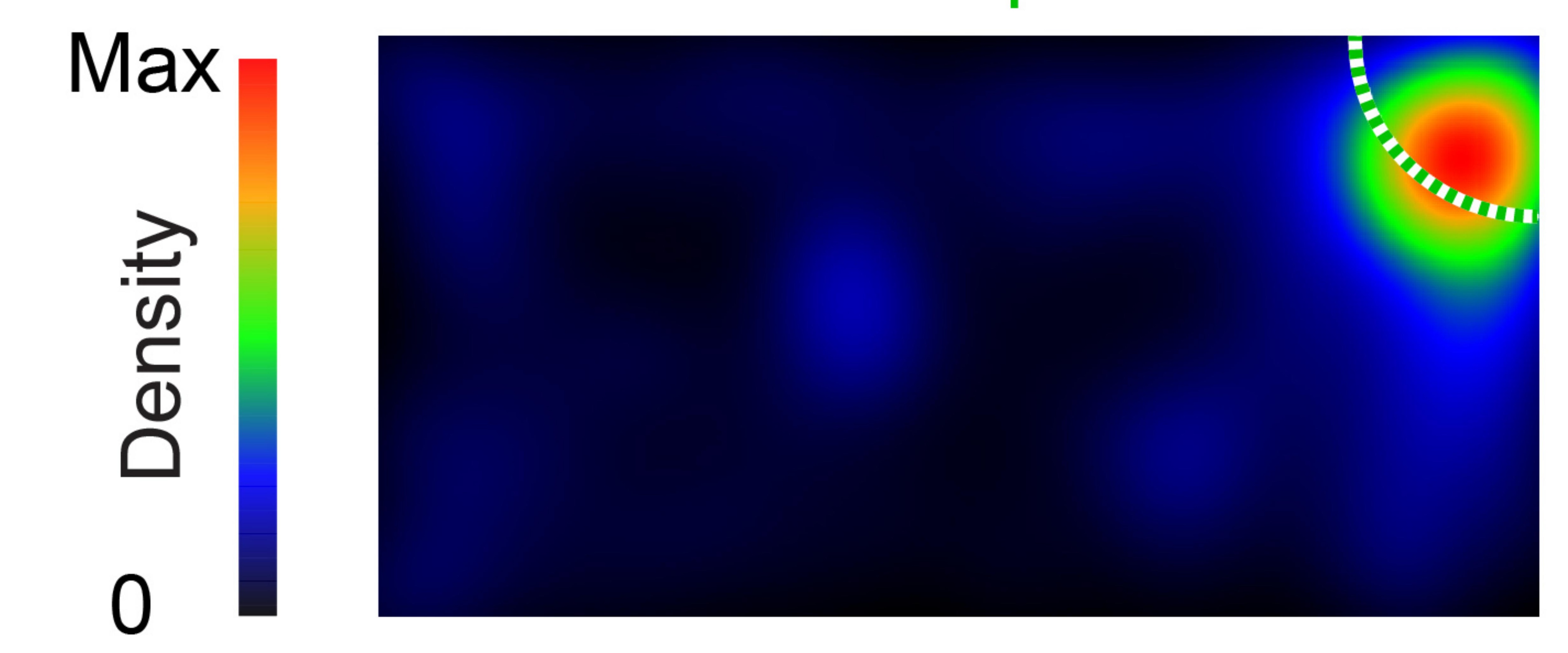

C

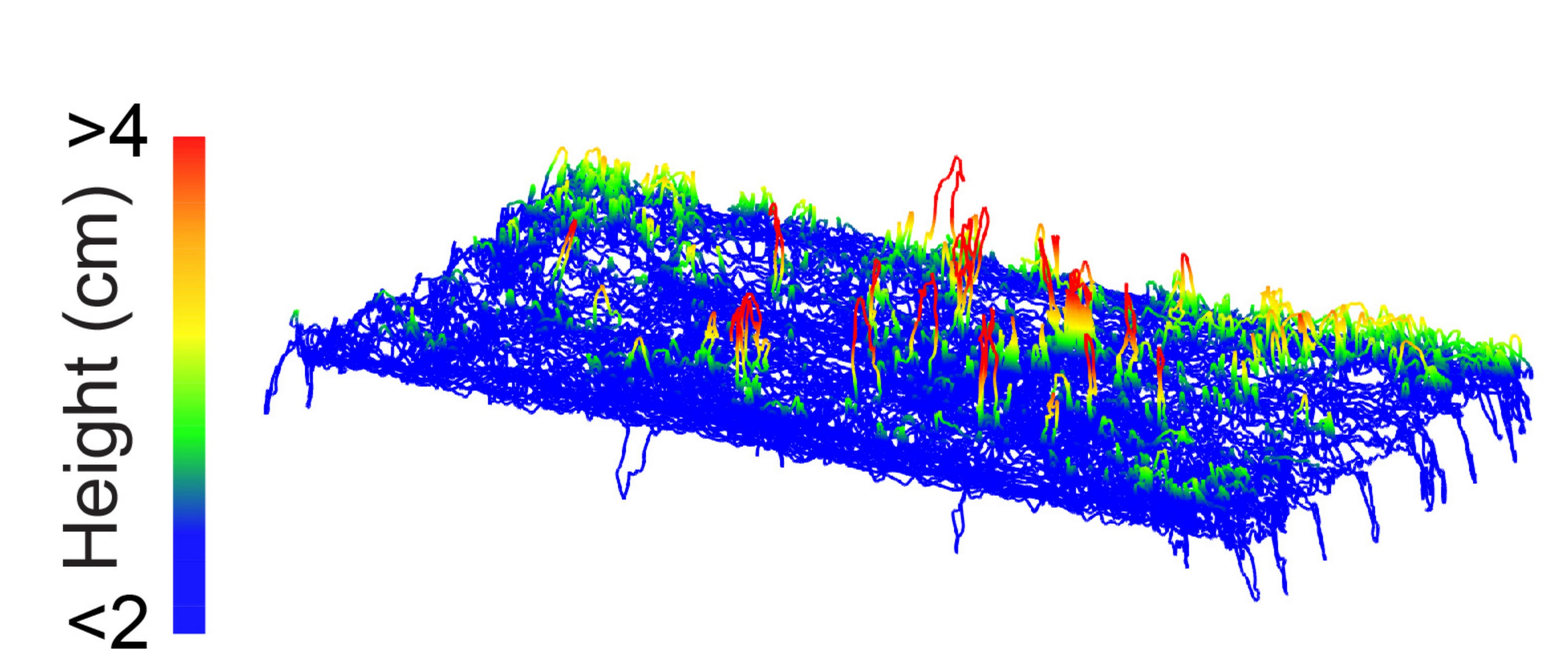

Beginning
Conditioned place: P2
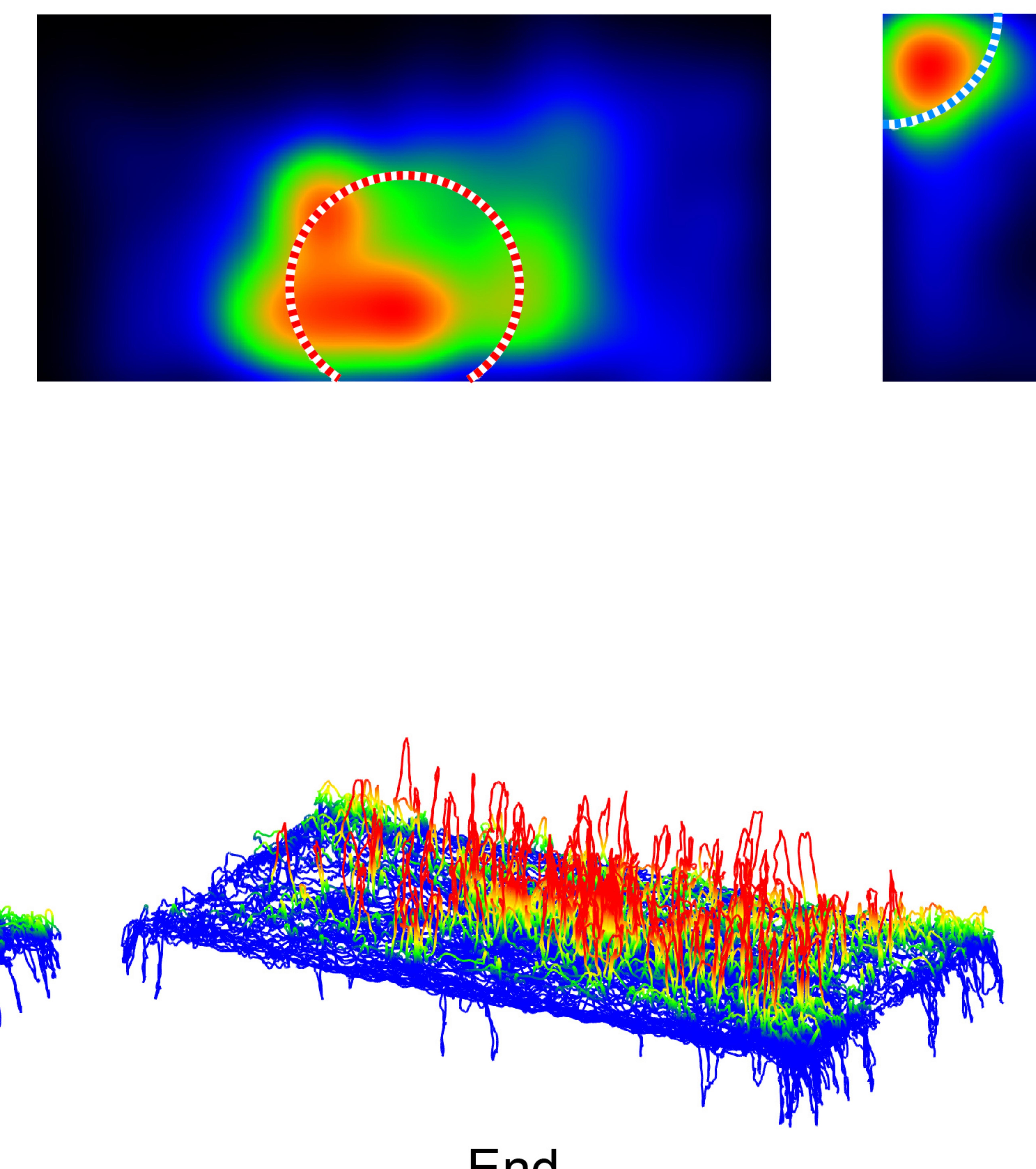

End

\section{d}

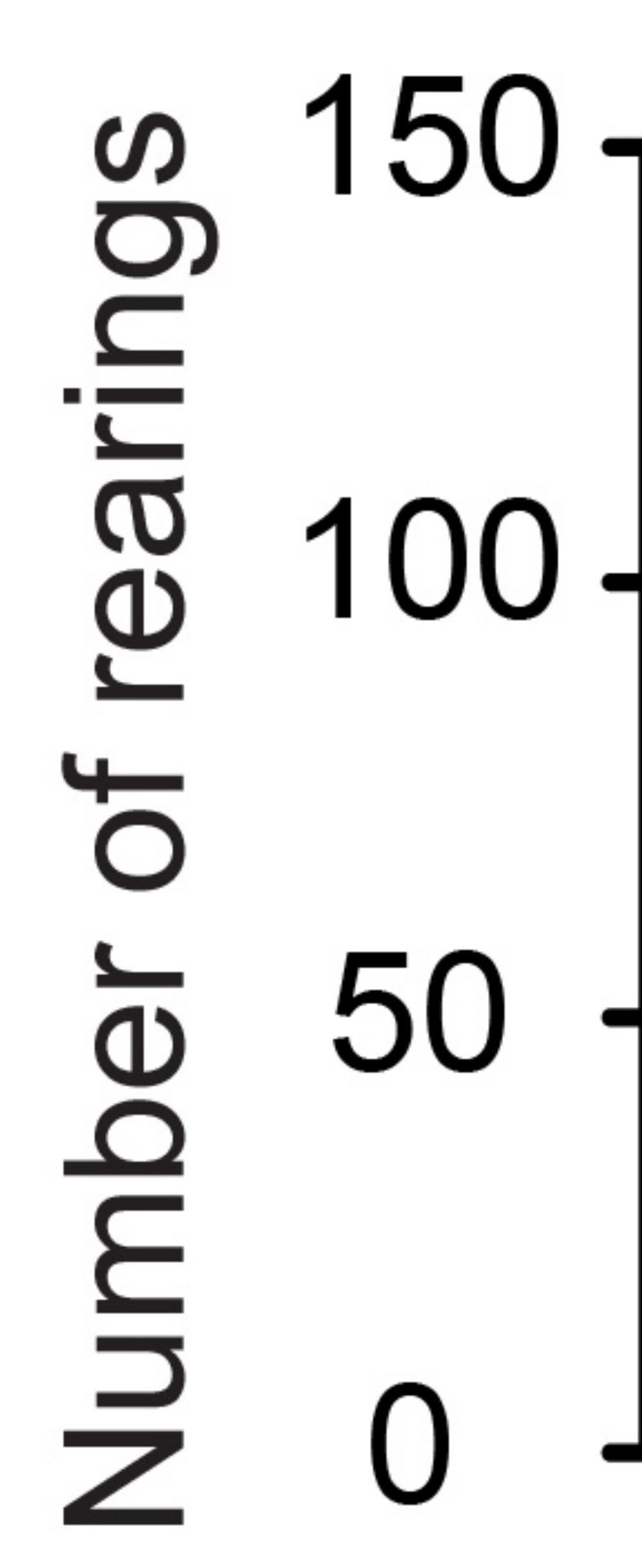

Beginning Middle
Conditioned place: P3
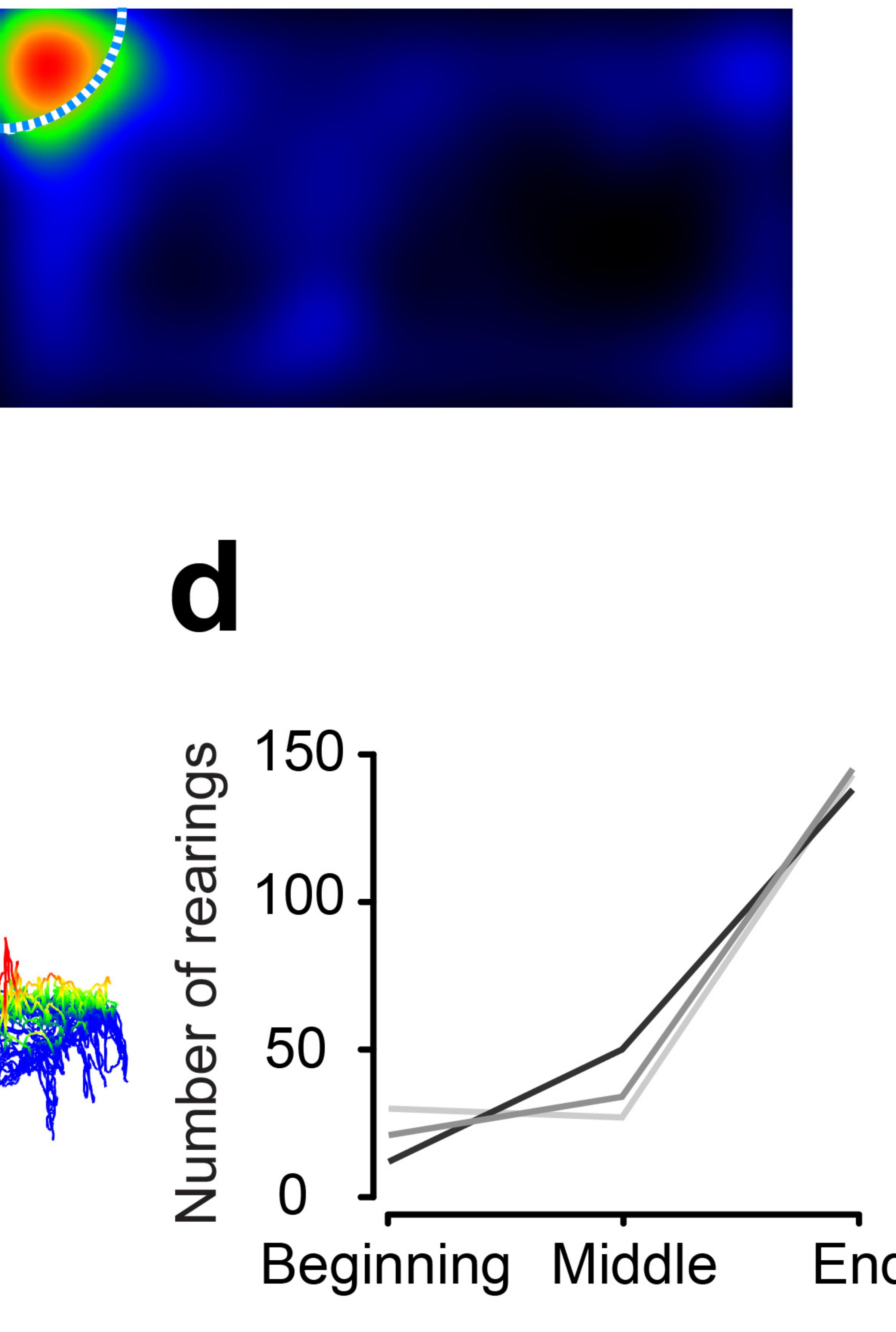
